University of Tennessee Health Science Center

UTHSC Digital Commons

\title{
The Development and Application of a Custom Robotic Biomechanical Testing Platform Employing Real-time Load- control to Compare Spinal Biomechanical Testing Protocols: Pure Moment, Ideal Follower Load, and a Novel Trunk Weight Protocol
}

Charles Ramsey Bennett

University of Tennessee Health Science Center

Follow this and additional works at: https://dc.uthsc.edu/dissertations

Part of the Equipment and Supplies Commons, and the Investigative Techniques Commons

\section{Recommended Citation}

Bennett, Charles Ramsey, "The Development and Application of a Custom Robotic Biomechanical Testing Platform Employing Real-time Load-control to Compare Spinal Biomechanical Testing Protocols: Pure Moment, Ideal Follower Load, and a Novel Trunk Weight Protocol" (2013). Theses and Dissertations (ETD). Paper 331. http://dx.doi.org/10.21007/etd.cghs.2013.0026. 


\title{
The Development and Application of a Custom Robotic Biomechanical Testing Platform Employing Real-time Load-control to Compare Spinal Biomechanical Testing Protocols: Pure Moment, Ideal Follower Load, and a Novel Trunk Weight Protocol
}

\begin{abstract}
The human lumbar spine has been the subject of biomechanical study for many decades owing to the numerous medical cases resulting in the development of various corrective surgical procedures and medical devices intended to relieve patient discomfort. Spinal biomechanics is a broad field containing but not limited to the in vitro study of cadaveric tissue utilizing testing platforms used to apply motion- or load-profiles to tissue in the investigation of the various kinetic or kinematic responses, respectively. The particular arena field of this research concerns the field of robotics as it applies to testing platforms and how they are applied to lumbar spine biomechanical testing.
\end{abstract}

The in vivo spine is subject to six degrees of freedom (DOF) of motion as a consequence of the applied loads of surrounding musculature which apply component loads in 6 DOF. However current in vitro standard protocols apply isolated loads primarily in the anatomical planes. Although the primary goal of in vitro testing may not be the simulation of in vivo circumstances, the accurate recreation of the in vivo loading environment would reveal much regarding the passive biomechanics of the spine. To accomplish such a goal, it would be ideal to utilize a platform capable of providing 6 DOF of controlled mobility as well as capable of apply controlled load in those 6 DOF.

The Musculoskeletal Research Laboratory has developed such a system. The system's load-control capabilities were validated by simulating two standard biomechanical protocols, the pure moment and the ideal follower load on 6 L4-L5 single motion segment units. The robotic performance of the system was evaluated by measuring the tracking errors during testing, or the difference between experimental forces being applied and the forces commanded by the custom motion programs executed during protocol simulation. The biomechanical data that was recorded and compared to the literature for validation was rotational range of motion in the sagittal plane and anatomical point translation. Translation data proved to be difficult to compare effectively to the literature due to the sparseness of comparable numbers. There was also interest in the platform's ability to control protocols. To test this hypothesis, three different biomechanical protocols were simulated and there biomechanical results were compared: pure moment, ideal follower load, and trunk weight.

The system provided stable, good load-control in during combined motions involving all 6 DOF. The tracking errors observed were low compared to other published robotic biomechanical platforms. The mean combined flexion-extension rotational range of motion in the sagittal plane for the pure moment protocol, the ideal follower load, and the trunk weight protocols were $8.2^{\circ}\left( \pm 2.5^{\circ}\right), 7.6^{\circ}\left( \pm 2.9^{\circ}\right)$, and $7.4^{\circ}\left( \pm 2.8^{\circ}\right)$, respectively. There were statistically significant differences in the absolute translational data across the protocols but when comparing relative changes due to flexion and extension only, there are no significant differences across protocols.

In conclusion to this research the platform developed and validated in the current study adequately provides the capabilities of 6 DOF coordinated motion and 5 DOF coordinated load-control. It is sufficient to simulate the standard spine biomechanical test protocols of pure moment and ideal follower load on single segments. It is also a good tool for comparing the effects of particular protocols on the passive biomechanics of human cadaveric tissue. To the author's knowledge, this is the first publication of a fully robotic system adequately controlling a non-zero dynamic force vector while a bending protocol was being applied to a human spinal segment. This research is limited to the sagittal plane and single lumbar 
spine motion segment units.

Document Type

Thesis

Degree Name

Master of Science (MS)

Program

Biomedical Engineering and Imaging

Research Advisor

Brian P. Kelly, Ph.D.

Keywords

Biomechanics, Cartesian, Load-control, Lumbar Spine, Robotics

Subject Categories

Analytical, Diagnostic and Therapeutic Techniques and Equipment | Equipment and Supplies |

Investigative Techniques | Medicine and Health Sciences

\section{Comments}

Two year embargo expired May 2015 
The Development and Application of a Custom Robotic Biomechanical Testing Platform Employing Real-time Load-control to Compare Spinal Biomechanical Testing Protocols: Pure Moment, Ideal Follower Load, and a Novel Trunk Weight Protocol

\author{
A Thesis \\ Presented for \\ The Graduate Studies Council \\ The University of Tennessee \\ Health Science Center
}

In Partial Fulfillment

Of the Requirements for the Degree

Master of Science

In the Joint Graduate Program in Biomedical Engineering and Imaging

From The University of Tennessee

and

The University of Memphis

By

Charles Ramsey Bennett

May 2013 
Copyright (C) 2013 by Charles Ramsey Bennett.

All rights reserved. 


\section{DEDICATION}

I dedicate this work to my sister, Emily Ann Bennett. 


\section{ACKNOWLEDGEMENTS}

I would like to acknowledge my graduate advisor, Dr. Brian P. Kelly, for his patience and guidance during my time in the Musculoskeletal Research Laboratory, without whose tolerance, calm, and timely words of advice and encouragement, I would have certainly been a severely less pleasant person. I would also like to acknowledge the other members of my advisory committee, Dr. Denis J. DiAngelo and Dr. Richard J. Kasser, for their time, effort, and collaborative efforts in molding my inexperienced mind into what it is today.

I acknowledge the Joint Biomedical Engineering and Imaging program as an exceptional bridging of two high class institutes which boast a satisfactory graduate program. It is my regret to have had limited exposure to the staff at the University of Memphis.

I express my thanks by acknowledging my laboratory peers for providing moral support, participating in valuable brainstorming and consultation meetings, and for giving me company and sanity during the few hours not spent working. Their names are Hunter Smith, John Simmons, Kelly Salb, Tom Stewart, Andrew Herndon, Quincy Beasley, Erik Woodard, and Casey Hebert.

I would like to acknowledge those who suffered through many brainstorming conversations outside the laboratory environment that involved ideation and napkin drawings: my father, Greg Bennett, a fellow engineer, Frank Welsh, a former master's student, Brian Morrow, and the go-to machinist in the UTHSC machine shop, Michael Nguyen.

Finally I would like to acknowledge my wife, Rachel Bennett, who trusted me with her life, followed me to Memphis, and has been supportive since the beginning by showing unwavering devotion and having only the highest opinion of my limited capabilities. Without this woman, I would be far less happy and indescribably more hungry. 


\begin{abstract}
The human lumbar spine has been the subject of biomechanical study for many decades owing to the numerous medical cases resulting in the development of various corrective surgical procedures and medical devices intended to relieve patient discomfort. Spinal biomechanics is a broad field containing but not limited to the in vitro study of cadaveric tissue utilizing testing platforms used to apply motion- or load-profiles to tissue in the investigation of the various kinetic or kinematic responses, respectively. The particular arena field of this research concerns the field of robotics as it applies to testing platforms and how they are applied to lumbar spine biomechanical testing.
\end{abstract}

The in vivo spine is subject to six degrees of freedom (DOF) of motion as a consequence of the applied loads of surrounding musculature which apply component loads in 6 DOF. However current in vitro standard protocols apply isolated loads primarily in the anatomical planes. Although the primary goal of in vitro testing may not be the simulation of in vivo circumstances, the accurate recreation of the in vivo loading environment would reveal much regarding the passive biomechanics of the spine. To accomplish such a goal, it would be ideal to utilize a platform capable of providing 6 DOF of controlled mobility as well as capable of apply controlled load in those 6 DOF.

The Musculoskeletal Research Laboratory has developed such a system. The system's load-control capabilities were validated by simulating two standard biomechanical protocols, the pure moment and the ideal follower load on 6 L4-L5 single motion segment units. The robotic performance of the system was evaluated by measuring the tracking errors during testing, or the difference between experimental forces being applied and the forces commanded by the custom motion programs executed during protocol simulation. The biomechanical data that were recorded and compared to the literature for validation were rotational range of motion in the sagittal plane and anatomical point translation. Translation data proved to be difficult to compare effectively to the literature due to the sparseness of comparable numbers. There was also interest in the platform's ability to control protocols. To test this hypothesis, three different biomechanical protocols were simulated and there biomechanical results were compared: pure moment, ideal follower load, and trunk weight.

The system provided stable, good load-control during combined motions involving all $6 \mathrm{DOF}$. The tracking errors observed were low compared to other published robotic biomechanical platforms. The mean combined flexion-extension rotational range of motion in the sagittal plane for the pure moment protocol, the ideal follower load, and the trunk weight protocols were $8.2^{\circ}\left( \pm 2.5^{\circ}\right), 7.6^{\circ}\left( \pm 2.9^{\circ}\right)$, and $7.4^{\circ}\left( \pm 2.8^{\circ}\right)$, respectively. There were statistically significant differences in the absolute translational data across the protocols but when comparing relative changes due to flexion and extension only, there were no significant differences across protocols.

In conclusion to this research the custom robotic biomechanical testing platform developed and validated in the current study adequately provides the capabilities of 6 
DOF coordinated motion and 5 DOF coordinated load-control. It was sufficient to simulate the standard spine biomechanical test protocols of pure moment and ideal follower load on single segments. It was also a good tool for comparing the effects of particular protocols on the passive biomechanics of human cadaveric tissue. To the author's knowledge, this is the first publication of a fully robotic system adequately controlling a non-zero dynamic force vector while a bending protocol was being applied to a human spinal segment. This research was limited to the sagittal plane and single lumbar spine motion segment units. 


\section{TABLE OF CONTENTS}

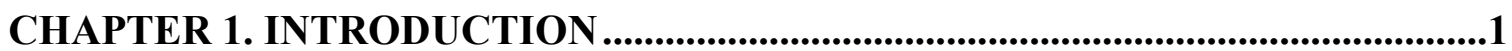

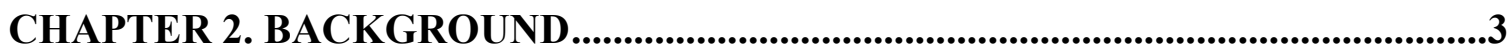

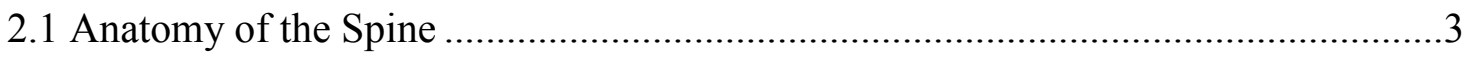

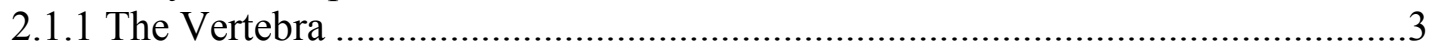

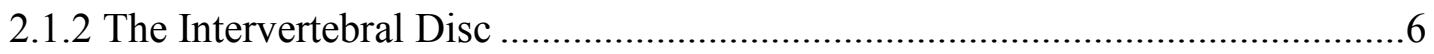

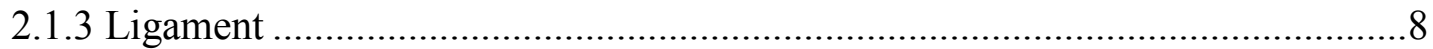

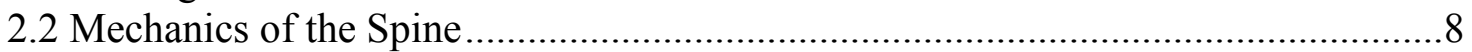

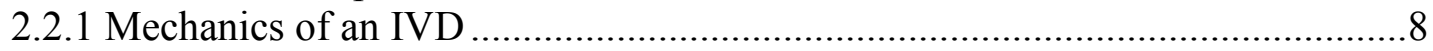

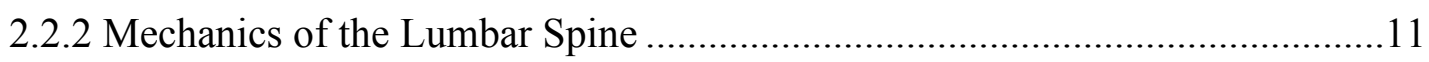

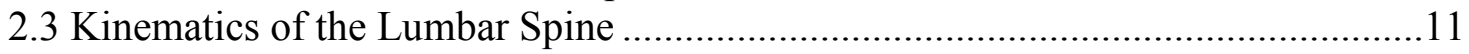

2.3.1 Neutral Zone ………………….........................................................13

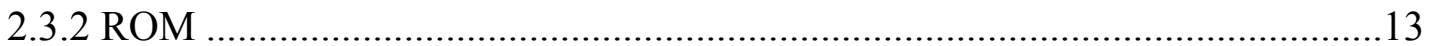

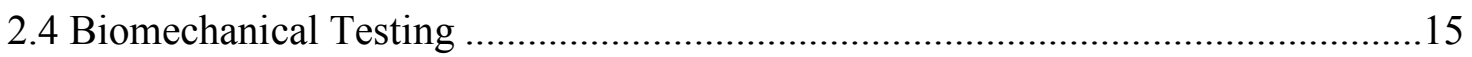

2.4.1 Flexibility versus Stiffness Testing............................................................15

2.4.2 Robotics in Biomechanical Testing …………............................................15

CHAPTER 3. ROBOTIC APPLICATION OF A DYNAMIC RESULTANT FORCE VECTOR USING REAL-TIME LOAD-CONTROL: SIMULATION OF AN IDEAL FOLLOWER LOAD ............................................................18

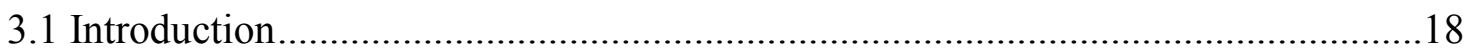

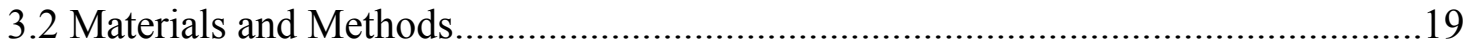

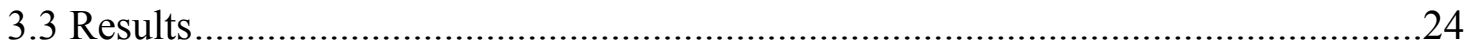

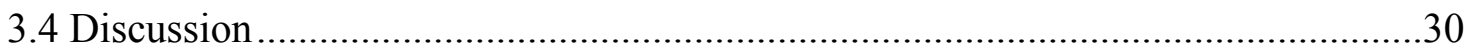

CHAPTER 4. EFFECT OF LOAD ON KINEMATIC BEHAVIOR OF HUMAN LUMBAR SPINE DURING FLEXION-EXTENSION: COMPARISON OF ROBOTICALLY APPLIED PURE MOMENT, IDEAL FOLLOWER LOAD, AND NOVEL TRUNK WEIGHT PROTOCOLS .........................................................32

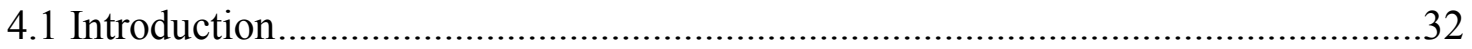

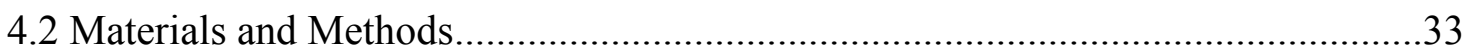

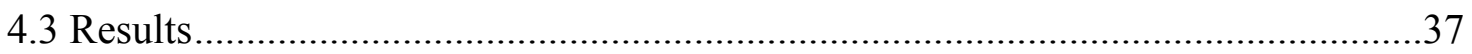

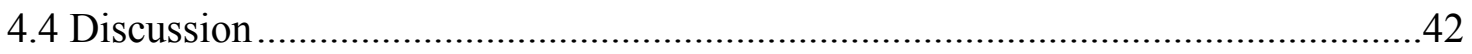

CHAPTER 5. CONCLUSIONS AND LIMITATIONS ............................................49

CHAPTER 6. RECOMMENDATIONS FOR FUTURE WORK ..................................51

LIST OF REFERENCES.......................................................................................................52

APPENDIX A. HARDWARE COMPONENTS AND SPECIFICATIONS ...............58 
APPENDIX B. FORCE/TORQUE CONTROL ALGORITHM...................................59

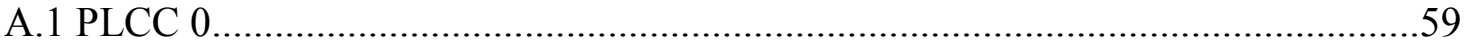

A.2 PLCC 2

APPENDIX C. CUSTOM MOTION PROGRAMS FOR PROTOCOLS ..................67

B.1 Program 14 Pure Moment Pitch Position.................................................................67

B.2 Program 15 Trunk Weight Pitch Position .............................................................

B.3 Program 16 Follower Load Pitch Position ...........................................................75

VITA 


\section{LIST OF TABLES}

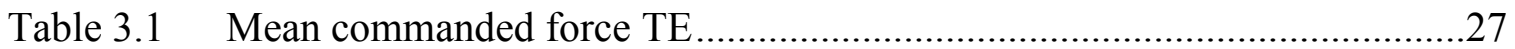

Table 3.2 Mean anatomical point translation in sagittal plane .................................27

Table 4.1 Mean commanded force tracking error ....................................................

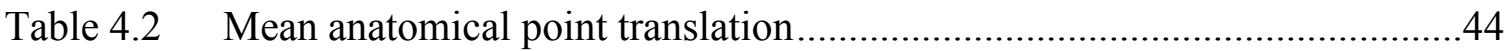

Table A.1 Individual Linear Axis Hardware Specifications.....................................58

Table A.2 Individual Rotary Axis Hardware Specifications ...................................58

Table A.3 JR3 Model 45E15A4-I63-AF Force Moment Sensor Specifications ...........58 


\section{LIST OF FIGURES}

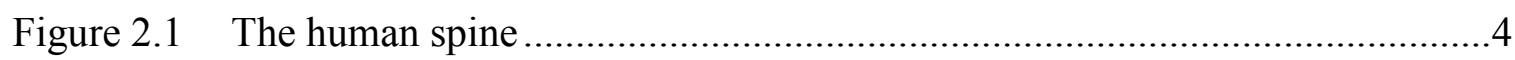

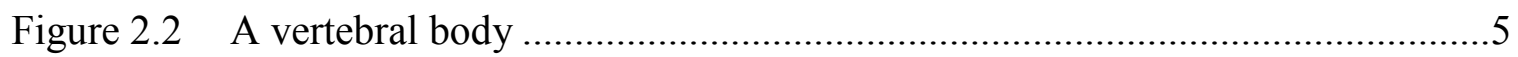

Figure 2.3 The intervertebral disc .....................................................................

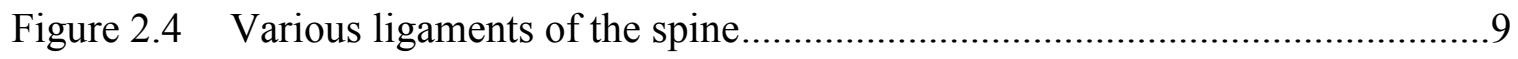

Figure 2.5 Basic IVD mechanical response to loads..................................................10

Figure 2.6 Simplified mechanics of the spine …………........................................12

Figure 2.7 Spinal MSU degrees of freedom................................................................14

Figure 2.8 Custom built robotic biomechanical testing platform...................................17

Figure 3.1 Sagittal plane potting alignment .............................................................20

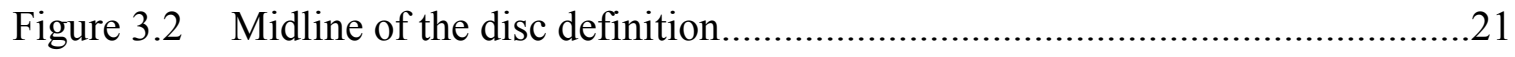

Figure 3.3 Ideal FL application to single MSU ………….......................................22

Figure 3.4 Graphical representation of applied forces ................................................25

Figure 3.5 Graphical representation of applied moments .............................................26

Figure 3.6 Anatomical point translation as result of loading and bending.....................28

Figure 3.7 Rotational motion with respect to applied flexion/extension moments........29

Figure 4.1 Ideal TW application to lumbar MSU ………………………….................36

Figure 4.2 Graphical representation of applied forces and moments during PM

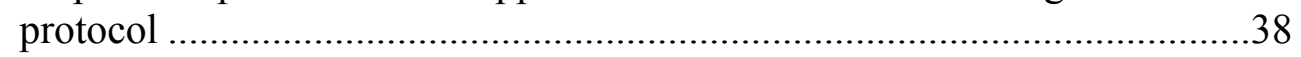

Figure 4.3 Graphical representation of applied forces and moments during FL protocol

Figure 4.4 Graphical representation of applied forces and moments during TW protocol

Figure 4.5 Mean anatomical point translation in different protocols .............................43

Figure 4.6 Mean flexion-extension RROM of different protocols..................................45

Figure 4.7 Single specimen sagittal bend flexibility comparison across protocols........47 


\section{LIST OF ABBREVIATIONS}

$\beta \mathrm{GCS}$

ALL

AP

APT

$\mathrm{CC}$

$\mathrm{COM}$

CRBT

DCS

FDOF

FL

FMS

FMS-CS

FRF

GCS

ICR

IPR

IVD

MRL

MSU

NZ

PLL

PM

ROM

RROM

RTLC

SD

TE.

TW

UTHSC
Beta Global Coordinate System Anterior Longitudinal Ligament Anteroposterior Anatomical Point Translation Craniocaudal Center of Mass .Cartesian Robotic Biomechanical Tester Disc Coordinate System Degrees of Freedom .Follower Load Force-Moment Sensor Force-Moment Sensor Coordinate System .Force Reference Frame Global Coordinate System Instantaneous Center of Rotation Instantaneous Point of Reaction Intervertebral Disc . Musculoskeletal Research Laboratory Motion Segment Unit Neutral Zone Posterior Longitudinal Ligament Pure Moment Range of Motion .Rotational Range of Motion Real-Time Load-Control .Standard Deviation Tracking Error . Trunk Weight University of Tennessee Health Science Center 


\section{CHAPTER 1. INTRODUCTION}

The centerpiece support mechanism of the human body is the spine, simultaneously providing protection for the spinal cord and structural support while enabling mobility for mechanical activity. ${ }^{1}$ The consequences of compromises in spinal health frequently include pain, some level of disability, imperative physical therapy, medication, and often surgery. ${ }^{2}$ As the population increases and ages, spine-related medical cases grow in frequency and the basis builds for mandated improvements in current remediating techniques. ${ }^{3}$ In order to solve the problem of compromised spinal health one must first understand the baseline of spinal health and optimal performance. In this case, a thorough knowledge of the kinematic behavior of the spine is required to understand the effects of specific conditions, injuries, and surgeries. That knowledge is also foundational for proper design of functional spinal implants made for injury prevention and tissue replacement. ${ }^{2,4}$

In vivo loads on the spine are complex and impossible to measure without compromising tissue with invasive measurement equipment. ${ }^{5}$ So instead of simulating in vivo loading scenarios, early biomechanists investigated fundamental structural properties. Promoting repeatable methodologies which could produce data for quantitative comparison between laboratories and testing platforms, the idea of "isolated" loading was applied across the field of 'bench-top' biomechanical testing. ${ }^{6}$ Conventional spinal biomechanics focused on applying singular bending moments on the spine while holding all other forces and moments to zero in what is called a 'Pure' Moment (PM) protocol. Variations of this protocol incorporate the coordinated application of compressive load ${ }^{7}$ to increase stability ${ }^{8,9}$ and to simulate muscle forces ${ }^{10,11}$ thus taking steps from what was initially a structural property study towards in vivo load recreation. One of the most popular variations is called the Follower Load (FL) protocol (Section 2.4). ${ }^{12}$ The PM and FL protocols are considered the gold standard testing protocols in spine biomechanics because data from these protocols are used in tissue resection studies investigating soft tissue component contributions, ${ }^{13}$ in surgical procedure and implant investigation studies, ${ }^{14,15}$ and in mathematical model development. ${ }^{16,17,18,19}$ These protocols offer repeatable methodologies with typical results, and there are many different mechanisms ${ }^{20}$ of application but there are also downsides.

The PM protocol has reduced the physiological loading environment to a uniplanar testing procedure for the sake of repeatability and comparability, however these compromises raise concern. Physiologic spinal motion occurs in multiple planes and involves many different component forces. ${ }^{1,2,4,21,22}$ Furthermore testing an implant to investigate its potential in vivo performance by applying a protocol that, rather than recreating in vivo conditions, simplifies and isolates loads, would likely reveal data that are inconclusive with regards to in vivo performance. ${ }^{14}$ Also, although the FL is a step toward closing the gap, further limitations exist at the platform level where application mechanisms limit certain motions and the controllability of applied forces, ${ }^{6,9}$ not to mention the lack of a concrete empirical benchmark for in vitro recreation of in vivo loads. While many current approaches are well-established, well-documented, and well- 
understood, the arena of robotics may offer substantial improvements to biomechanical testing in repeatability, controllability, and protocol innovation.

The use of robotics in biomechanical testing has been long established. ${ }^{23}$ Now many researchers employ robotic testing platforms, some of which are custom built ${ }^{10,18}$ while some are purchased and then modified for biomechanical testing. ${ }^{6,24,25}$ A frequently imitated platform characteristic in the robotic biomechanical testing community is realtime load-control (RTLC). This mechanism for protocol application involves the use of force and moment data as feedback and will be defined more precisely in later Section 2.4.2. However to the author's knowledge, there has been limited published application of load-controlled robotic platforms outside the simulation of gold standard biomechanical protocols.

The next step in robotic biomechanical testing is to develop a platform validated in gold standard protocols with the capability of enabling development of innovative protocols while overcoming conventional robotic testing platform limitations. A more general purpose for this development is to close the gap between the in vivo and in vitro environments.

The first objective of this thesis was to introduce a methodology for applying a dynamic force vector utilizing a novel robotic biomechanical testing apparatus. The chosen application of this method was the robotic simulation of an ideal FL (the standard non-pure moment testing protocol for which a force of constant magnitude but dynamic direction is requisite). The second objective of this thesis was to quantitatively compare two gold-standard protocols, PM and ideal FL and one novel protocol involving the intentional application of shear in combination with compression. The spinal kinematic characterizing metrics used to compare the protocols were flexion/extension rotational range of motion (RROM) and anatomical point translation (APT), a novel quantity used for comparison of a single L4-L5 spinal motion segment. 


\section{CHAPTER 2. BACKGROUND}

Chapter 2 is comprised of four sections. Section one addresses the anatomy of the spine with specific attention to the lumbar region. Section two discusses the basic mechanics of the spine. Section three generally describes the kinematics of the lumbar spine and section four introduces biomechanical testing and its relevant application to the study of the lumbar spine.

\subsection{Anatomy of the Spine}

The human spine, running from the base skull to the coccyx is comprised of 25 separate vertebral bodies arranged in a columnar fashion and divided into four sections denoted by four different curvatures: the cervical lordosis, the thoracic kyphosis, the lumbar lordosis, and the sacral kyphosis. ${ }^{2}$ Figure 2.1 identifies these regions and displays the number of vertebrae in each section, seven cervical bodies, twelve thoracic bodies, five lumbar bodies, and eight to nine sacrococcygeal bodies which are displayed as a single body because they are typically fused together. ${ }^{22,26}$ The spine can be broken down into functional units which are composed of two adjacent vertebral bodies, the conjoining intervertebral disc (IVD), and the surrounding ligaments and muscles, hereafter referred to as a motion segment unit (MSU). ${ }^{27}$

\subsubsection{The Vertebra}

The vertebrae, of which an example may be found in Figure 2.2, are the bony structures of the spine to which ligaments and muscles via tendons attach. Biologically these structures are responsible for the protection of the spinal cord and are partially responsible for the nutrition of the IVDs which are primarily avascular (Section 2.1.2). Mechanically, vertebrae are responsible for transferring load while providing stability and guiding motion. The form of the anterior portion (body) primarily addresses load displacement while the structures of the posterior portion mainly deal with motion guiding. The body is contacted superiorly and inferiorly by the cartilaginous endplates of IVDs through which the vasculature of the cancellous bone of the body is able to deliver nutrients to the disc. Lumbar vertebrae have relatively large bodies when compared to cervical and thoracic vertebrae, a characteristic mainly attributed to the greater loads borne by these bodies. ${ }^{2,4,27}$

The posterior structures of a vertebra include the pedicle, lamina, transverse processes, spinous process, and the zygapophyseal joints (facets). The left and right pedicles connect the lamina to the posterior portion of the vertebral body creating the vertebral foramen through which the spinal cord runs along the entire length of the spine. ${ }^{2}$ The transverse and spinous processes act as moment arms for the attachment of muscles and ligaments. ${ }^{4}$ 


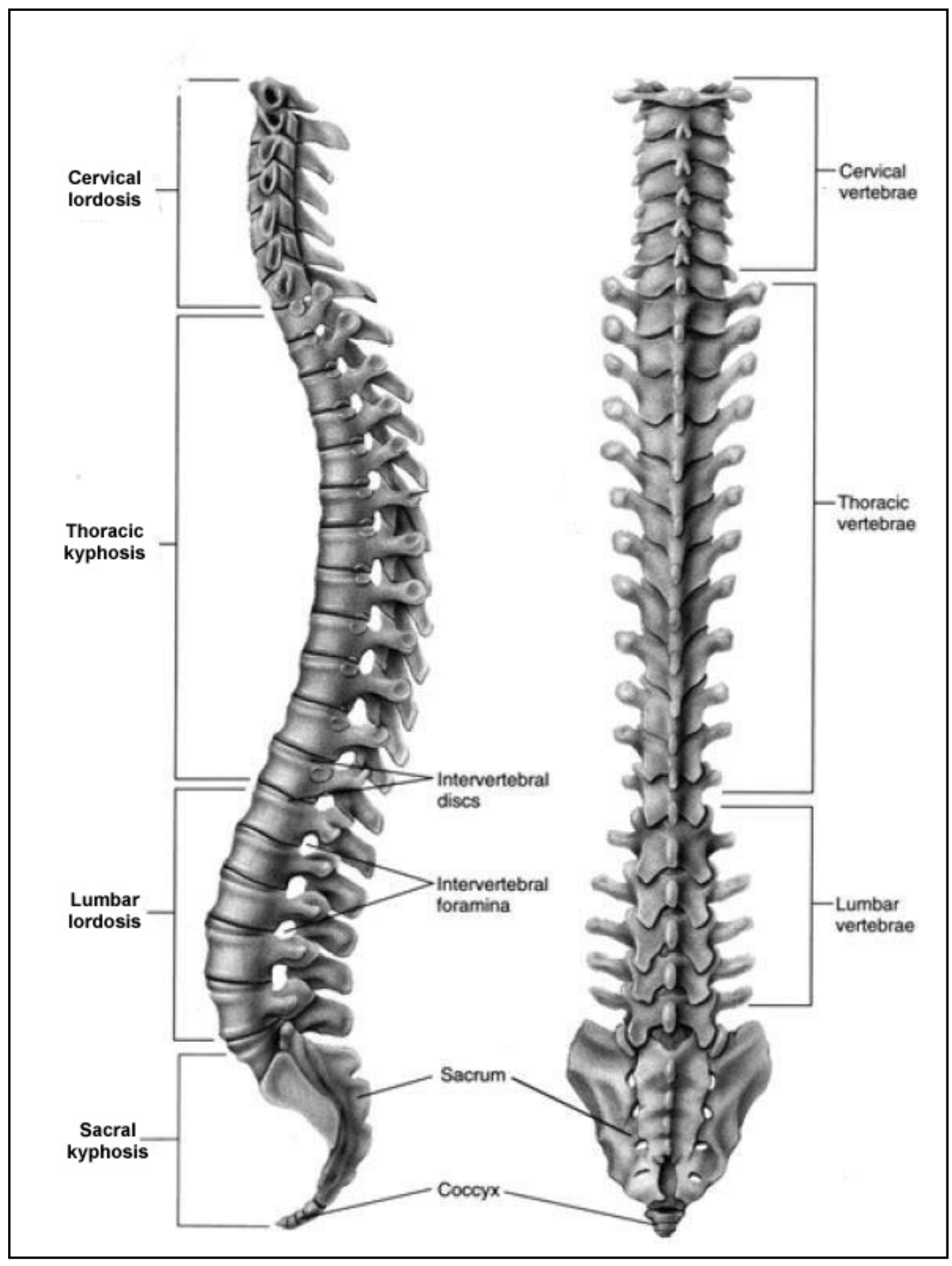

\section{Figure 2.1 The human spine}

The lateral (left) view displays the four curves of the spine that act as regional definitions which are otherwise more difficult to discern in the posterior (right) view. Adapted with permission from J. Butler, J. W. Hole, R. Lewis and D. Shier, 2002. Hole's human anatomy \& physiology, 9th ed. McGraw-Hill, Boston, p $156 .{ }^{26}$ 


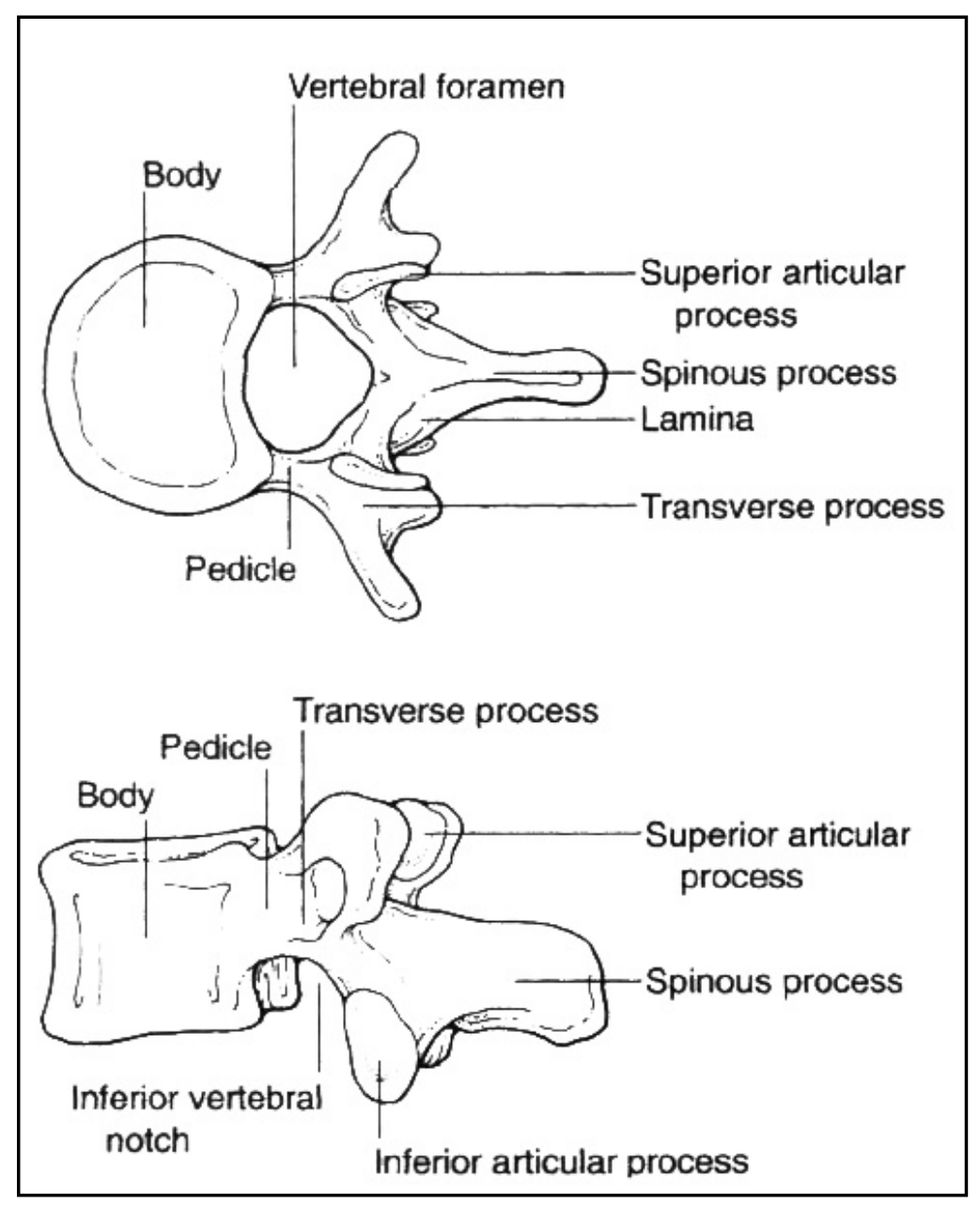

\section{Figure 2.2 A vertebral body}

Superior (top) view adequately shows the delineation between the vertebral body and the posterior structures. Lateral (bottom) view shows the alignment of the superior and inferior articular processes. Reprinted with permission from E. C. Benzel, 2005. Spine surgery : Techniques, complication avoidance, and management, 2nd ed. Churchill Livingstone, Philadelphia, Pa., p 78. ${ }^{28}$ 
The facet joint between adjacent vertebrae is comprised of the superior vertebra's inferior articulating surface and the inferior vertebra's superior articulating surface. This synovial joint glides on articular cartilage guided by meniscus-like fat pads, within a highly innervated capsule rendering this joint a functional proprioceptor as well as a source of pain. The joint mechanically provides stability by limiting motion in extension, lateral bending, axial rotation, and vertebral body translation with respect to adjacent bodies (Section 2.2.5), but allowing coupled motion between lateral bending and axial rotation. ${ }^{2}$

\subsubsection{The Intervertebral Disc}

The IVD is situated between the bodies of two adjacent vertebral bodies and functions by absorbing and dispersing compressive and shear loads between vertebrae (Section 2.2.1). It is divided into three sections, the nucleus pulposus, annulus fibrosis, and the cartilaginous endplate ${ }^{4}$ as seen in Figure 2.3. The fibers that make up these three sections, however, are not distinctly demarked and are continuous across boundaries. ${ }^{29}$ The disc is anatomically the same at every level but varies in shape and size, conforming to neighboring vertebral bodies which are subject to different loading scenarios. ${ }^{2}$

The nucleus pulposus is a hydro-gelatinous matrix of proteoglycans, loose type II collagen fibrils, water, ions, and trace cellular particulates. The proteoglycans utilize characteristic negative charges to attract ions creating an osmotic gradient and subsequently drawing in water through osmosis. ${ }^{30}$ This influx of water and the hydrophilic nature of the proteoglycans pressurize the disc. This capsule-like design of the disc allows it to convert axial force to radial force functioning as a shock absorber. Disc pressure is directly related to applied forces and nuclear water content, the latter of which depends on factors like physical activity, injury, gender and age. A decrease in disc pressure does not fully hinder load distribution capabilities but does significantly affect motion and proper function.

The annulus fibrosis is made up of concentric sheets of type I and type II collagen fibers aligned obliquely to the longitudinal axis of the spine in a nearly orthogonal, alternating fashion. Annulus fibers attach to the cartilaginous endplates superiorly and inferiorly with the exception of the outermost Sharpey Fibers, which attach directly to the calcified ring apophysis of the adjacent vertebral bodies. The fibers encapsulate the nucleus material facilitating pressurization and absorbing the radially converted force by resisting in tension (Section 2.2.1).

The cartilaginous endplates are remnants of fibrocartilaginous growth plates from childhood. They are porous in structure, facilitating nutrient transport. And they cap the annular capsule superiorly and inferiorly, connecting annular fibers to the vertebral bodies. $^{2}$ 


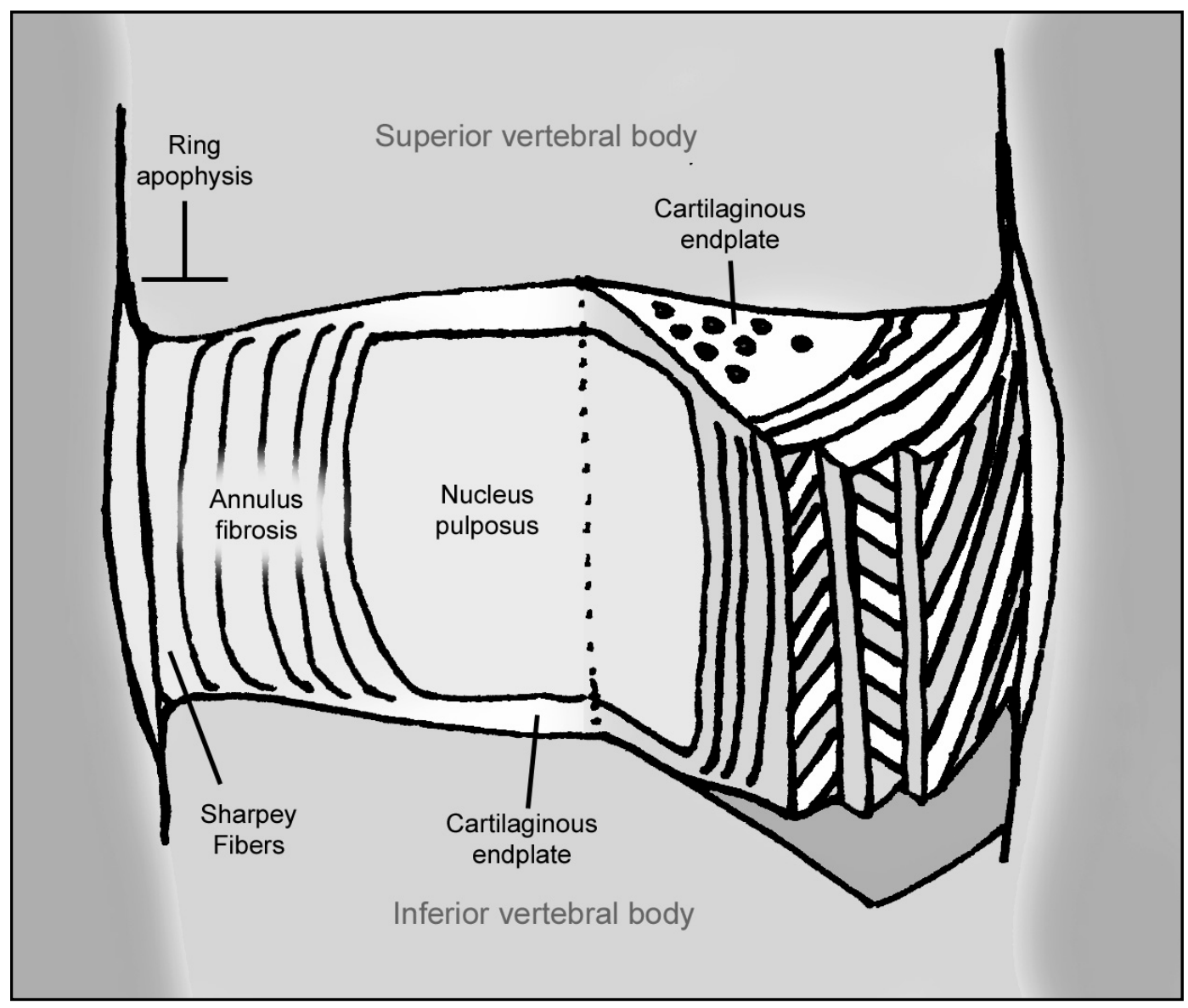

Figure 2.3 The intervertebral disc

A non-scaled example of an IVD. 


\subsubsection{Ligament}

A ligament is a fibrous connective tissue that serves to connect bone to bone and function by limiting or guiding motion. Ligaments can only withstand tensile forces and are made of collagen fibers, which resist tensile forces greatly, and elastin fibers, which elongate slowly affording mobility. Ligaments are generally unidirectional and will buckle under compressive loads. The ligaments involved in the lumbar spine are represented in Figure 2.4.

Two primary ligaments run the full length of the spine, the anterior longitudinal ligament (ALL) and the posterior longitudinal ligament (PLL). Both ligaments are broad ligaments loosely attaching to the periosteum of vertebral bodies and strongly attaching to the annular tissue. The ALL runs along the anterior of the column of vertebral bodies while the PLL runs along the anterior surface of the vertebral foramen on the posterior surface of the column of vertebral bodies.

Other spinal ligaments cross single MSUs: facet capsular ligaments, ligamentum flavum, the intertransverse ligaments, and the interspinous and supraspinous ligaments. Facet capsular ligaments connect the articulating processes across the joint, defining the formation of the synovial capsule. The ligamentum flavum runs along the posterior of the vertebral foramen and is commonly referred to as the most flexible ligament in the body. ${ }^{2}$ The intertransverse ligaments connect right and left transverse processes superiorly and inferiorly to the corresponding processes on adjacent bodies. The interspinous and supraspinous ligaments connect spinous processes of adjacent bodies.

\subsection{Mechanics of the Spine}

\subsubsection{Mechanics of an IVD}

As mentioned before, the IVD acts as a shock absorber as seen in Figure 2.5B. The hydrostatic pressure generated by the nucleus and contained within the annulus converts axial loads normal to the endplate into radial loads, distributing them about the annular fibers which then experience tension. This tension allows fibers that would otherwise buckle under compression to limit mobility of the disc in a fashion that stabilizes the spine under commonplace loading scenarios such as supporting body weight. ${ }^{4}$

The IVD also acts as a stabilizer under lateral and sagittal bending. The anatomy of the disc facilitates stability during compression via redirecting forces to be borne via tension, but the fibers of the disc also function to withstand tension on the disc. This is apparent in the application of bend as seen in Figure 2.5B, where one side of the disc experiences compression while the other experiences tension. This allows the disc to not

only act as a compressive shock absorber but as a rotational stabilizer. ${ }^{2}$ Various ligaments and the facet joints also act to limit and guide motion. ${ }^{4,22}$ 


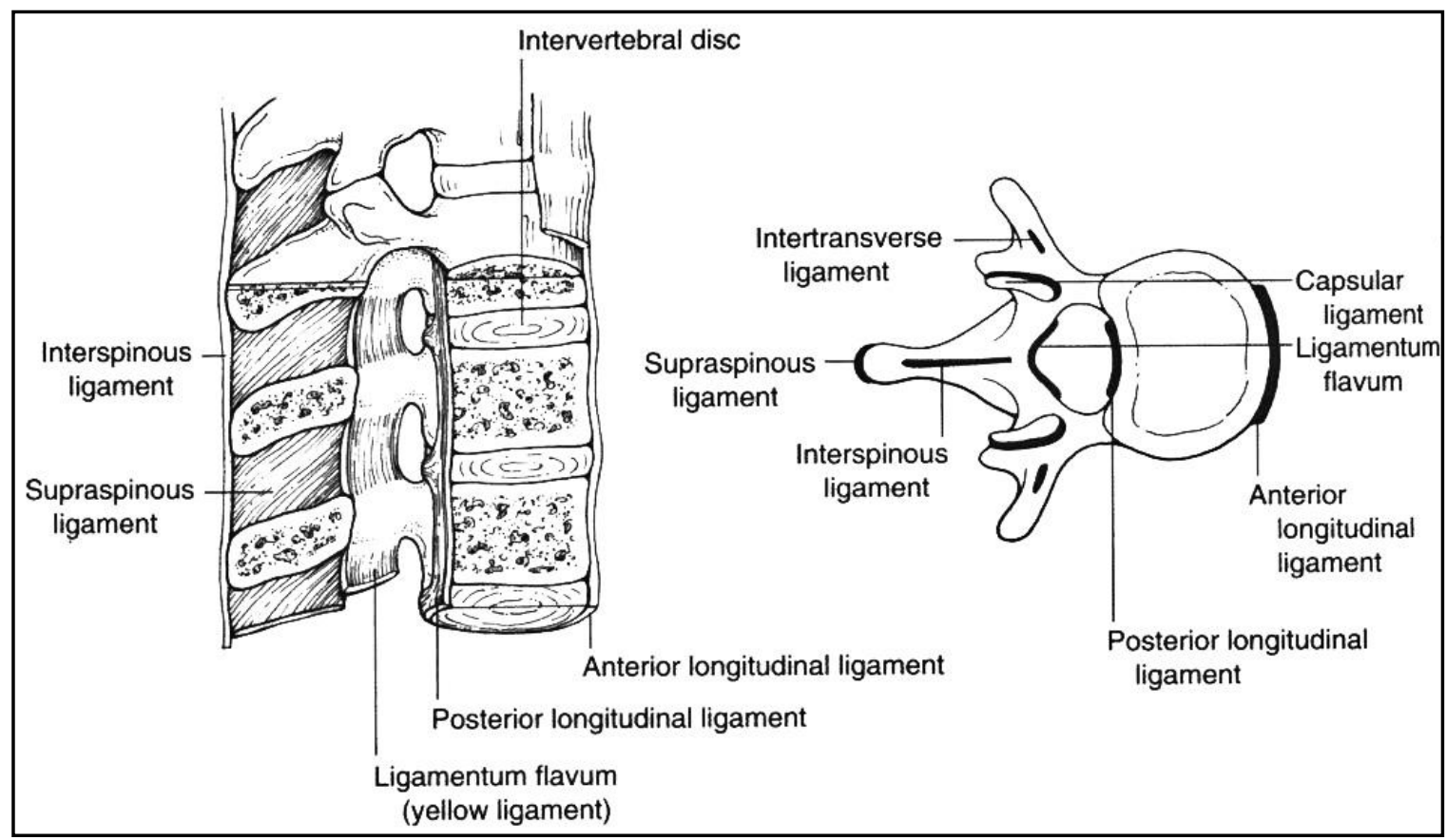

Figure 2.4 Various ligaments of the spine

Reprinted with permission from E. C. Benzel, 2005. Spine surgery : Techniques, complication avoidance, and management, 2nd ed. Churchill Livingstone, Philadelphia, Pa., p 78. ${ }^{28}$ 


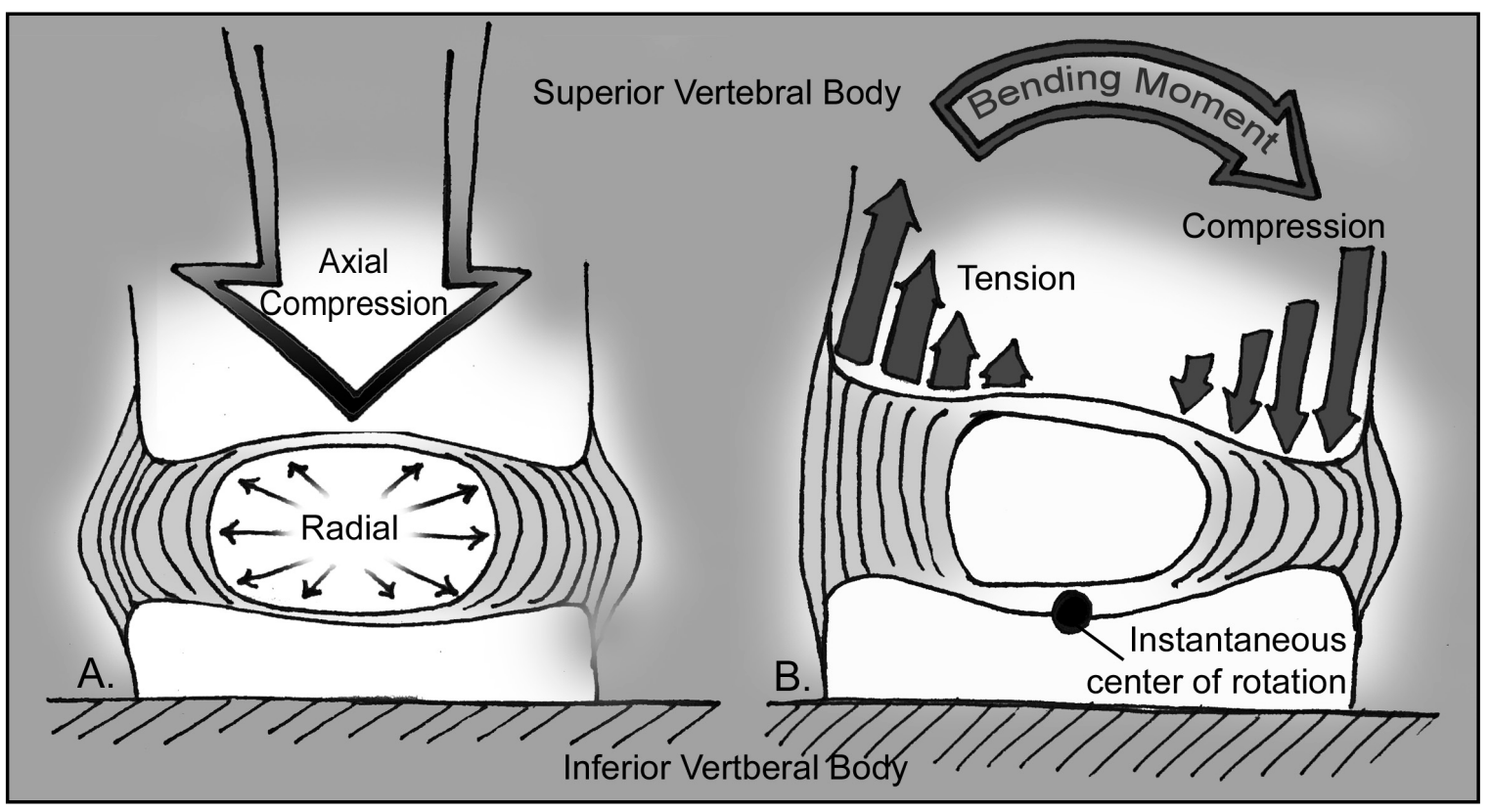

Figure 2.5 Basic IVD mechanical response to loads

In response to compressive loads (A) the nucleus pulposus contained within the annulus fibrosis redirects the compressive load radially, placing the annular fibers in tension, whereas in response to bending moments (B) the nucleus and annulus work together to resist the load, with one side of the annulus in tension from the vertebral bodies and the other side in tension from the nucleus.

Source: A. A. White and M. M. Panjabi, 1990. Clinical biomechanics of the spine, 2nd ed. Lippincott, Philadelphia. 


\subsubsection{Mechanics of the Lumbar Spine}

The in vivo loading conditions of the lumbar spine are complex but commonly simplified. The main consideration is the mass of the trunk weight supported by the spine. In most instances the trunk's center of mass (COM) is anterior and superior to the lumbar spine but, depending on body type and weight, it can be located elsewhere. This mass is acted upon by gravity and is represented by the force $\left(F_{B W}\right)$ displayed in Figure 2.6A. The perpendicular distance between $F_{B W}$ and the instantaneous point of reaction (IPR) within the joint dictates the moment. The postural muscles attached to the posterior structures of the vertebrae play a part in counteracting this moment along with the IVD. The curvature of the spine causes each MSU to see a different moment value. ${ }^{2,4,31}$

Not only does this body mass force apply a moment to the lumbar MSUs but forces as well. Within the scope of a single level, as in Figure 2.6B, the body mass force has an axial component directed normally through the mid-plane of the disc $\left(F_{N}\right)$ and a shear component parallel to the mid-plane of the disc $\left(F_{S}\right)$. The magnitudes of these components are affected by the mass of the trunk and the angle of the mid-plane of the disc with respect to the global horizontal plane $(\theta)$, which determines the direction of gravity. These component forces can by calculated by using Equations 2.1 and 2.2:

$$
\begin{aligned}
& \mathrm{F}_{\mathrm{N}}=\mathrm{F}_{\mathrm{BW}} \cos \theta \\
& \mathrm{F}_{\mathrm{S}}=\mathrm{F}_{\mathrm{BW}} \sin \theta
\end{aligned}
$$

When considering the sagittal plane, for instance, theta may change by flexion or extension, affecting the magnitude of the shear component as well as the direction of that shear in the global perspective. When considering the local, disc-oriented perspective, the shear component is always directed along the mid-plane of the disc and therefore never changes direction, only magnitude. It is important to note however that under bending, the magnitude of the shear force component changes, suggesting a possible change in response to load. ${ }^{4}$

The shear force causes the translation of a superior vertebral body with respect to the inferior one, biomechanically referred to as listhesis. ${ }^{2}$ This quantitative metric for measuring spine kinematics is notable for its absence in comparative literature concerning spine biomechanics. Drastic listhesis is a behavior that is related to spinal

instability ${ }^{2}$ suggesting the reason that there is limited published data on specific quantities in healthy MSUs.

\subsection{Kinematics of the Lumbar Spine}

Kinematics is the area of mechanics involved the study of motion of rigid bodies without specific concern for the causal loads. In spinal biomechanics there is debate between the perspectives used to address those loads but it is nevertheless important to note that motion is caused by load. Spine kinematics will henceforth be discussed 


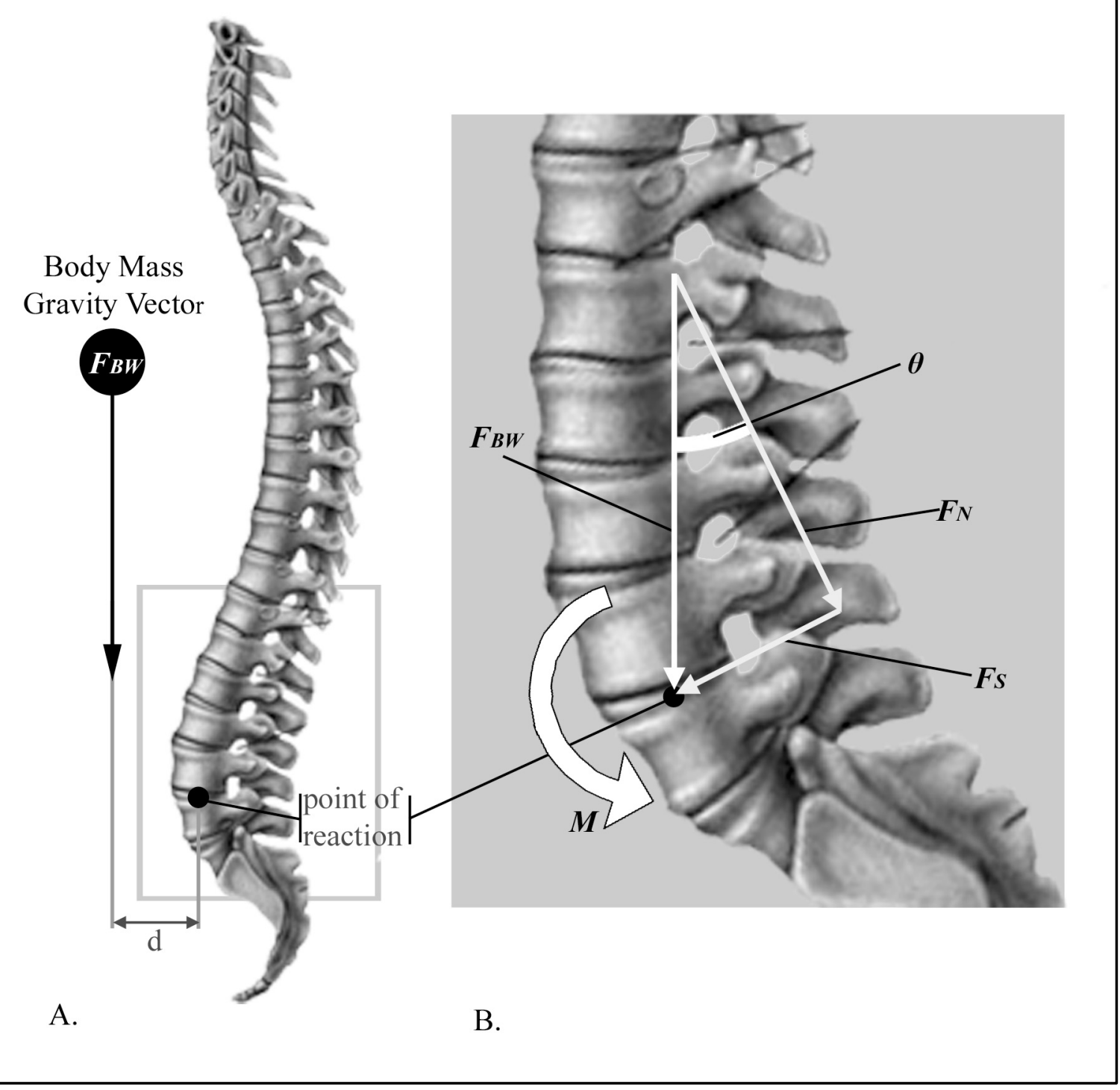

\section{Figure 2.6 Simplified mechanics of the spine}

The gravitational force $\left(F_{B W}\right)$ applied to the COM $(\mathrm{A})$ is directed vertically in the global force reference frame. It induces a rotational moment $\left(M=F_{B W} * d\right)$ at each MSU level (B). The vertical force has components normal $\left(F_{N}\right)$ and parallel $\left(F_{S}\right)$ to the disc at a specific level whose magnitudes are functions of body weight and disc angle $(\theta)$. The rotational moment and component forces are different at each level because of variations in moment arm $(d)$ and disc angle $(\theta)$. The spinal segment in B has been rotated in order to exaggerate the disc angle and increase the shear force component for functional visibility. The spine image was adapted with permission from J. Butler, J. W. Hole, R. Lewis and D. Shier, 2002. Hole's human anatomy \& physiology, 9th ed. McGraw-Hill, Boston, p $156 .^{26}$ 
regarding vertebral bodies as rigid bodies while motion is characterized by the position of the mobile superior (top) body with respect to the fixed inferior (lower) body. Spinal MSUs possess six degrees of freedom (DOF) of motion as seen in Figure 2.7. They are accounted for as three translational degrees along three orthogonal axes and three rotational degrees about those same three axes. Each degree of mobility has a specific characteristic response to load and some degrees have coupling effects due to vertebral geometry. The mechanisms governing response to load involve soft and hard tissues rendering the response dynamic and characteristically viscoelastic. These factors generate parameters in profiles of motion with respect to load like the neutral zone and effect the overall range of motion (ROM) in specific DOF. ${ }^{4}$

\subsubsection{Neutral Zone}

The neutral zone (NZ) in any degree of freedom is defined by Panjabi as the region of rotation between the neutral position and the point of spinal resistance to physiological motion first encountered during bending. The NZ is theoretically the region of motion that has low resistance, requiring small magnitudes of load to cause motion. This is a common metric for comparison of data in publications ${ }^{9,21}$ however the quantitative definition of such a parameter may not be appropriate. Although the NZ is considered in cases of degenerative disc disease, trauma, and surgery it was originally defined in a manner that leaves room for significant error due to the large difference between the in vitro loading scenarios used to define this parameter and the in vivo loading environment. ${ }^{32}$ Due to the limitations of conventional apparatuses used in biomechanical testing (Section 2.4.1) the current definition of this metric possesses an aspect of subjectivity. ${ }^{9}$

There is also suggestive evidence that the theoretical NZ of a relatively healthy, ligamentous spinal MSU is a much smaller region and approaches non-existence under simulated in vivo loading scenarios. Panjabi does go on to suggest a difference between passive and active NZs, the latter involving resting muscles, the former being exhibited by cadaveric tissue. He also comments on their differences that active NZs would likely be smaller than passive NZs measured in vitro. ${ }^{4}$ However this does not diminish the opportunity for a more objectively quantitative definition for NZ.

\subsubsection{ROM}

In response to loads ROM does not change the same way NZ changes but the two are not unrelated. ROM is easily defined as the distance between the two most extreme points of displacement in either translation or rotation. It is a metric used in medical diagnoses and is the most commonly used metric of comparison in spinal biomechanics. A common practice is to compare pre- and post-surgical procedure ROM values of a cadaveric specimen. $2,4,6,9,14,16,21,22,33,34,35,36,37,38,39,40$ 


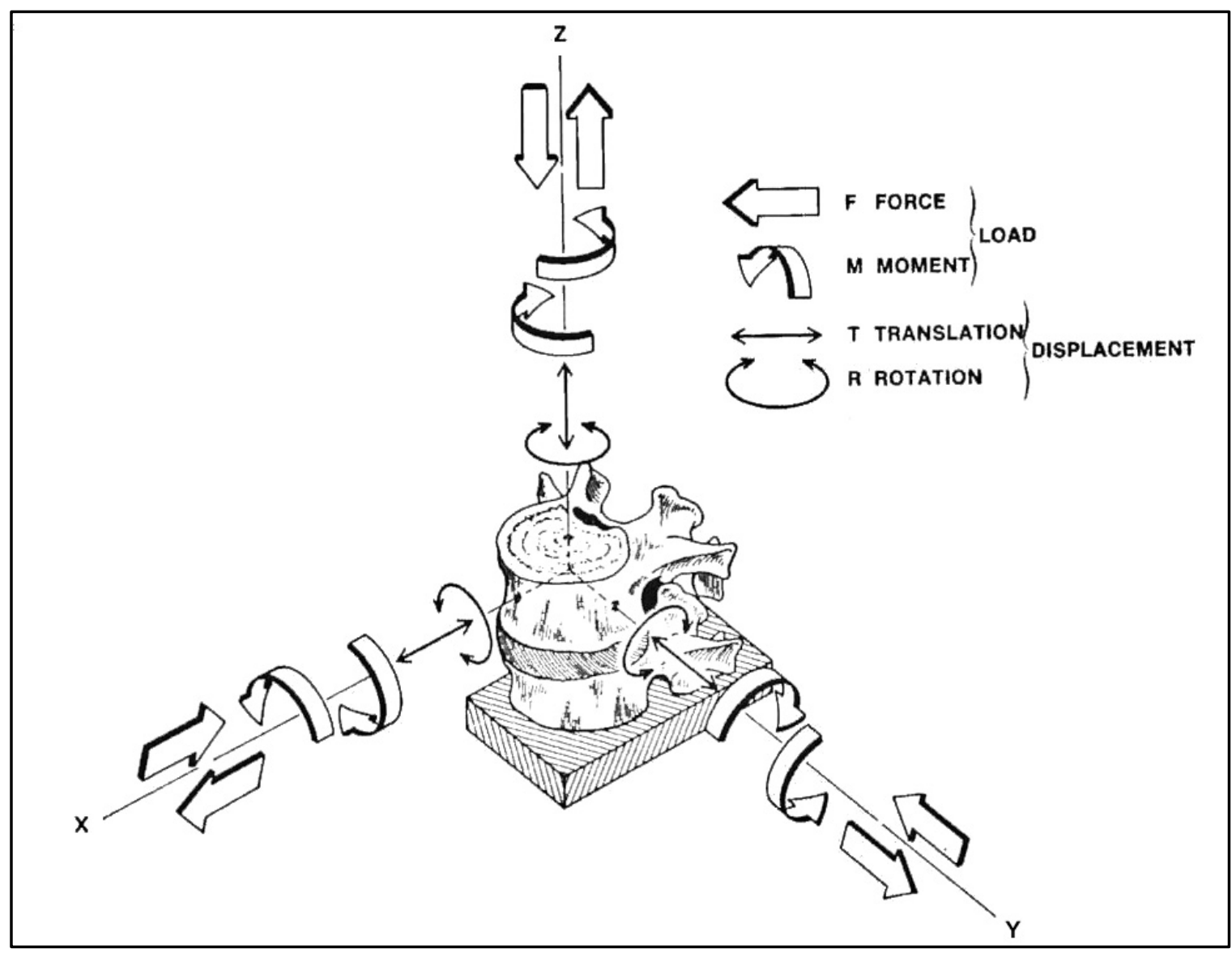

Figure 2.7 Spinal MSU degrees of freedom

Adapted with permission from A. A. White and M. M. Panjabi, 1990. Clinical biomechanics of the spine, 2nd ed. Lippincott, Philadelphia, p $25 .{ }^{4}$ 


\subsection{Biomechanical Testing}

Biomechanical testing of the spine is conducted in many laboratories across the world utilizing various mechanisms for investigations. Main goals of spinal biomechanics include obtaining better understanding of the in vivo mechanical properties and kinematic characteristics of the spine and determining the direct and indirect as well as immediate and prolonged effects of certain pathologies and surgical procedures on those properties and characteristics. ${ }^{7,9,21,24,25,41,42,43}$ Unfortunately, directly observed in vivo data are remarkably sparse, commending in vitro biomechanical testing. ${ }^{7,14}$

\subsubsection{Flexibility versus Stiffness Testing}

In 1988 Panjabi et al. developed a demarcation within biomechanical testing. Testing is separated by the primary independent variable, but not necessarily by the means of application. Protocols applying load and measuring displacement (translational, rotational, or both) would be flexibility tests while protocols actuating displacement and measuring reactive loads would be stiffness tests. There is still debate among researchers over which methodology is more appropriate. ${ }^{6}$ One advantage of flexibility testing is that it possesses greater control over the complex loading environment of the spine, enabling testing platforms to produce typical data which are comparable across platforms, between laboratories, and between multi- and single-segment studies.

However this greater control has been applied in a limited fashion, mainly in the isolation of single loads, as in pure moment (PM) testing, where all forces and moments are targeted to $0 \mathrm{~N}$ and $0 \mathrm{Nm}$, respectively, with the exception of one planar moment. ${ }^{24,25,44}$ Also, flexibility protocol expansion via the introduction of combined loads still leaves supporters of stiffness testing resolute in their claim that controlling displacement yields better simulation of in vivo scenarios. Stiffness supporters argue that flexibility tests do not simulate the in vivo environment, and therefore cannot provide clinically relevant data on the behavior of the spine. ${ }^{4,31}$ The novel application of the not so novel field of robotics in the arena of biomechanical testing has the potential to offer researchers the control of flexibility testing and the realistic aspect of stiffness testing closing the gap between in vitro testing and in vivo conditions.

\subsubsection{Robotics in Biomechanical Testing}

Robotic testing platforms have been applied in the area of biomechanics nearly twenty years. ${ }^{10,24,25,37,38,44,45}$ Many of these platforms have attempted to perform flexibility testing by applying PMs to spine segments and MSUs. A primary tool developed for conducting these tests is load-control. Load-control in many concepts is simply varying load and measuring displacement.

For the rest of this thesis load-control will be defined as such: the use of force and moment measurements as commanded targets and as direct feedback for actuating 
mechanisms in real-time, meaning there is no discernible iterative step. While some laboratories claim load-control similar to this definition in reality they utilize positional data as feedback applying complex plant stiffness algorithms used to calculate a subsequent iterative displacement to obtain a desired force profile. ${ }^{25,45,46}$ This mechanism applies stiffness testing principles and mechanisms to produce kinematic data that resembles data obtained from flexibility testing. Published data on true RTLC exist but it is sparse and limited in application, only being validated in PM. ${ }^{24,44}$

The Musculoskeletal Research Laboratory (MRL) at the University of Tennessee Health Science Center (UTHSC) houses a custom-built, robotic, Cartesian, biomechanical test platform operating with 6 RTLC DOF as seen in Figure 2.8. It has been validated in flexibility testing of lumbar spine segments. ${ }^{47}$ Its robotic performance competes with the best published robotic biomechanical testing platforms and produces data that are comparable to published lumbar spine data. ${ }^{47}$ This testing platform will henceforth be referred to as the Cartesian Robotic Biomechanical Tester (CRBT). 


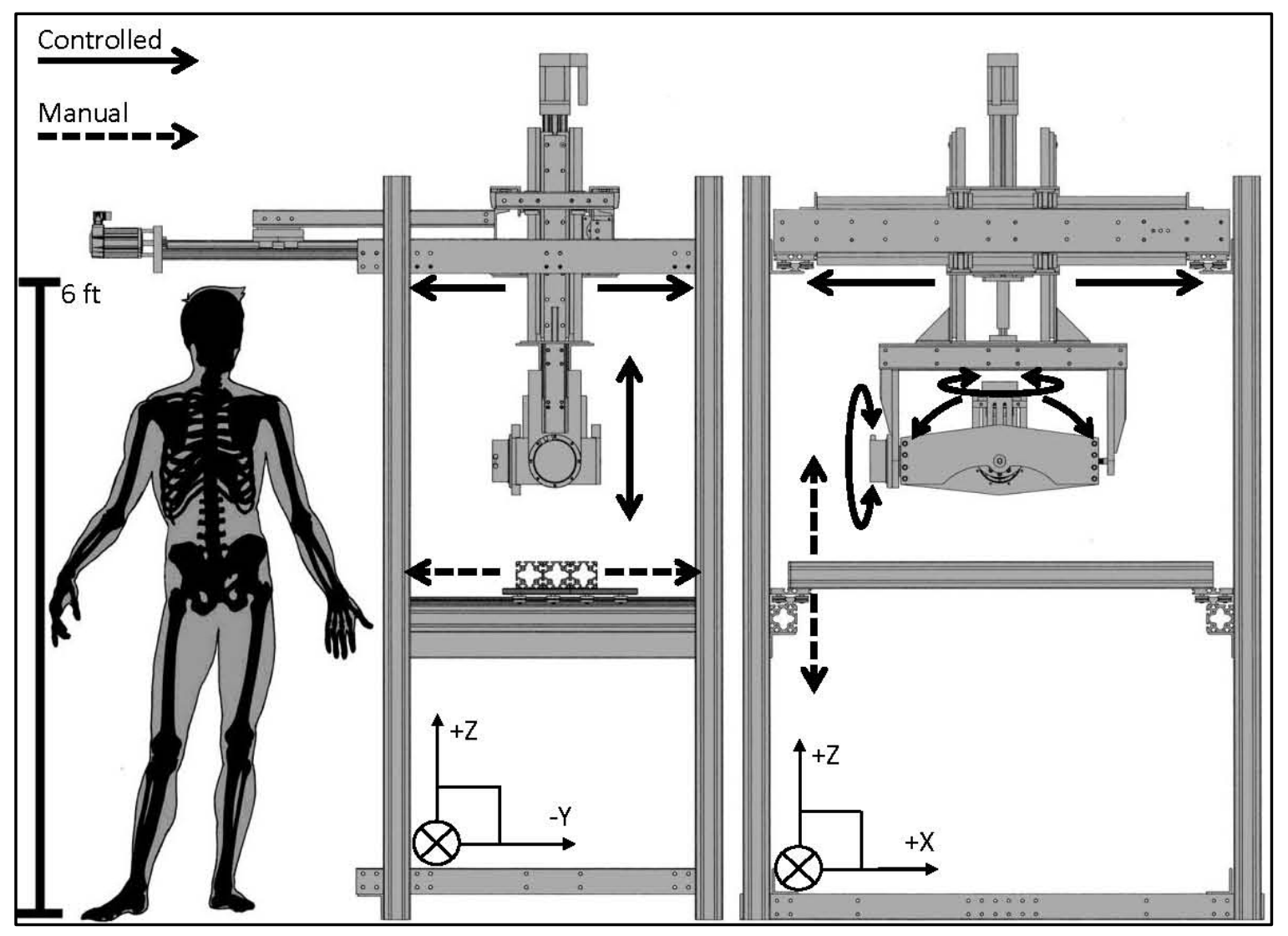

Figure 2.8 Custom built robotic biomechanical testing platform

The testing platform has six fully controlled DOF. The specimen is mounted on the manually mobilized table, which is locked during testing. The mobile gimbal then attaches to the "free" end of the specimen. For platform hardware and specifications, see Appendix A. 


\section{CHAPTER 3. ROBOTIC APPLICATION OF A DYNAMIC RESULTANT FORCE VECTOR USING REAL-TIME LOAD-CONTROL: SIMULATION OF AN IDEAL FOLLOWER LOAD}

The following text will comprise a technical note on a new methodology for applying a dynamic load vector to a lumbar spine segment and will validate the universal ability to apply a dynamic load vector during biomechanical tests with the CRBT.

\subsection{Introduction}

In vivo spinal loading conditions are comprised of dynamic force vectors. ${ }^{4}$ And although biomechanics has sought to isolate loading conditions for repeatable methodologies ${ }^{6}$ there still exists a need to close the gap between in vitro tests and in vivo circumstances where possible to better understand spine kinematics. In order to accurately test cadaveric tissue and any analogues, it would be advantageous to utilize a system capable of applying dynamic force vectors. Several currently used systems solve this problem with various cable-deadweight platforms. ${ }^{6,9}$ While current applications succeed at applying forces, some being indeed dynamic in nature, the area of robotics can provide some improvements in controllability and precision in applying those forces.

In order to mimic the in vivo condition of non-segmented motion, a form of loadcontrol is best suited for providing contiguous and dynamic load. Previous load-control attempts have applied incremental displacement in position-control to achieve specified load targets requiring complex matrices of plant stiffness and intensive calculation. ${ }^{25}$ Other attempts invoke genuine load-control, driving actuators directly with force error determined by commanded force targets. ${ }^{24,44,45}$ However these few attempts at robotic load-control have been limited to maintaining zero-load conditions, and even then some have large tracking error (TE) values. ${ }^{24,45}$

A robotic system comprised of independent axes operating in force or positioncontrol in coordination with the ability to apply a dynamic force vector is not unheard of, ${ }^{23}$ however the author is unaware of any such application to biomechanical testing.

The objective of this paper was to introduce a methodology for applying a dynamic force vector utilizing a novel robotic biomechanical testing apparatus. The chosen employment of this method was the robotic simulation of an ideal FL, the standard non-PM testing protocol for which a force of constant magnitude but dynamic direction is requisite. To the author's knowledge there has not been publication of a robotic, real-time load-control application of a non-zero load-vector during bending. This application not only sought to control a non-zero load vector during bending, but it also controlled a vector that was dynamic in its direction. 


\subsection{Materials and Methods}

Six fresh-frozen human cadaveric L4-L5 lumbar MSUs (male, mean age $44 \pm 8.9$ years standard deviation (SD)) were radiographically pre-screened for degenerative disc symptoms and osteophytes obstructive to normal anatomic motion, harvested and cleaned of excessive soft tissue. The segments were potted in bismuth alloy (Cerrobend ${ }^{\mathrm{TM}}$ ) in specimen-specific, natural lordotic alignment referenced to the assumed horizontal cranial endplate of L1, as seen in Figure 3.1, and then frozen until testing. The specimens were caudally fixed within the platform and cranially attached to the Cartesian manipulator.

The testing platform was comprised of a custom-built, Cartesian, RTLC Gantry system with six fully controlled DOF and a six-axis force-moment sensor (FMS) (Model 45E15A4-I63-AF JR3 Inc., Woodland, CA). Three harmonic drive motors comprising a roll-pitch-yaw (lateral bending, flexion-extension, axial rotation) gimbal assembly were suspended in series from three servo-actuated orthogonally oriented $\mathrm{x}$-(anteroposterior (AP)), y-(lateral), and z-(craniocaudal (CC)) translational axes which, collectively comprised a single Cartesian manipulator. ${ }^{10}$ The three translational axes comprised a stationary global coordinate system (GCS), to which all translational data were referenced. The FMS was rigidly mounted between the gimbal and cranial end of the specimen. The FMS also presented a local, mobile coordinate system (FMS-CS) in which all forces and moments were defined. Further use of these coordinate systems will be explained in the data processing segments.

All axes were controlled via an open-architecture, PID gain controller (UMAC Delta Tau Data Systems Inc., Chatsworth, CA) utilizing cascaded control loops and custom coded motion programs. A custom calibration algorithm was employed in realtime to correct for changes in FMS readings due to gravitational forces on attached pots and fixtures such that the platform directly controlled loads applied to the specimen. Complex equations for operation can be found in Appendix B. Each axis could operate utilizing position-control or load-control but regardless of that mechanism all movements were coordinated.

The ideal FL protocol is described in detail in Section 4.2 as a part of a full-length manuscript. This chapter was intended to be a short communication or technical note and as such the detailed explanation of the imitated protocol was cut in order to save words. For the sake of this section it is merely important to understand that when concerning a single MSU an ideal FL is applied normal to the disc, functionally minimizing any shear forces with respect to the disc.

The midline of the disc as defined by Figure 3.2 functions as the direction for ideal FL vector which is normal to the disc as shown in Figure 3.3. ${ }^{9}$ During sagittal bending the mid-plane of the disc rotates half as much as the cranial body therefore requiring the load vector to change direction while maintaining constant resultant magnitude. ${ }^{9,12}$ To input this dynamic load condition sagittal plane rotation was programmed to operate under constant velocity position-control while lateral and axial 


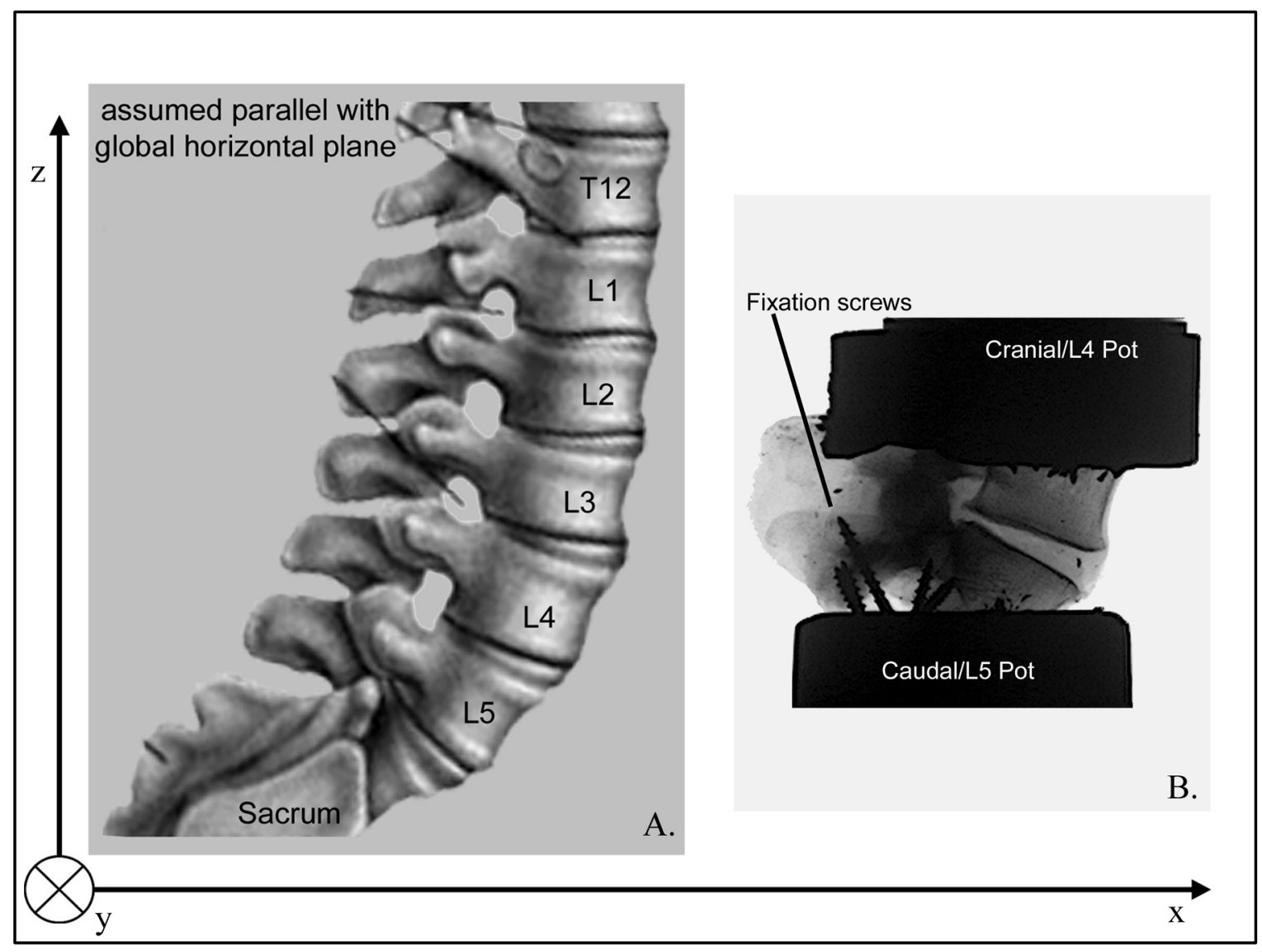

\section{Figure 3.1 Sagittal plane potting alignment}

The assumed horizontal cranial endplate (A) defines the angle of the disc with respect to the potting material (B). This angle is taken into account in the motion programs for controlling forces as well as any slight potting misalignments. See Appendix C for detailed motion programs. The spine image was adapted with permission from J. Butler, J. W. Hole, R. Lewis and D. Shier, 2002. Hole's human anatomy \& physiology, 9th ed. McGraw-Hill, Boston, p $156 .^{26}$ 


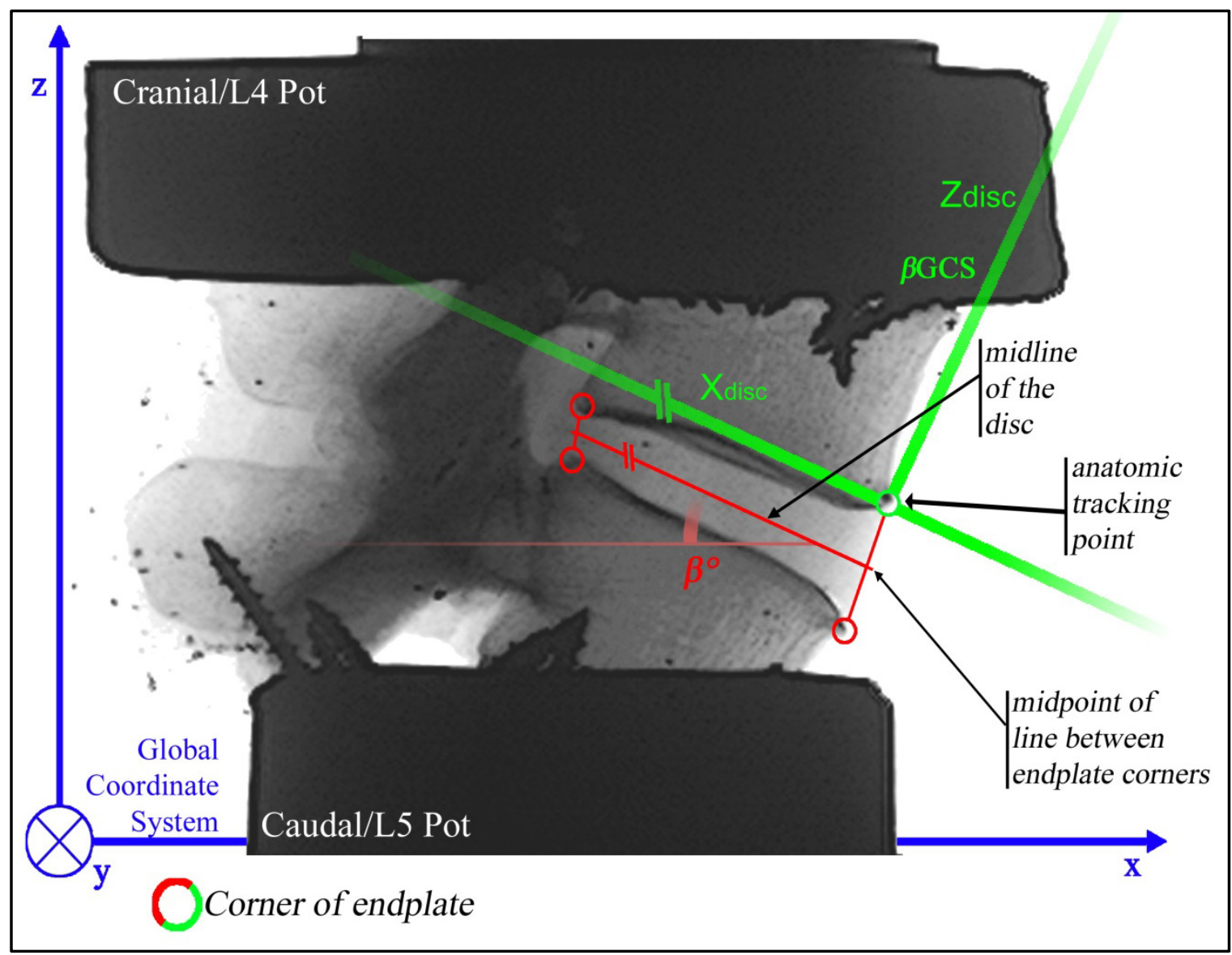

\section{Figure 3.2 Midline of the disc definition}

An example sagittal radiographic image of a potted specimen exhibits the location of the corners of the endplates adjacent to the L4-L5 IVD. Cross-disc lines (red) were drawn and marked to form the line indicating the midline (red) of the disc which had an angle $(\beta)$ with respect to the GCS (blue) horizontal while under no load. The $x_{d i s c^{-}}$and $z_{d i s c}$-axes are also denoted in green, forming the static $\beta \mathrm{GCS}$ (green), the coordinate system into which the APT data were transformed. The location of the anatomic tracking point when the specimen was under no load acted as the origin of the $\beta \mathrm{GCS}$. 


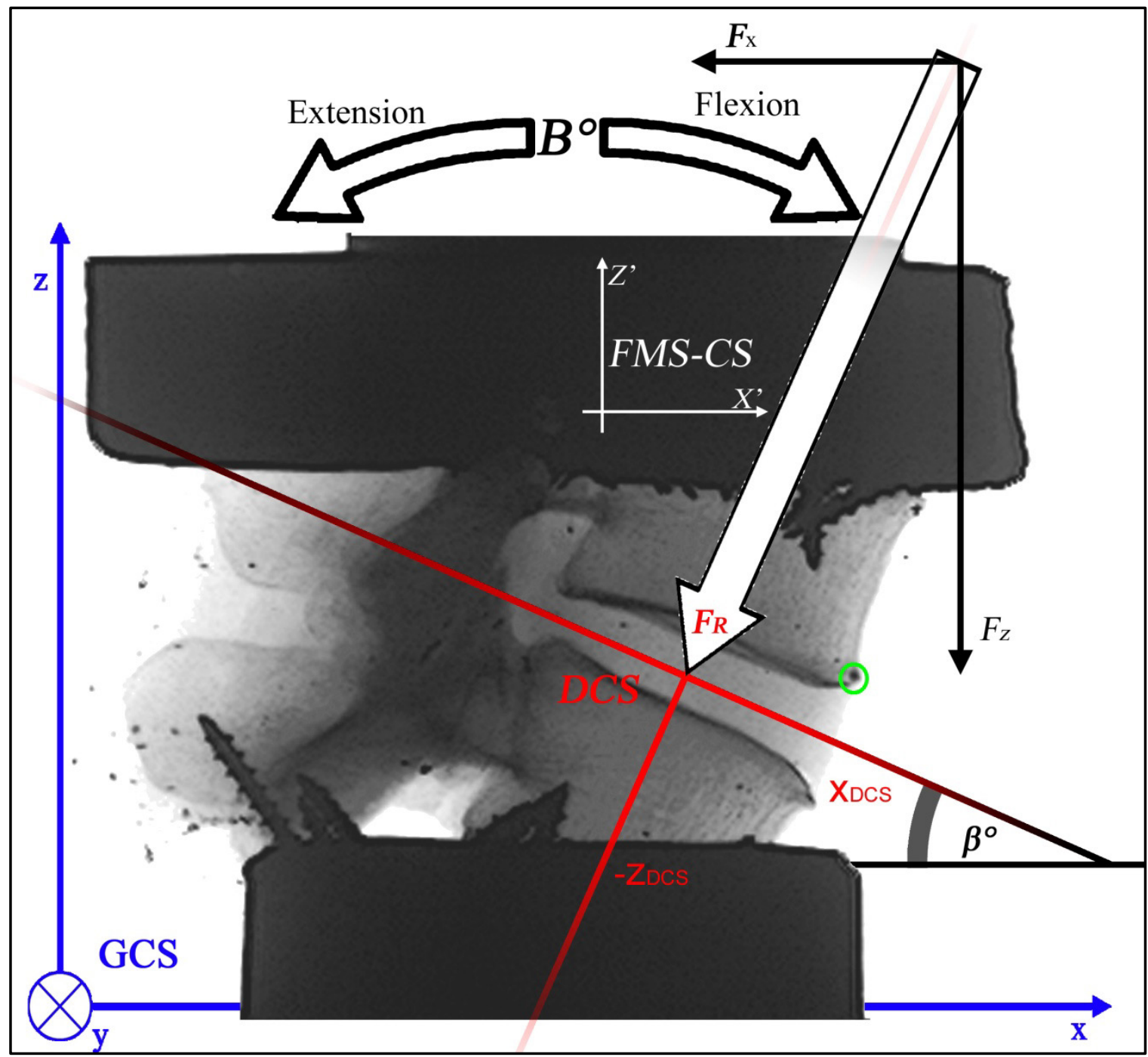

\section{Figure 3.3 Ideal FL application to single MSU}

The component forces $\left(\mathrm{F}_{\mathrm{x}}\right.$ and $\left.\mathrm{F}_{\mathrm{z}}\right)$ (within the FMS-CS, white) are dependent on the magnitude of the commanded resultant force $\left(\mathrm{F}_{\mathrm{R}}\right)$, the specimen specific sagittal potting angle $(\beta)$, and the mobile flexion/extension angle $(B)$. The resultant force remains normal to the also mobile mid-plane of the disc (defined by the DCS, red), which rotates at half the rate of the cranial pot because it is defined as the mid-plane between the mobile, cranial and stationary, caudal endplates of the L4-L5 disc. ${ }^{9}$ Also note the anatomic tracking point (green circle) used to define the displacement metric. The GCS is in blue. 
rotation axes operated in load-control to zero moment. All three linear axes operated under load-control with lateral forces controlled to a zero set point. A load magnitude of $400 \mathrm{~N}$ was chosen for simulation. ${ }^{48,49,50}$ At $0.2^{\circ}$ increment of sagittal rotation commanded FMS forces were thus programmed using Equations 3.1 and 3.2:

$$
\begin{gathered}
F_{x}=400 \sin (B / 2-\beta) \\
F_{z}=400 \cos (B / 2-\beta)
\end{gathered}
$$

where $B$ is the flexion/extension angle and $\beta$ is the angle of the mid-plane of the disc with respect to the top pot as seen in Figure 3.3. The resultant force was intended to have a constant magnitude when the force was referenced within the coordinate system defined by the midline of the disc (DCS). This coordinate system was a mobile coordinate system that was dependent on the sagittal rotation angle $(B)$. Based on the convention used to define the midline of the disc, the DCS rotated half as much at the FMS-CS in the sagittal plane during the test. Transforming the FMS-CS component forces to the DCS ideally would, in the case of the FL, make the $D C S_{X}$ force component non-existent and make the $D C S_{Z}$ component $400 \mathrm{~N}$.

To ensure applied forces did not build up initial moment artifacts a load vector was applied by sequentially applying $F_{z}$ and $F_{x}$ component forces while constraining flexion-extension rotation. Observed sagittal moment values in this state were due to the offset of net reactive specimen forces with respect to the FMS force reference frame (FRF). The FRF was transformed in the FMS-CS $S_{X}$ and $F M S-C S_{Z}$ directions to null these values and subsequent moment values were determined with respect to the newly located reference frame for all tests.

Specimens were mounted in the testing platform and manipulated to an unloaded state that delineated neutral angular orientation. The tissue was preconditioned by applying a flexion-extension PM protocol (all forces and non-sagittal moments commanded to $0 \mathrm{~N}$ and $0 \mathrm{Nm}$, respectively) to an $8 \mathrm{Nm}$ end-limit three times at a rate of $0.35 \%$ sec. The load vector was then applied to each specimen, which were subsequently extended and flexed (without pause) three times to an $8 \mathrm{Nm}$ end-limit at a rate of $0.35^{\circ} / \mathrm{sec}$ while the load vector was maintained in magnitude and direction with respect to the mobile mid-plane of the disc. Specimens were wrapped in moistened cloth to prevent tissue dehydration during testing.

Rotational, translational, force, and moment data were recorded at $10 \mathrm{~Hz}$ for analysis from the third bending cycle. Translational and rotational data along with fixturing and radiographic measurements were used to calculate the Cartesian translation data of the anterior-most point of the superior vertebral body's inferior endplate as noted in Figures 3.2 and 3.3. RROM and Cartesian displacement were analyzed under no load and neutral, flexed, and extended conditions with load. The observed AP and CC forces were combined to determine the resultant applied force for error calculation. 
The translational values were transformed from the GCS to a coordinate system $(\beta \mathrm{GCS})$ that was defined by the specimen specific sagittal disc angle when the specimen was under no load defined by the variable $(\beta)$. This coordinate system was a stationary coordinate system that acted a functional rotation of the GCS by the angle $\beta$. The rotated system was defined to emphasize the difference between shear (AP and $\Delta x_{\text {disc }}$ ) motion and compression ( $\mathrm{CC}$ and $\Delta z_{\text {disc }}$ ) motion. The $x_{d i s c}$-axis was parallel to the midline of the disc and the $z_{d i s c}$-axis was normal to the midline. The origin of the coordinate system was the location of the aforementioned anatomic tracking point when the specimen was under no load.

Using pixel counting software (Image-J National Institute of Health) a sagittal Xray image of the potted specimen was analyzed to determine the orientation of the midline of the disc. The anterior- and posterior-most points of the cranial and caudal endplates were located. Two cross-disc lines were drawn between the anterior points and posterior points. A single line was then drawn between the midpoints of the cross-disc lines. This line was designated to be the midline of the disc and is best displayed in Figure 3.2. The angle of this line was measured in relation to the assumed level bottom of the bottom pot to determine potted disc angle as seen in Figure 3.3. These measurements were conducted for every specimen with a 64 pixel per inch resolution.

\subsection{Results}

Force and moment values read by the FMS during a single test are exemplified in Figures 3.4 and 3.5. Average TEs across all specimens are displayed in Table 3.1. The average TE for the resultant force $\left(\mathrm{F}_{\mathrm{R}}\right)$ was $1.28 \mathrm{~N}( \pm 1.55 \mathrm{~N} \mathrm{SD})$ off the commanded force $(400 \mathrm{~N})$ across all specimens. Generally, the zero-commanded axes were held within a $\pm 0.1 \mathrm{Nm}$ range for moments and $\pm 1 \mathrm{~N}$ range for forces with maximum deviations not exceed $\pm 0.5 \mathrm{~N}$ and $\pm 5 \mathrm{Nm}$. Greater deviations from commanded values occurred when extension and flexion reversed direction however these deviations occurred outside the range of analysis.

The average RROM values for extension and flexion were $2.6^{\circ}\left( \pm 1.2^{\circ} \mathrm{SD}\right)$ and $5.0^{\circ}\left( \pm 1.7^{\circ} \mathrm{SD}\right)$, respectfully. The average APTs in the sagittal plane as result of initial loading, extension, and flexion were broken up into x-components and z-components in the $\beta \mathrm{GCS}$. The data can be found tabularized in Table 3.2 and graphically represented in Figure 3.6. The flexibility curve for a single specimen exhibits standard hysteresis behavior seen graphically represented in Figure 3.7. This data is often quantitatively evaluated in the literature, but due to the lack of standardized methods and frequent use of subjective methods, this data have been confined to evaluation in the qualitative arena. The platform was able to apply a load vector dynamic in direction and constant in magnitude. 


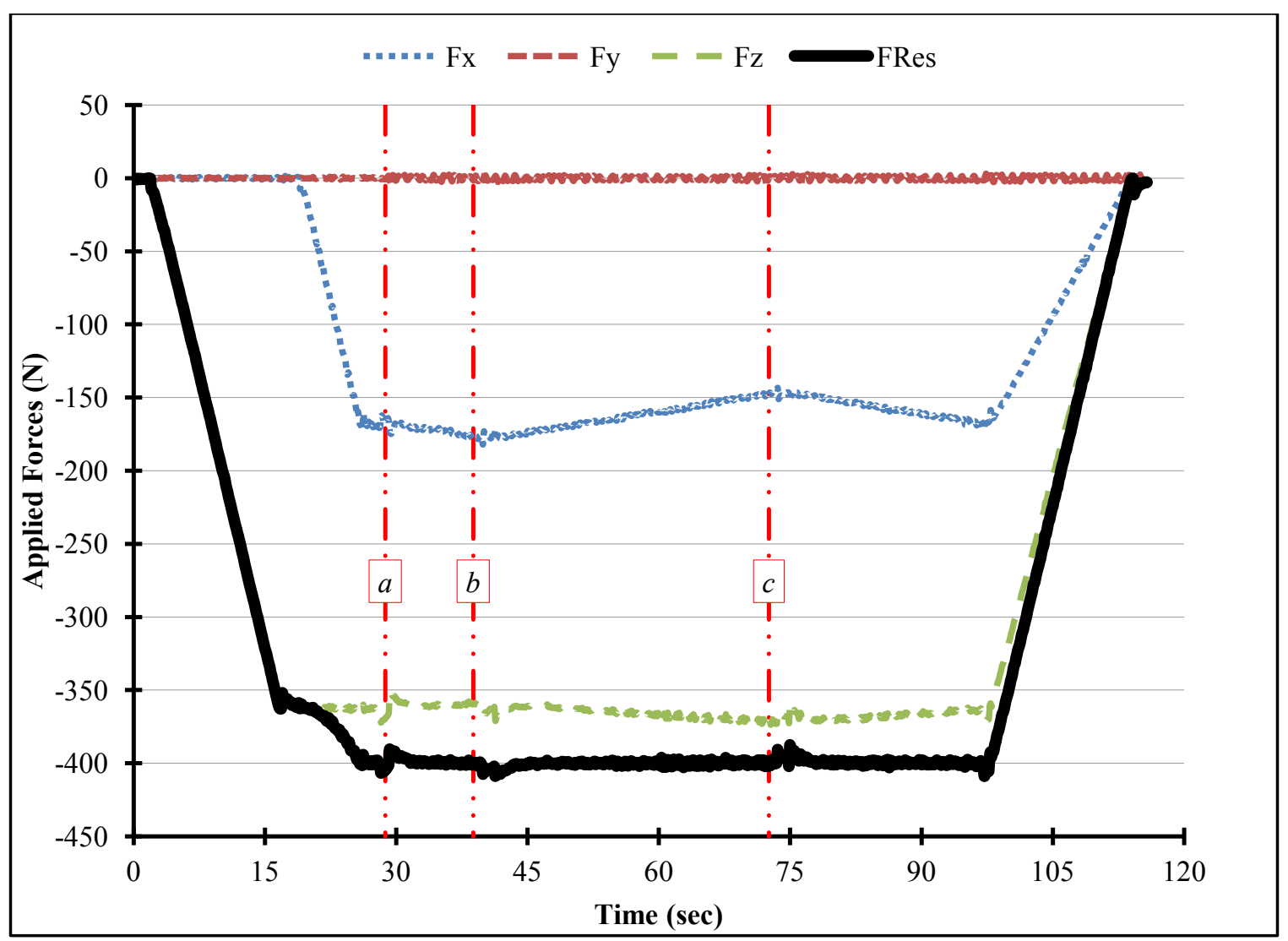

Figure 3.4 Graphical representation of applied forces

Lateral shear $\left(F_{y}\right)$ and resultant force $\left(F_{R}\right)$ were commanded to constant values of $0 \mathrm{~N}$ and $400 \mathrm{~N}$, respectively. It is dangerous, however, to define AP shear and CC compressive force as $\left(F_{x}\right.$ and $\left.F_{z}\right)$ because of the nature of their relationship involving the flexion/extension moment. Therefore, forces in the $\mathrm{x}$ - and $\mathrm{z}$-direction will be referred to as $F_{x}$ and $F_{z}$, respectively. RROM and displacement data were collected for analysis at instances FL load without bending or $0 \mathrm{Nm}(a), 8 \mathrm{Nm}$ extension $(b)$, and $8 \mathrm{Nm}$ flexion (c).The data were collected at a frequency of $10 \mathrm{~Hz}$. 


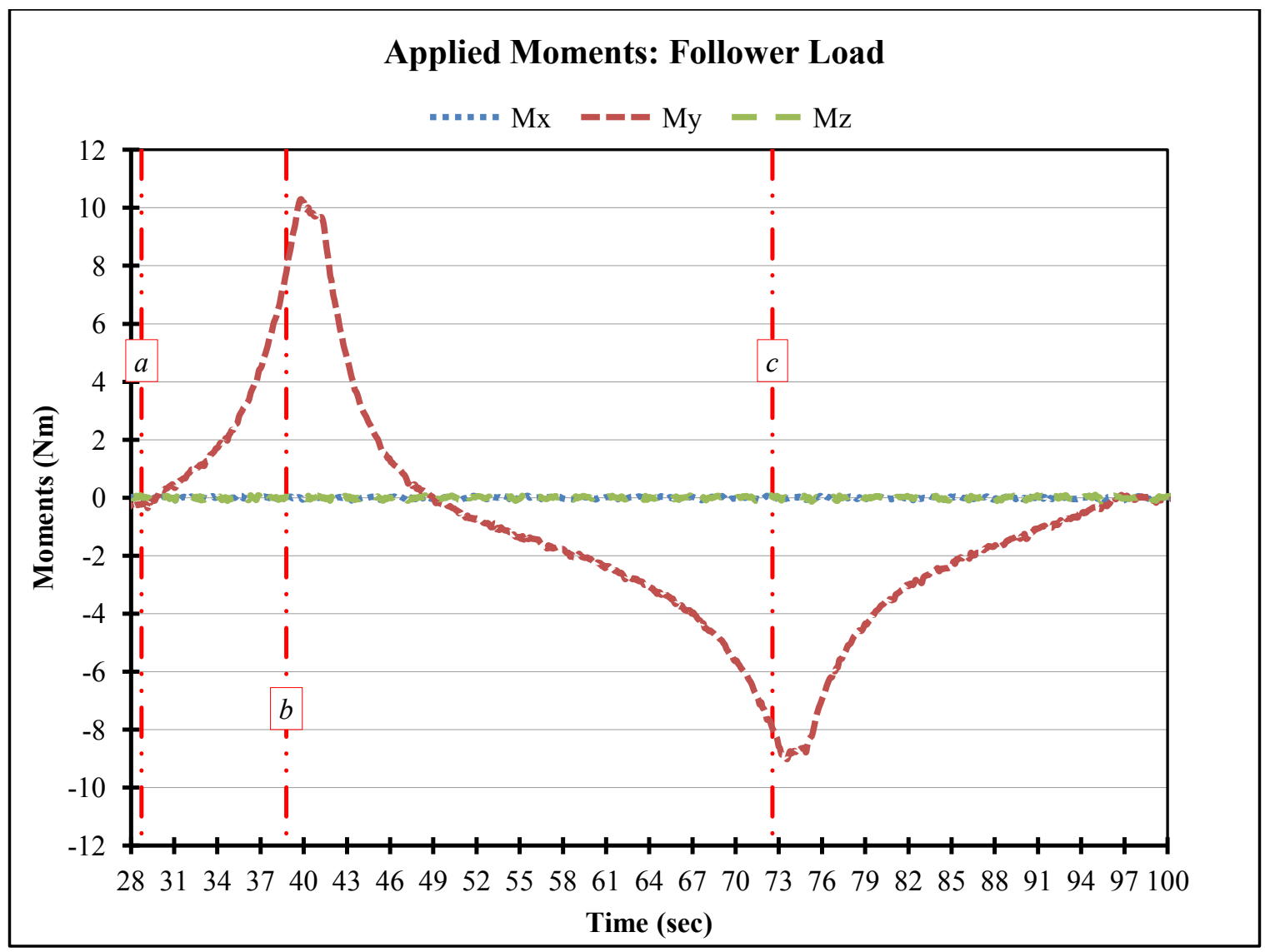

\section{Figure 3.5 Graphical representation of applied moments}

The lateral bending $\left(M_{x}\right)$ and CC torsion $\left(M_{z}\right)$ bending moments were commanded to 0 $\mathrm{Nm}$, therefore they are difficult to notice while flexion/extension $\left(M_{y}\right)$ is easily noted. RROM and displacement data were collected for analysis at instances of FL load without bending or $M_{y}=0 \mathrm{Nm}(a), 8 \mathrm{Nm}$ extension $(b)$, and $8 \mathrm{Nm}$ flexion $(c)$. The data were collected at a frequency of $10 \mathrm{~Hz}$. The sagittal moment overshoot was commanded to prevent any force error related to bending direction turnaround from affecting data analysis. 
Table 3.1 Mean commanded force TE

\begin{tabular}{lcccccc}
\hline Tracking Error Quantities & $\mathrm{F}_{\mathrm{x}}(\mathrm{N})$ & $\mathrm{F}_{\mathrm{y}}(\mathrm{N})$ & $\mathrm{F}_{\mathrm{z}}(\mathrm{N})$ & $\begin{array}{c}\mathrm{M}_{\mathrm{x}} \\
(\mathrm{Nm})\end{array}$ & $\begin{array}{c}\mathrm{M}_{\mathrm{z}} \\
(\mathrm{Nm})\end{array}$ & $\mathrm{F}_{\mathrm{R}}(\mathrm{N})$ \\
\hline Mean Tracking Error & 0.8 & 0.6 & 1.2 & 0.03 & 0.03 & 1.3 \\
SD of Mean TE & \pm 0.4 & \pm 0.3 & \pm 0.6 & \pm 0.00 & \pm 0.00 & \pm 0.6 \\
Avg. SD of TE for Single Test & \pm 0.8 & \pm 0.4 & \pm 1.6 & \pm 0.02 & \pm 0.02 & \pm 1.6 \\
\hline
\end{tabular}

The first row is the mean absolute value of the force TE at every data collection instance for all specimens. The second row is the SD of the means from individual specimen. The third row is the average SD of an individual specimen's mean absolute value of the force $\mathrm{TE}$ at every data collection instance. These data represent average instantaneous TEs in the system during testing and are subject to the effects of noise and system controllability.

Table 3.2 Mean anatomical point translation in sagittal plane

\begin{tabular}{lcc}
\hline Analysis Point & $\mathrm{X}_{\mathrm{avg}}(\mathrm{SD}) \mathrm{mm}$ & $\mathrm{Z}_{\mathrm{avg}}(\mathrm{SD}) \mathrm{mm}$ \\
\hline $0 \mathrm{Nm}$ No-Bend $(a)$ & $-0.2( \pm 0.3)$ & $-0.6( \pm 0.2)$ \\
$8 \mathrm{Nm}$ Extension $(b)$ & $-1.0( \pm 0.6)$ & $0.6( \pm 0.4)$ \\
$8 \mathrm{Nm}$ Flexion $(c)$ & $0.9( \pm 0.8)$ & $-2.5( \pm 0.9)$ \\
\hline
\end{tabular}

The average APTs noted in Figure 3.2 as tracked in the coordinate system defined by the mid-plane of the disc while the MSU is under no force or moment load. The x-axis is parallel to the sagittal mid-plane of the disc and the z-axis is normal to the mid-plane. The origin is determined by the location of the anatomical tracking point in the unloaded position. The three data analysis points $(a, b$, and $c)$ match up with the points mentioned in Figures 3.3 and 3.4. 


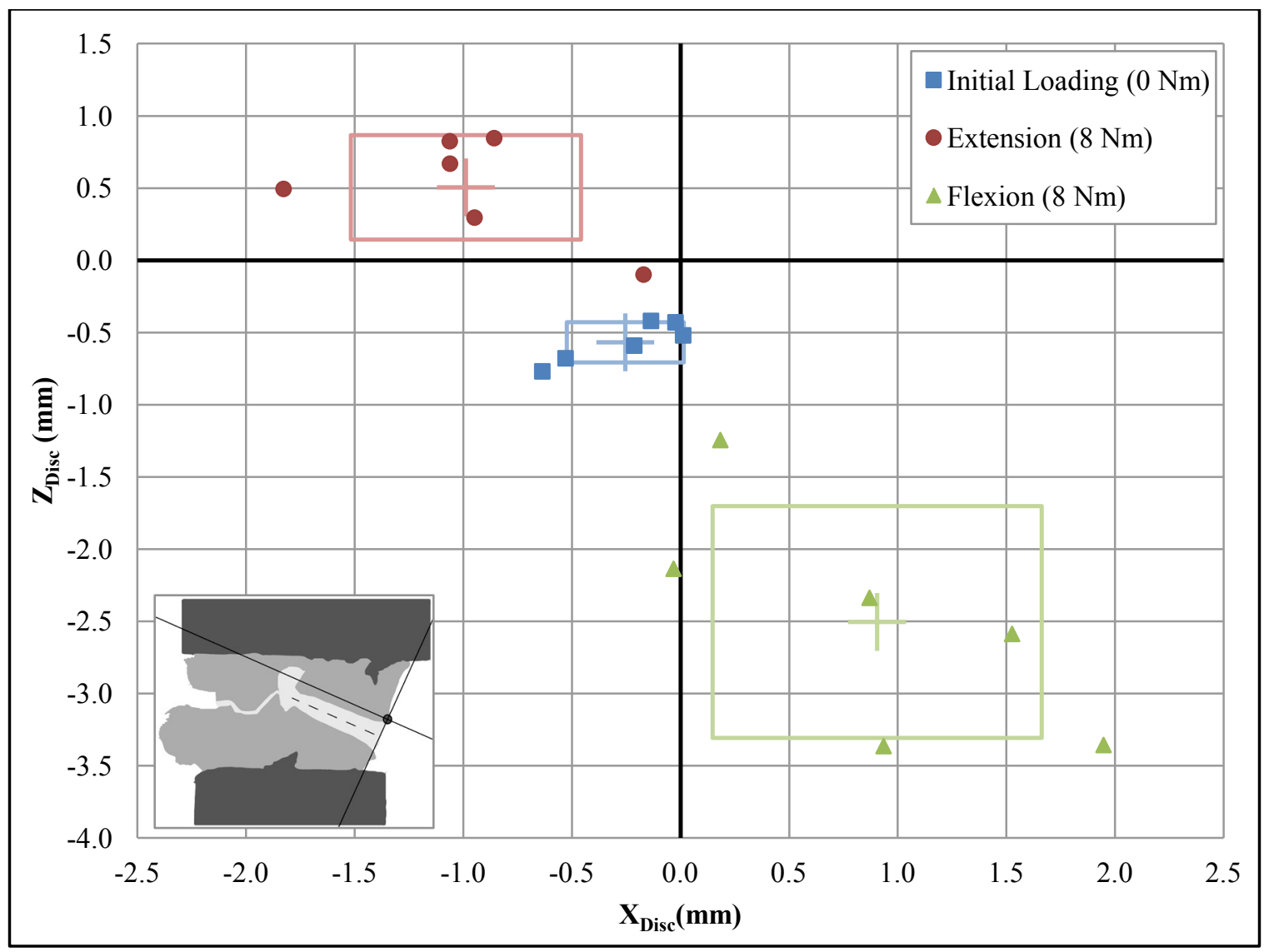

Figure 3.6 Anatomical point translation as result of loading and bending

The data displayed are the APTs noted in Figure 3.2 in the $\beta$ GCS. The origin is determined by the location of that point in the unloaded position. The crosses denote the averages and the boxes represent \pm 1 SD in both axes respectively. 


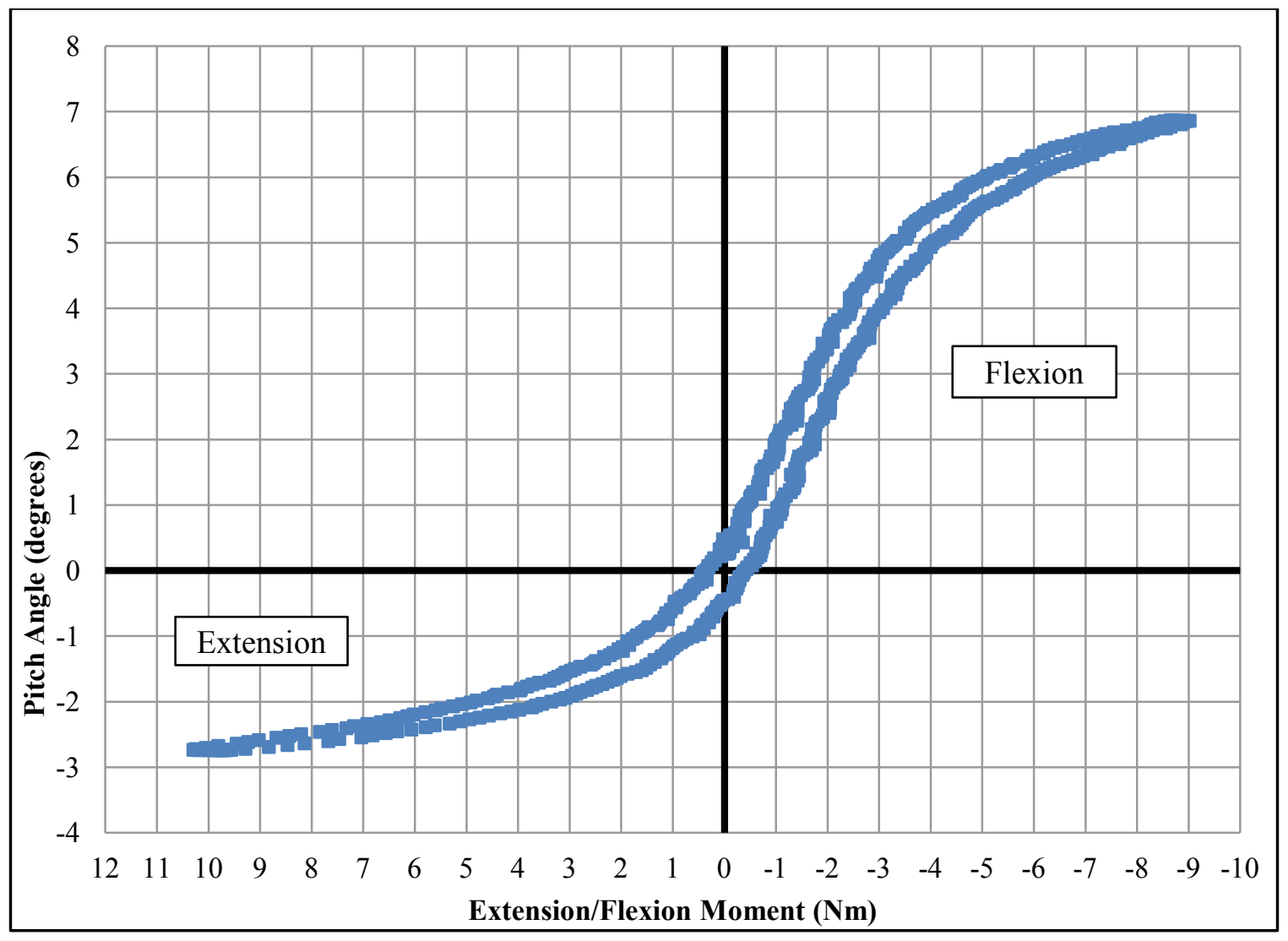

Figure 3.7 Rotational motion with respect to applied flexion/extension moments

$400 \mathrm{~N}$ ideal FL vector applied during $\pm 8 \mathrm{Nm}$ flexion/extension test. The typical flexion/extension flexibility graph displays path-dependent hysteresis as well as the typical NZ that is qualitatively defined by the two heels. This graph is meant purely for qualitative evaluation and comment, not data analysis. 


\subsection{Discussion}

One objective of this paper is to introduce a methodology for applying a dynamic force vector by robotically simulating an ideal FL protocol, during which a force of constant magnitude but dynamic direction is prescribed. Properties relating to robotic performance and tissue behavior were investigated: TEs, RROM, angular flexibility, and APT.

Due to the novelty of our system a limited amount of comparable data exists in the literature. ${ }^{24,44,45}$ Because of the direct load-feedback controlled nature of the platform, the load-control system clocks at $4.4 \mathrm{kHz}$, remarkably fast compared to other published systems. ${ }^{44}$ The average TEs for the zero-commanded axes (lateral shear, lateral bending, and $\mathrm{CC}$ rotation) were $0 \mathrm{~N}(2.12 \mathrm{~N} \mathrm{SD})$ and $0 \mathrm{Nm}(0.08 \mathrm{Nm} \& 0.10 \mathrm{Nm} \mathrm{SD})$, respectively as seen in Table 3.1. More notable TEs occurred in the axes bearing nonzero force commands (AP shear, compression, \& calculated $F_{R}$ ) however the TEs are negligible in the usual case $(<0.5 \%)$ and small in worst cases $(<5 \%)$ when compared to the commanded force targets. Our TEs are beyond satisfactory when compared to "unconstrained" axes TEs in robotic biomechanical testing literature: $\pm 25 \mathrm{~N}$ and $\pm 10 \mathrm{Nm}^{45}, \pm 6 \mathrm{~N}$ and $\pm 1 \mathrm{Nm}^{24}$, and $<1 \mathrm{~N}$ and $<0.05 \mathrm{Nm}$ RMS error ${ }^{44}$. Typically non-robotic test platforms do not present TEs for comparison.

The ideal FL as applied to a single MSU is applied normal to the midline of the disc. Numerous investigations have been conducted regarding the effects of variations in load vector magnitude, direction, location and path on flexibility and RROM, however the mechanisms for load application are similar: guided cables which direct the gravitational force on a deadweight. $6,7,8,9,12,20$

Several limitations plague this mechanism. Cable guides cause the loading vector to be oblique to the mid-plane of the disc during bending resulting. They also cause more artifact shear forces by gradually redirecting and misaligning tensile forces and leaving them to be resolved by the disc. Cable-driven testing apparatuses are also often used to apply static or quasi-static, non-physiologic motion profiles resulting in ligamentous relaxation. ${ }^{6,9}$ In answer to these limitations, the methodologies developed in this study provide solutions. Forgoing cables or guides, the platform maintained the direction of the load vector normal to the disc mid-plane during contiguous bending motion creating no artifact shear forces and mimicking physiologic bending motion.

The RROM results observed in this study were consistent with data from conventionally applied FL found in literature. ${ }^{6,9,12}$ And although the moment-angle graphs representing angular flexibility were not quantitatively evaluated, typical hysteresis was observed along with the characteristic heels separating the NZ from the "elastic zone.", ${ }^{21,52}$ The literature lacks sufficient APT data for comparison. It is therefore assumed that because observed RROM and flexibility data agrees with literature the translational data, though unparalleled, is acceptable as well. 
The system as it was used in this endeavor did not operate without compromises. Force TEs were greatest at flexion/extension direction reversals therefore slight moment overshoot was commanded to ensure that analyzed data were not affected heavily by TE. Also literature suggests that the location of the balance point from which reactive forces originate within the tissue changes under bending. ${ }^{6,9,40}$ The FRF transform used to negate moments caused by force couples in this study remained stationary with respect to the FMS. This however is not a limitation of the platform, but rather a simplification of protocol for the sake of preliminary investigation. Due to the dispensing of cables and guides, the robotic simulation of ideal FL is at this time relegated to single segments, a limitation born in exchange for non-sagittal expansion potential.

In light of a few compromises success and still greater potential exists. Future work involves making a load vector dynamic in direction as well as magnitude. Also, the aforementioned compromises are not limitations of the platform: FRF transforms have the potential to be mobile and the application of adaptive robotic performance parameters may result in better robotic performance. However, testing was achieved well within acceptable bending rates while being contiguous, without utilizing the platform's adaptive capabilities, and without a dynamic model of the specimen. Conclusively, a methodology for applying a dynamic load vector in coordination with bending was successfully developed. Simulation in non-sagittal planes is on the horizon. 


\section{CHAPTER 4. EFFECT OF LOAD ON KINEMATIC BEHAVIOR OF HUMAN LUMBAR SPINE DURING FLEXION-EXTENSION: COMPARISON OF ROBOTICALLY APPLIED PURE MOMENT, IDEAL FOLLOWER LOAD, AND NOVEL TRUNK WEIGHT PROTOCOLS}

\subsection{Introduction}

Spinal biomechanics investigators have used many different platforms to implement various in vitro loading protocols on cadaveric tissue in order to better understand the spine's natural kinematic behavior and the effects of myriad spinal instrumentation and corrective surgeries. Very seldom in the literature is there thorough comparison between different protocols or different testing platforms. And when comparisons are published, the metrics used to define kinematic characteristics are limited and potentially ineffective at identifying differences in the biomechanics observed during various protocols applied by various platforms, resulting in contradictions in the literature. $6,7,14,16,18,20,24,25,31,36,43,44,51,52,53,54,55$

Two current standard protocols for biomechanical spine testing are the PM and the FL protocols. ${ }^{13,14,15,16,17,18,19,20}$ The PM protocol ideally applies single plane bending and no other forces while the FL protocol ideally applies compressive forces normal to IVDs during single plane bending. ${ }^{12}$ Platforms use various mechanisms to apply prescribed loads: cables and pulleys ${ }^{56,57}$, or controller-driven actuators orthogonally nested as a gimbal, ${ }^{10,37}$ combined in series, ${ }^{24,25,58}$ or in parallel as a hexapod ${ }^{44,45}$ and almost all incorporate a multi-axis FMS and some form of a contact- or non-contact position tracking measurement system. ${ }^{59}$ The metrics based on data from differing platforms used to characterize kinematic response to applied loads are primarily angular rotation and flexibility which are subject to various levels of limitation and error determined by platform capabilities. ${ }^{20}$

Certain protocols and platforms are more prone to specific limitations than others. For instance, the PM protocol ideally involves the application of a single moment while all forces and other moments are constant at $0 \mathrm{~N}$ and $0 \mathrm{Nm}$, respectively. The controllability of certain platforms can result in the application of unwanted or "artifact" forces and moments during a test. ${ }^{45}$ Similar artifact forces and moments plague the application of a FL where artifact shear forces can be found in the unaccounted for loads on the disc. ${ }^{6}$ Furthermore, with regard to the conventional application of an FL protocol, the cables and guide conformation limit the application of the protocol to bending in the sagittal plane, although sometimes laboratories misapply this load. ${ }^{60}$ These limitations however do not encompass the plethora limitations and errors involved with various platforms and protocol applications. Nonetheless there exist numerous different platforms for applying biomechanical protocols.

One of the primary inspirations for this chaos of engineering is the question, "Does load affect the spine?" Panjabi et al. $(1977)^{21}$ found that load increased lateral and sagittal angular flexibility. However Miller and Skogland (1980) ${ }^{61}$ and Janevic et al. 
$(1991)^{62}$ found that when platform adjustments were made load decreased lateral and sagittal angular flexibility. Still further, Cripton et al. (2000) ${ }^{6}$ found that the load-spine kinematic relationship is dependent upon unintended "artifact" moments and shear while Patwardhan et al. (2003) ${ }^{9}$ concluded that load magnitude affects flexibility as well as RROM. The literature suggests that load affects the spine but the exactitude of the effect is suspect when considering the various protocols and method adjustments used to obtain quantitative evidence that is debatable at best. ${ }^{6,9,21,61,62}$

The literature is not entirely inconclusive but the fidelity of its conclusions provides room for improvement. For example numerous observations in literature are founded on the extrapolation of ideas (like the ideal FL) into protocol methodologies outside the initial domain of that particular idea's intended use (the sagittal plane). ${ }^{60,63}$ The literature has also historically been fixed on particular metrics for biomechanical evaluation. Unfortunately the primary metrics have offered little to no statistically significant differences in biomechanical protocol and platform comparisons when intuitive and sometimes observable differences are apparent. There is also growing concern for how shear may affect kinematic response due to the fact that it is present in vivo. ${ }^{2}$ To the author's knowledge, most published protocols attempt to either minimize forces or mimic ideal FL, which calls for only compression. A gap in the literature exists for investigation into the effect of intentional shear in combination with compression on spinal kinematics.

The objective of this study was to quantitatively compare two standard protocols, PM and the ideal FL and a trunk weight (TW) protocol involving the intentional application of shear in combination with compression by their effects on the kinematics of a single L4-L5 spinal MSU. The spinal kinematic characterizing metrics used to compare the protocols were flexion/extension RROM and APT, a quantity novel in its use for comparison. It was hypothesized that additional compressive and shear load would decrease $\mathrm{RROM}^{9}$ and that shear load will produce statistically significant differences in displacement.

\subsection{Materials and Methods}

Six fresh-frozen human cadaveric L4-L5 lumbar MSUs (male, mean age $44 \pm 8.9$ years SD) were radiographically pre-screened for degenerative disc disease symptoms, harvested and cleaned of excessive soft tissue. The segments were potted in bismuth alloy (Cerrobend ${ }^{\mathrm{TM}}$ ) in specimen-specific, natural lordotic alignment referenced to the assumed horizontal cranial endplate of L1 as in Figure 3.1. The specimens were caudally fixed within the platform and cranially attached to the Cartesian manipulator.

The testing platform was comprised of a custom-built, Cartesian, RTLC Gantry system with six fully controlled DOF and a six-axis FMS (Model 45E15A4-I63-AF JR3 Inc., Woodland, CA). Three harmonic drive motors comprising a roll-pitch-yaw (lateral bending, flexion-extension, axial rotation) gimbal assembly were suspended in series from three servo-actuated orthogonally oriented $\mathrm{x}-(\mathrm{AP}), \mathrm{y}$-(lateral), and $\mathrm{z}-(\mathrm{CC})$ 
translational axes which, collectively comprised a single Cartesian manipulator. ${ }^{10}$ The FMS was rigidly mounted between the gimbal and cranial end of the specimen.

All axes were controlled via a PID gain controller (UMAC Delta Tau Data Systems Inc., Chatsworth, CA) implicating custom coded programs, which prescribed targeted loading scenarios. Each axis could operate utilizing position- or force-feedback but regardless of that mechanism all movements were coordinated. A custom calibration algorithm was employed in real-time to correct for changes in FMS readings due to gravitational forces on attached pots and fixtures such that the platform directly controlled loads applied to the specimen.

Specimens were mounted in the testing platform and manipulated to an unloaded state that delineated neutral angular orientation. The tissue was preconditioned by applying a flexion-extension PM protocol (all forces and non-sagittal moments commanded to $0 \mathrm{~N}$ and $0 \mathrm{Nm}$, respectively) to an $8 \mathrm{Nm}$ end-limit three times. The flexion/extension motor was driven in position-control to ensure a constant bending rate of $0.35 \%$ sec during all tests while all other motors operated in load-control. To ensure applied forces did not build up initial moment artifacts a $400 \mathrm{~N}$ resultant load vector was applied normal to the disc by sequentially applying $F_{z}$ and $F_{x}$ component forces while constraining flexion-extension rotation. The magnitudes of those component forces were determined by trigonometric equations which will be elaborated on in the following paragraphs. Observed sagittal moment values in this state were due to offset net reactive specimen forces with respect to the FMS FRF. The FRF was transformed in the $F M S-C S_{X}$ and $F M S-C S_{Z}$ directions to null these values and subsequent moment values were determined with respect to the newly located reference frame for all tests.

Two unique loading scenarios were simulated: the ideal FL and the novel TW load. The development of the FL was in response to a problem exhibited by in vitro testing of multi-segment spine specimen. Compressive magnitudes of in vivo magnitudes would cause the spine to collapse or bend over. Patwardhan et. al (1999) ${ }^{12}$ addressed this issue by guiding the compressive force tangentially along the lumbar curvature of the spine through the theoretical ICR of each disc. Subsequently the ideal FL would apply a force normal to the midline of the disc with minimal shear forces. ${ }^{9}$ When regarding the coordinate systems established within this research, an ideal FL would be a combination of $F_{x}$ and $F_{z}$ that resulted in a force vector with a direction normal to the midline of the disc at all times.

The FL has been conventionally applied by connecting deadweight to the cranial body of specimen with cables. The cables were then run through eyelets that are connected to the lateral sides of the vertebral bodies of the specimen. The vertical location of those eyelets was typically in the middle of each body while the horizontal location for each eyelet was dependent on specimen specific radiographic images used to approximate the ICR. The locations of each eyelet would dictate the "path" of the FL. Eventually the methodology for path creation evolved, incorporating empirical path optimization. The load is applied to the cable and the horizontal locations of the eyelets were altered until minimal changes in flexion/extension or lordosis as result of the 
deadweight were read by non-contact markers on all bodies. This mechanically meant that when the path was determined by the optimal location of the eyelets (primarily variable horizontally), the deadweight would be applying the smallest net moment to the specimen, while the specimen was in a specific posture. 9,12

This methodology, although commonly accepted, presents a few limitations. One is that the location of the eyelets remains constant through bending. Patwardhan et. al (1999) observed that optimization of path is posture dependent or in other words, the optimal horizontal location of the eyelets changes. This means that the cables apply unwanted or "artifact" moments during flexion and extension. Also the optimal path minimizes any artifact moments from the deadweight that would cause change in flexion/extension or lordosis, but there are still shear forces applied to each disc resulting from the redirection of the tensile forces in the cables. Although the compressive FL theoretically minimizes shear forces at each disc, the practical application of the protocol is than ideal contrary to the force vectors' supposed alignments being normal to the midline of each disc. These are but a few limitations of the conventional application of the FL protocol.

In this research some key features of the ideal FL were used to found the simulation of the ideal FL. One of the primary focuses was that the ideal FL vector would be applied perpendicularly to the mid-plane of the disc as depicted in Figure 3.2. ${ }^{9}$ During sagittal bending the mid-plane of the disc rotates half as much as the cranial body. This required the load vector to change direction with respect to the FMS which was providing control feedback for the motors responsible for maintaining a constant resultant magnitude. ${ }^{9}$ Therefore, at $0.2^{\circ}$ increments of sagittal rotation, AP and CC forces were commanded by Equation 3.1 and 3.2 where B is the angle of the top body and $\beta$ is the angle between the mid-plane of the disc and the top pot. A load magnitude of $400 \mathrm{~N}$ was chosen for simulation ${ }^{48,49,50}$ while the $\mathrm{Y}$-axis, lateral and axial rotation axes targeted $0 \mathrm{~N}$ and $0 \mathrm{Nm}$, respectively. All of these forces were read and consequently applied at the point of the FRF transform determined for each specimen upon mounting.

The TW load is similar to FL in its requirement of a dynamic vector of constant magnitude, however it is dissimilar because of its disc mid-plane shear component. Due to the in vivo angulation of the L4-L5 disc, the weight of the trunk applies a shear force across the disc as displayed in Figure 2.6. Therefore the direction of TW force vector was intended to be static with respect to the GCS in efforts to simulate gravity. Instead of remaining normal to the mid-plane of the disc the vector remains vertically oriented within the GCS as in Figure 4.1. At $0.2^{\circ}$ increments of sagittal rotation commanded FMS forces were thus programmed using Equations 4.1 and 4.2:

$$
\begin{aligned}
& F_{x}=400 \sin (B) \\
& F_{z}=400 \cos (B)
\end{aligned}
$$

Resultant load magnitudes were maintained from the FL protocol. 


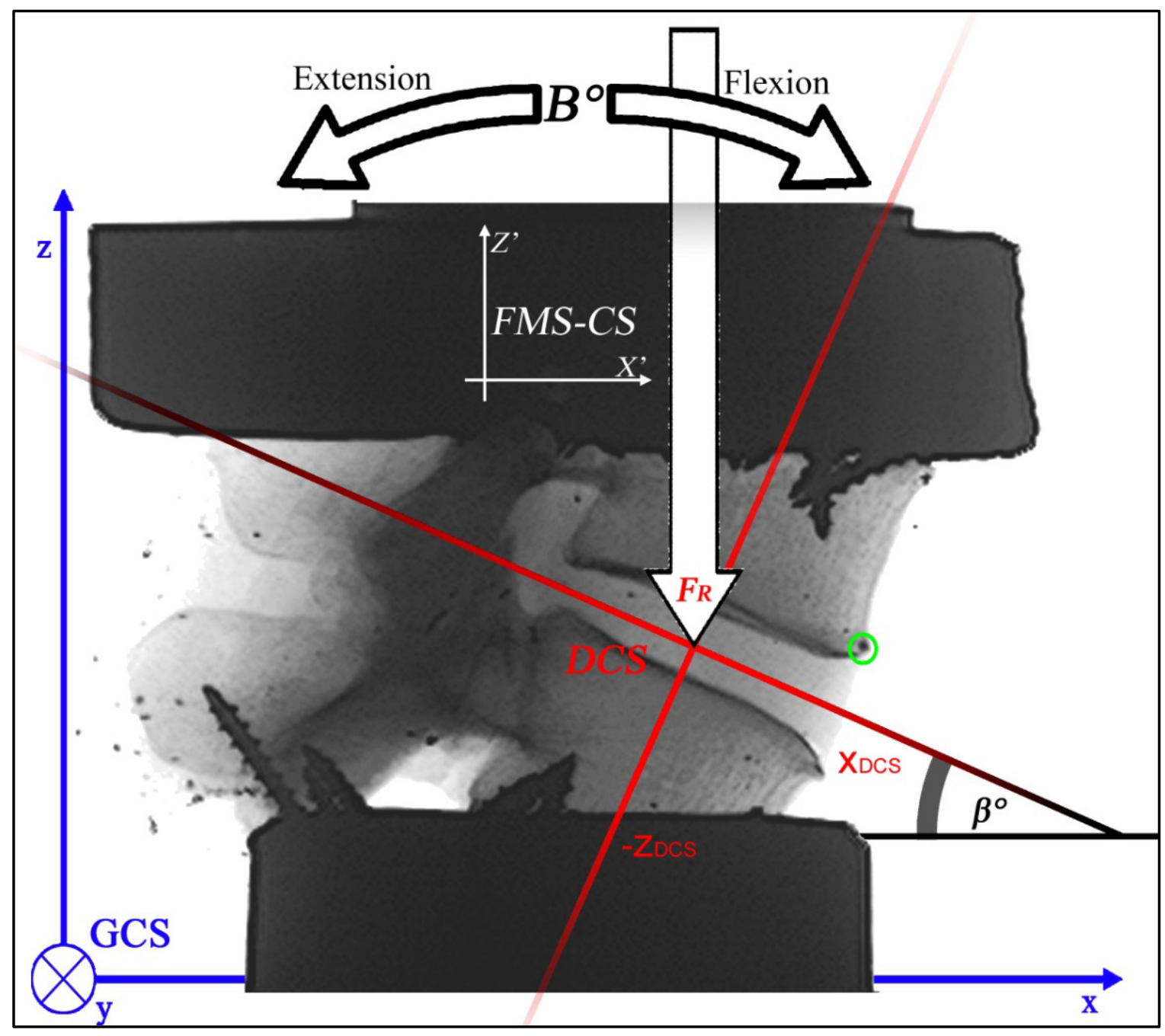

\section{Figure 4.1 Ideal TW application to lumbar MSU}

The component force $\left(F_{x}\right.$ and $\left.F_{z}\right)$ as seen in Figure 3.2 are not present here because $F_{R}=$ $F_{z}$ and $F_{x}=0 \mathrm{~N}$. However they are similarly dependent on the magnitude of the commanded resultant force $\left(\mathrm{F}_{\mathrm{R}}\right)$ and the mobile flexion/extension angle $(B)$ but not the specimen specific sagittal potting angle $(\beta)$. Based on Equations 4.1 and 4.2 the resultant force remains normal or vertical to the horizontal plane of the GCS (blue). For example, as L4, the FMS and the Cartesian manipulator flex forward the commanded $F_{x}(0 \mathrm{~N}$ here $)$ will increase in positive magnitude while $F_{z}$ decreases. Also note the anatomic tracking point (green circle) used to define the displacement metric. The FMS-CS is in white and the DCS is in red. 
The loaded specimens were flexed and extended to $8 \mathrm{Nm}$ three times then offloaded for a final PM bending protocol of three $8 \mathrm{Nm}$ flexion-extension cycles. RROM data from the third preconditioning PM cycle and the third final PM cycle were informally inspected for differences greater than $0.2^{\circ}$. None were observed.

Rotational, translational, force, and moment data were recorded at $10 \mathrm{~Hz}$ for analysis from the third bending cycle of each test. Translational and rotational data along with fixturing and radiographic measurements were used to calculate the Cartesian displacement data of the anterior-most point of the superior vertebral body's inferior endplate as noted in Figure 4.1. RROM and Cartesian displacement were analyzed under no load, neutrally loaded, $8 \mathrm{Nm}$ flexed and $8 \mathrm{Nm}$ extended conditions. The observed $F_{x}$ and $F_{z}$ forces were combined to determine the resultant applied force for TE calculation.

The RROM data were used to quantify the differences between protocols by a commonly accepted method ${ }^{53}$ while the translational data offered another perspective. All data were tested for normality using the Pearson's Chi squared test: measured RROM and transformed anatomic position tracking displacements analyzed at the three specified analysis points, neutral, extension, and flexion. Repeated-measures analyses of variance were used to discern differences across protocols in RROM and translational data. Greenhouse-Geisser estimations of $\varepsilon$ were applied to the DOF where appropriate. For post hoc analysis paired t-tests compared protocols utilizing the Sidak correction to the level of significance $(\alpha)$ accounting for multiple comparisons. Average load TE was calculated to evaluate robotic performance.

\subsection{Results}

FMS values were used to calculate resultant force $\left(F_{R}\right)$ values and accompanying TEs. Examples of force and moment performance during a test of each protocol are exemplified in Figures 4.2 through 4.4. Average force and moment TEs for all protocols were below $2.0 \mathrm{~N}$ and $0.05 \mathrm{Nm}$, respectively. The mean resultant force $\left(F_{R}\right)$ TE during FL and TW protocols was less than $0.5 \%$ of the targeted value $(400 \mathrm{~N})$. Average SDs of TEs for single axes during individual tests were calculated and displayed in Table 4.1. Load-controlled axes were controlled within maximum envelopes of $\pm 5 \mathrm{~N}$ or $\pm 0.2 \mathrm{Nm}$ of their targets. Flexion/extension reversals caused greater TEs necessitating slight commanded sagittal bend overshoot to allow the analyzed results to bear minimal error as seen in Figures 4.2B through 4.4B.

It is important to note in the PM forces graph (Figure 4.2A) that the scatter present represents the combined effects of system noise, system controllability, and FMS resolution. The FMS is an analogue tool with a specific sensitivity which was used to calculate the resolutions of each of the 6 axes: $F_{x}$ and $F_{y}=0.06 \mathrm{~N}, F_{z}=0.11 \mathrm{~N}, M_{x}, M_{y}$, and $M_{z}=0.006 \mathrm{Nm}$. It is therefore safe to say that the controllability of the system is not adversely affected by the resolution of the FMS. 


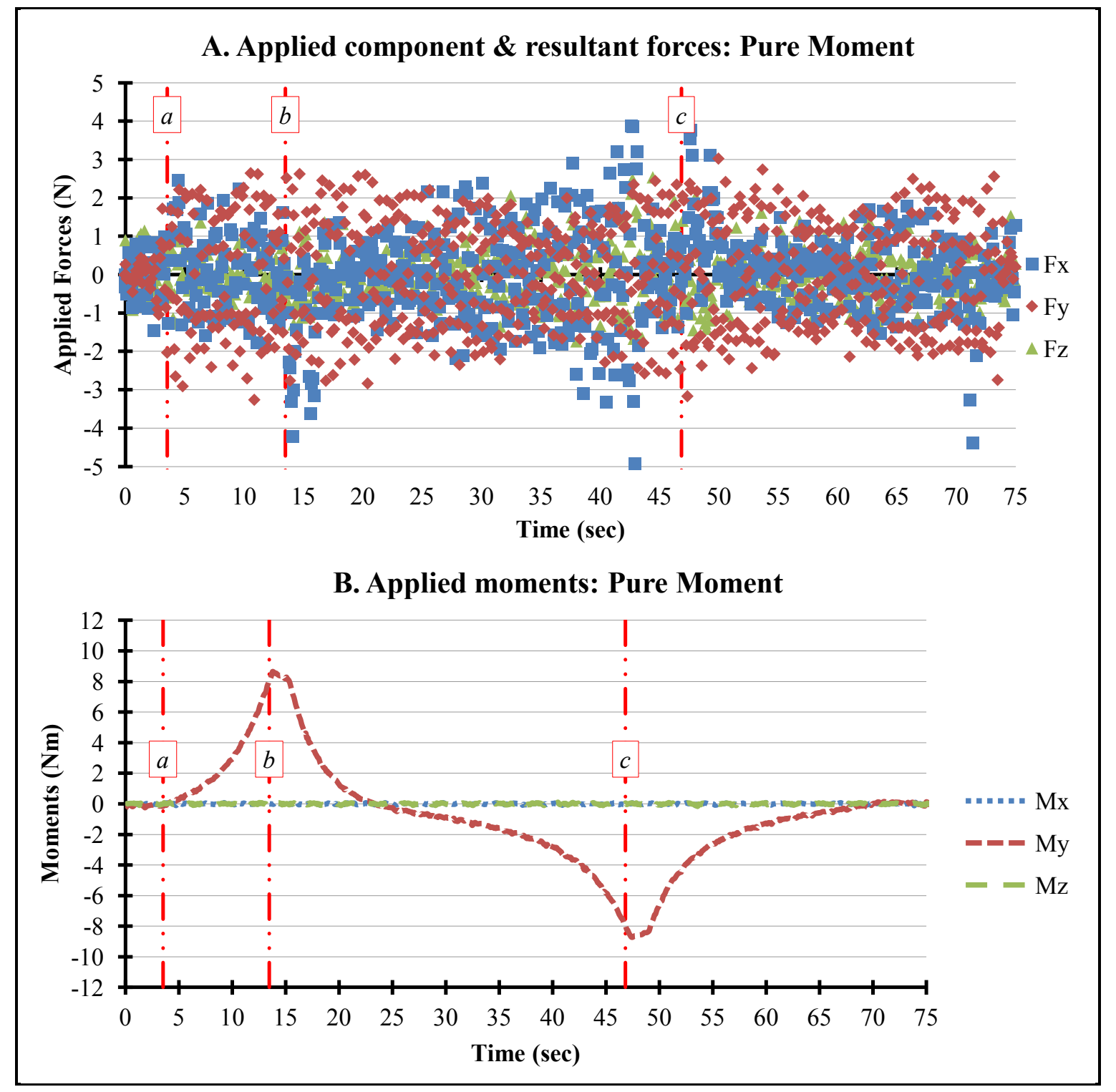

Figure 4.2 Graphical representation of applied forces and moments during PM protocol

The various forces (A) were commanded to $0 \mathrm{~N}$. The moments (B) are represented with the lateral bending $\left(M_{x}\right)$ and $\mathrm{CC}$ torsion $\left(M_{z}\right)$ moments commanded to $0 \mathrm{Nm}$. The flexion/extension moment $\left(M_{y}\right)$ is more easily noticed because it was not being commanded to $0 \mathrm{Nm}$. RROM and displacement data were collected for analysis at instances of no bending or $M_{y}=0 \mathrm{Nm}(a), 8 \mathrm{Nm}$ extension $(b)$, and $8 \mathrm{Nm}$ flexion $(c)$. The data was collected at a frequency of $10 \mathrm{~Hz}$. 


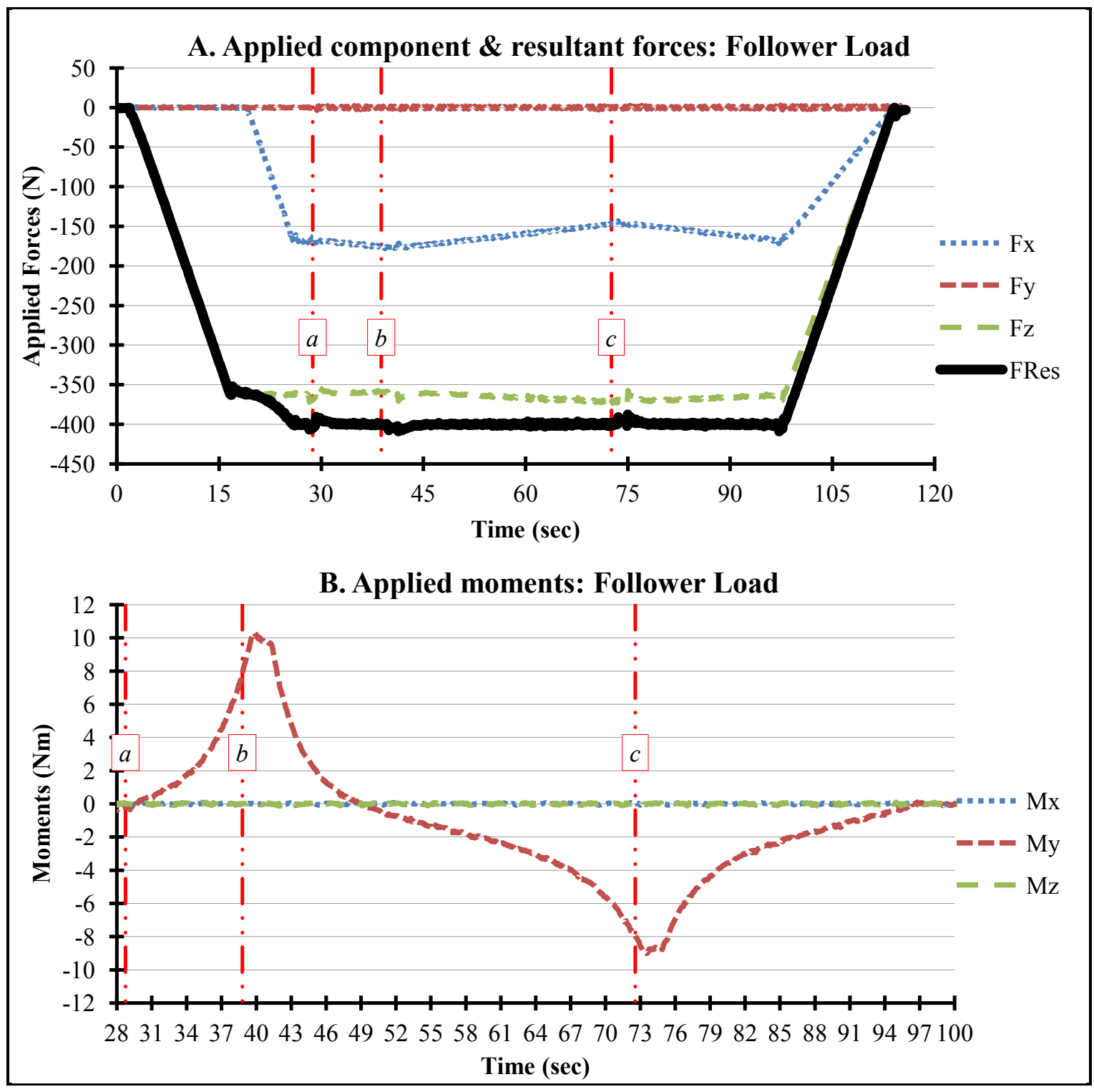

Figure 4.3 Graphical representation of applied forces and moments during FL protocol

Force $(\mathrm{A})$ in lateral shear $\left(F_{y}\right)$ and resultant force $\left(F_{R}\right)$ were commanded to constant values of $0 \mathrm{~N}$ and $400 \mathrm{~N}$, respectively. It would be potentially confusing, however, to define AP shear and $\mathrm{CC}$ compressive force as $\left(F_{x}\right.$ and $\left.F_{z}\right)$ because of the nature of their relationship involving the flexion/extension moment. They were respectively commanded by Equation 3.1 and 3.2.The moments (B) are represented below with the lateral bending $\left(M_{x}\right)$ and $\mathrm{CC}$ torsion $\left(M_{z}\right)$ moments commanded to $0 \mathrm{Nm}$. The flexion/extension moment $\left(M_{y}\right)$ is more easily noticed because it was not being commanded to $0 \mathrm{Nm}$. RROM and displacement data were collected for analysis at instances of no bending or $M_{y}=0 \mathrm{Nm}$ $(a), 8 \mathrm{Nm}$ extension $(b)$, and $8 \mathrm{Nm}$ flexion $(c)$. The data was collected at a frequency of $10 \mathrm{~Hz}$. 


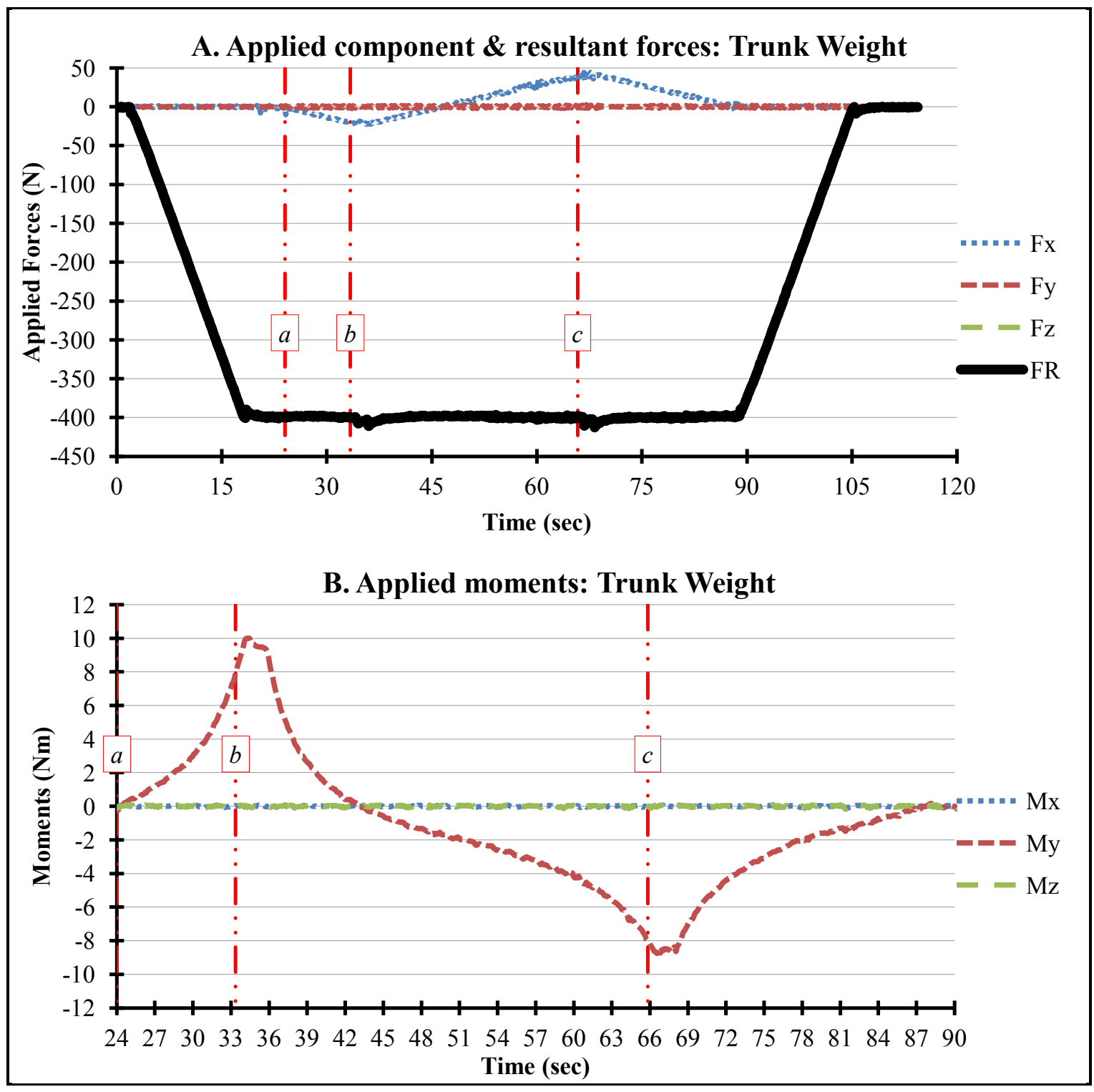

Figure 4.4 Graphical representation of applied forces and moments during TW protocol

Force $(\mathrm{A})$ in lateral shear $\left(F_{y}\right)$ and resultant force $\left(F_{R}\right)$ were commanded to constant values of $0 \mathrm{~N}$ and $400 \mathrm{~N}$, respectively. It would be potentially confusing, however, to define AP shear and $\mathrm{CC}$ compressive force as $\left(F_{x}\right.$ and $\left.F_{z}\right)$ because of the nature of their relationship involving the flexion/extension moment. They were respectively commanded by Equations 4.1 and 4.2. The moments (B) are represented below with the lateral bending $\left(M_{x}\right)$ and $\mathrm{CC}$ torsion $\left(M_{z}\right)$ moments commanded to $0 \mathrm{Nm}$. The flexion/extension moment $\left(M_{y}\right)$ is more easily noticed because it was not being commanded to $0 \mathrm{Nm}$. RROM and displacement data were collected for analysis at instances of no bending or $M_{y}=0 \mathrm{Nm}(a), 8 \mathrm{Nm}$ extension $(b)$, and $8 \mathrm{Nm}$ flexion (c). The data was collected at a frequency of $10 \mathrm{~Hz}$. 
Table 4.1 Mean commanded force tracking error

\begin{tabular}{lcccccc}
\hline Mean Tracking Error & $\mathrm{F}_{\mathrm{x}}$ & $\mathrm{F}_{\mathrm{y}}$ & $\mathrm{F}_{\mathrm{z}}$ & $\mathrm{M}_{\mathrm{x}}$ & $\mathrm{M}_{\mathrm{y}}$ & $\mathrm{F}_{\mathrm{R}}$ \\
& $(\mathrm{N})$ & $(\mathrm{N})$ & $(\mathrm{N})$ & $(\mathrm{Nm})$ & $(\mathrm{Nm})$ & $(\mathrm{N})$ \\
\hline Pure Moment & 0.6 & 0.7 & 0.4 & 0.03 & 0.03 & $\mathrm{NA}$ \\
& $(0.2)$ & $(0.3)$ & $(0.1)$ & $(0.00)$ & $(0.00)$ & $\mathrm{NA}$ \\
Follower Load & $((0.6))$ & $((0.5))$ & $((0.4))$ & $((0.02))$ & $((0.02))$ & $\mathrm{NA}$ \\
& 0.8 & 0.6 & 1.2 & 0.03 & 0.03 & 1.3 \\
& $(0.4)$ & $(0.3)$ & $(0.6)$ & $(0.00)$ & $(0.00)$ & $(0.6)$ \\
Trunk Weight & $((0.8))$ & $((0.4))$ & $((1.6))$ & $((0.02))$ & $((0.02))$ & $((1.6))$ \\
& 1.0 & 0.8 & 1.4 & 0.03 & 0.03 & 1.4 \\
& $(0.2)$ & $(0.2)$ & $(0.5)$ & $(0.00)$ & $(0.00)$ & $(0.4)$ \\
& $((1.0))$ & $((0.5))$ & $((1.6))$ & $((0.02))$ & $((0.03))$ & $((1.4))$ \\
\hline
\end{tabular}

The first value is the mean absolute value of the force TE at every data collection instance for all specimens. The second value marked by single parentheses is the SD of the means from individual specimen. The third value marked by double parentheses is the average SD of an individual specimen's mean absolute value of the force TE at every data collection instance. The measurements taken directly from the FMS $\left(F_{x}, F_{y}, F_{z}\right.$, $M_{x}$, and $M_{y}$ ) were compared to respective targets in the FMS-CS while $F_{R}$ was compared to a $400 \mathrm{~N}$ target in the DCS. These data represent average instantaneous TEs in the system during testing and are subject to the effects of noise and system controllability. 
RROM was normalized to the sagittal bend angle where no moment was present. No statistically significant difference between protocols was evident. Moment-angle flexibility graphs demonstrate typical hysteresis but no remarkable trends in flexibility variation were found. Sagittal plane displacement due to loading and bending was determined by tracking the anterior corner of the inferior endplate of L4 in the $\beta$ GCS. Graphical representation of APT data in Figure 4.5 shows mean and SD translational values resulting from initial loading, extension, and flexion. Mean independent axis translations were compared for statistical significance between protocols. Statistically significant differences are noted in Table 4.2.

\subsection{Discussion}

The objective of this study was to quantitatively compare three spine testing protocols using a RTLC testing apparatus. A novel testing protocol was introduced. The new protocol incorporating intentional shear was compared to the current standard protocols by the RROM and APT.

Results of this study are limited to male spines with mild degenerative changes. The specimen used in this study came from a relatively young age group and showed no radiographic signs of degenerative changes. Protocol sequence was not randomized and although the author noted no effects of the loading sequence.

Robotic application of these protocols is new in biomechanics, specifically the methodology of RTLC of motors. Those laboratories that have developed non quasi-static load-control have employed platforms in the application of PM protocols, targeting $0 \mathrm{~N}$

and $0 \mathrm{Nm}$ for non-bending axes. ${ }^{24,44,52}$ In comparison with the literature, the present study TEs for the PM protocol are excellent. The TE values for the loaded protocols compete with and in some cases best the lowest TEs of other robotically applied PM protocols in the literature. ${ }^{24,44,45,52,58}$ Although a few other laboratories have employed robotics to apply biomechanics protocols with RTLC, ${ }^{44}$ none, to the author's knowledge, have used the capability to apply a load vector. Fewer still have had success comparable applying PM with acceptably low TEs. ${ }^{24,45,52,58}$ Also this system requires no complex model describing the control environment for operation. ${ }^{25}$

Combined flexion/extension RROM data observed in this study are consistent with those reported in literature. ${ }^{48,49,56,57,64}$ And although publications presenting separated flexion/extension RROM data are sparse and such reports often utilize subjective means for delineation, the data from this study are consistent with those values as well. A trend of decreasing RROM was observed when load was applied in FL and TW protocols as seen in Figure 4.6. Similarly this trend is exemplified by Cripton et al. $(2000)^{6}$ and Patwardhan et al. $(2003)^{9}$ both of whom concluded that angular decrease in RROM is dependent on the magnitude of the applied load. It is possible that $400 \mathrm{~N}$ was insufficient to illicit a statistically significant difference in RROM. The decrease in RROM is perhaps due to increase in internal disc pressure resulting in stiffer mechanics or the impingement of the articulating surfaces of the facets. ${ }^{4,12,35}$ 


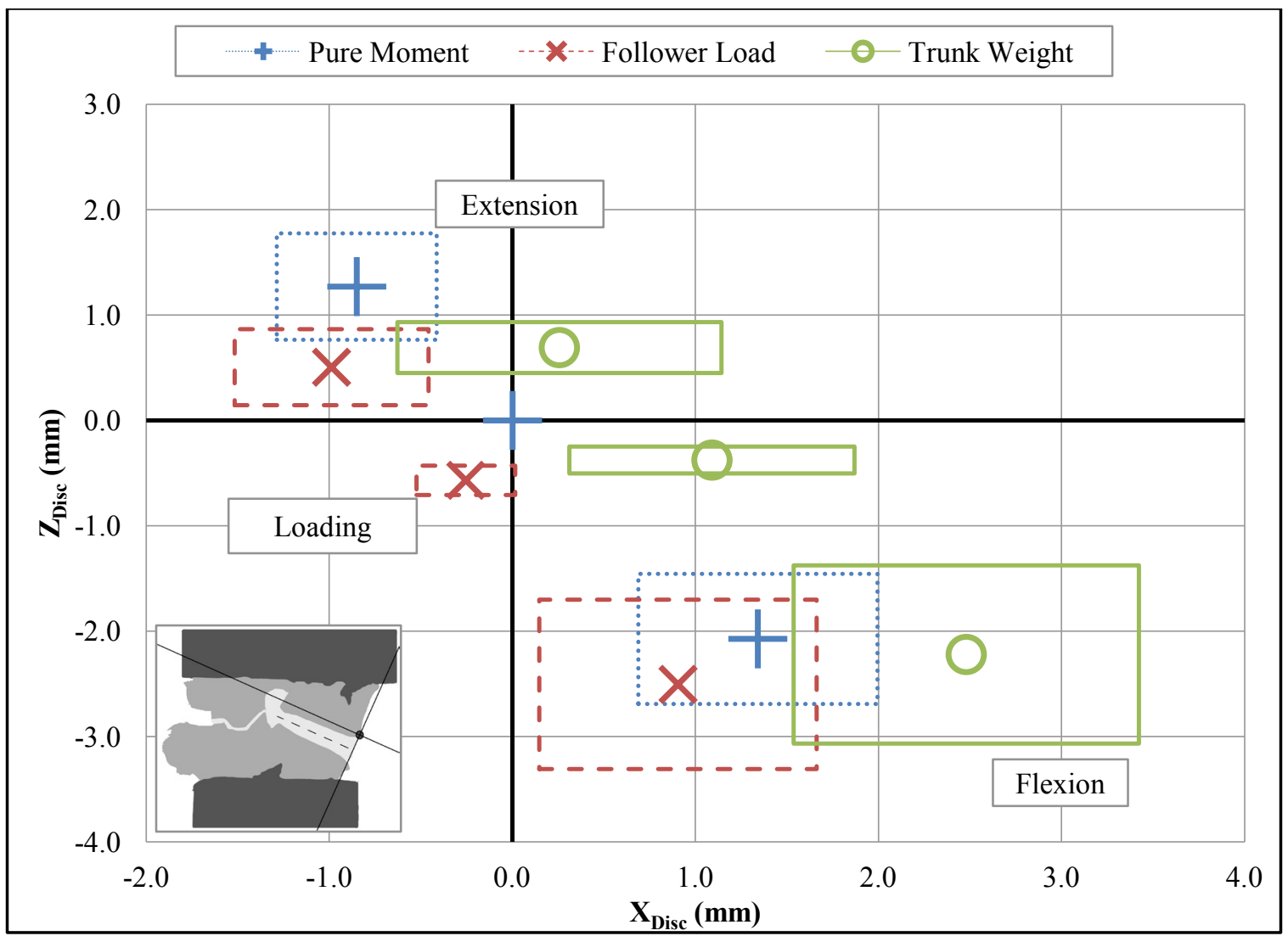

Figure 4.5 Mean anatomical point translation in different protocols

The data displayed are the APTs noted in Figure 3.2 in the coordinate system defined by the unloaded disc where the $\mathrm{x}$-axis is parallel to the disc and the $\mathrm{z}$-axis is normal to it. The origin is determined by the location of that point in the unloaded position. The bold data points denote the averages and the boxes represent \pm 1 SD in both axes respectively. Three general groupings of data points represent extension (top-left quadrant), loading (around origin), and flexion (bottom right quadrant).

Notice that although the loading of the specimen shows significant difference in translation between protocols that the flexion/extension translation is less significantly different suggesting a robust kinematic behavior of the joint during bending, performing similarly under load to its behavior under no load. 
Table 4.2 Mean anatomical point translation

\begin{tabular}{llccl}
\hline Analysis & Parameter & Mean $(\mathrm{mm})$ & $\mathrm{SD}(\mathrm{mm})$ & Statistical Significance \\
\hline Loading $_{1}$ & PM X $\left(\mathrm{a}_{\mathrm{i}}\right)$ & 0.0 & 0.0 & $\mathrm{c}_{1}: \mathrm{p}=0.046$ \\
& FL X $\left(\mathrm{b}_{\mathrm{i}}\right)$ & -0.2 & 0.3 & $\mathrm{c}_{1}: \mathrm{p}=0.016$ \\
& TW X $\left(\mathrm{c}_{\mathrm{i}}\right)$ & 1.1 & 0.7 & $\mathrm{a}_{1}, \mathrm{~b}_{1}$ \\
& PM Z $\left(\mathrm{d}_{\mathrm{i}}\right)$ & 0.0 & 0.0 & $\mathrm{e}_{1}: \mathrm{p}=0.003, \mathrm{f}_{1}: \mathrm{p}=0.012$ \\
& FL Z $\left(\mathrm{e}_{\mathrm{i}}\right)$ & -0.6 & 0.2 & $\mathrm{~d}_{1}$ \\
& TW Z $\left(\mathrm{f}_{\mathrm{i}}\right)$ & -0.4 & 0.2 & $\mathrm{~d}_{1}$ \\
Extension $_{2}$ & PM X $\left(\mathrm{a}_{\mathrm{i}}\right)$ & -0.8 & 0.4 & $\mathrm{c}_{2}: \mathrm{p}=0.058$ \\
& FL X $\left(\mathrm{b}_{\mathrm{i}}\right)$ & -1.0 & 0.6 & $\mathrm{c}_{2}: \mathrm{p}=0.003$ \\
& TW X $\left(\mathrm{c}_{\mathrm{i}}\right)$ & 0.1 & 0.9 & $\mathrm{a}_{2}, \mathrm{~b}_{2}$ \\
& PM Z $\left(\mathrm{d}_{\mathrm{i}}\right)$ & 1.2 & 0.5 & \\
& FL Z $\left(\mathrm{e}_{\mathrm{i}}\right)$ & 0.6 & 0.4 & \\
& TW Z $\left(\mathrm{f}_{\mathrm{i}}\right)$ & 0.7 & 0.3 & \\
& PM X $\left(\mathrm{a}_{\mathrm{i}}\right)$ & 1.3 & 0.7 & $\mathrm{c}_{3}: \mathrm{p}=0.020$ \\
& FL X $\left(\mathrm{b}_{\mathrm{i}}\right)$ & 0.9 & 0.8 & $\mathrm{c}_{3}: \mathrm{p}=0.004$ \\
& TW X $\left(\mathrm{c}_{\mathrm{i}}\right)$ & 2.5 & 0.9 & $\mathrm{a}_{3}, \mathrm{~b}_{3}$ \\
& PM Z $\left(\mathrm{d}_{\mathrm{i}}\right)$ & -2.0 & 0.7 & $\mathrm{e}_{3}: \mathrm{p}=0.035$ \\
& FL Z $\left(\mathrm{e}_{\mathrm{i}}\right)$ & -2.5 & 0.9 & $\mathrm{~d}_{3}$ \\
& TW Z $\left(\mathrm{f}_{\mathrm{i}}\right)$ & -2.3 & 0.8 & \\
\hline
\end{tabular}

The anatomic point being tracked is the anterior-most point of the inferior endplate of the top body denoted by Figure 3.2. The point is being tracked through the $\beta \mathrm{GCS}$. Statistical significance, when present, is described by noting the case between which there is a difference and the corresponding $p$-value. The null hypothesis was rejected and subsequently statistically significant differences were accepted at $p<0.05$. The right column lists the cases between which the pertinent row exhibits a statistically significant difference along with the associated $p$-value. Nearly significant differences were listed as well. 


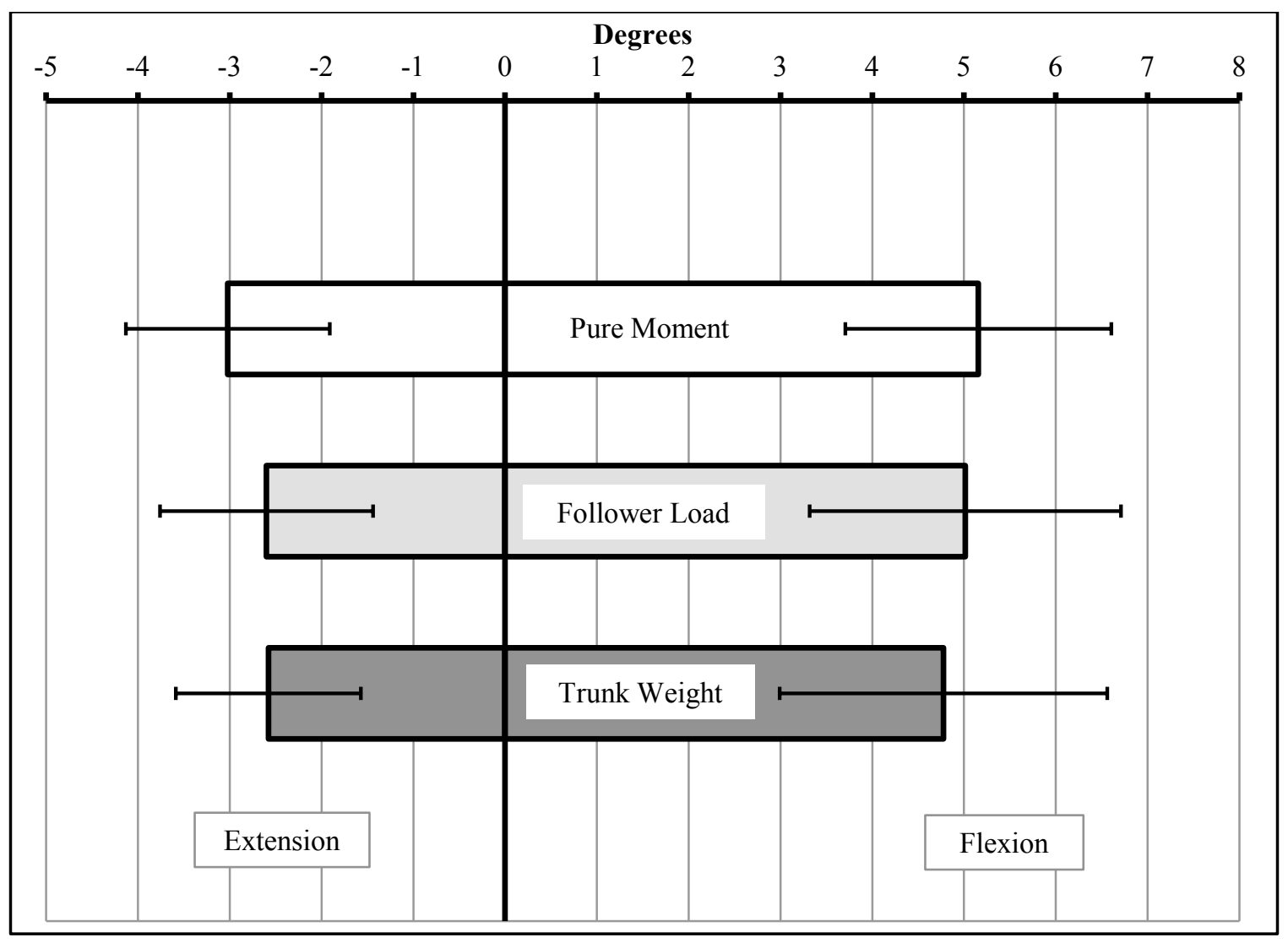

Figure 4.6 Mean flexion-extension RROM of different protocols

RROM was measured by analyzing the sagittal plane rotation at $8 \mathrm{Nm}$ flexion/extension moments while the flexion/extension angle was normalized to the position with $0 \mathrm{Nm}$ flexion/extension. There was no statistically significant difference between the protocols however a trend of decreasing RROM as result of added loading was present. 
Flexibility data are commonly reported in literature as the instantaneous slope of the moment-angle curves of a bending test at key points in the bend. ${ }^{9,20,62}$ Commonness aside, the published methodologies for discerning this data are remarkably cryptic and seemingly subjective flagging this metric as potentially problematic when considering it for protocol comparison. Therefore, author has relegated comments on flexibility to the qualitative realm. The curve presented in Figure 4.7 shows typical flexion/extension flexibility shape with hysteresis. Although variation in curve shape was protocol dependent, no trend was universal enough to merit quantitative investigation.

To the author's knowledge, APT data in the literature are determined by numerous methodologies that render the data incomparable. The lack of a standard APT measurement methodology causes the literature to lack a benchmark. The current study shows several significant differences amidst the APT data. However, when considering the change in APT data, the relative translation that is caused by bending in each protocol is quite similar across all protocols. In other words, the absolute APT data exhibits statistically significant differences at the analysis points between protocols, but the relative translations between the analysis points within each protocol are so similar that when relative translations are statistically evaluated, no significant difference exists across protocols. Therefore the statistical significance observed in translation due to different protocols may be attributed to the initial loading condition only, suggesting that the mechanics of the bending specimen are not significantly altered by either a $400 \mathrm{~N}$ purely compressive load or a $400 \mathrm{~N}$ load of slightly changing direction. However, further investigation may suggest that $400 \mathrm{~N}$ was merely an insufficient magnitude to elicit a significant change in L4-L5 sagittal plane bending mechanics. Further investigation into shear flexibility properties of this joint are merited, particularly with interest in how bending moment affects flexibility. The cause of the apparent flexibility of the joint during bending may be increased pressure in the disc ${ }^{4,12}$ or possibly the engagement of the facet joints and other intact ligaments. ${ }^{32,35,36}$

The current objective in biomechanical testing protocols is to apply "isolated" moments and forces in order to develop repeatable data for quantitative comparison. ${ }^{6}$ The simplicity of isolated application is not derived by neglecting the complexity of in vivo loading circumstances. On the contrary, the simplicity is caused by the difficulties involved in recreating such a complex environment in a manner that is repeatable. This has resulted in a few protocols for measuring spine kinematics that are remarkably telling and useful. However it is still worthwhile to develop new protocols to better simulate the in vivo environment rather than hope that when researchers better understand the environment, perhaps the artifact loads which plague conventional platforms should turn out to be present in vivo, rendering them ingenious instead of unwanted. ${ }^{6}$ Current methods for protocol application are capable of applying new ideas, but not without difficulty. Even the most advanced load-control attempts are still only proven in PM application. $^{24,44,45}$ For this reason, among others, a RTLC robotic testing platform was developed and employed in the intentional application of a force vector along with bending, regardless of the hypothesized difference it could make on spine kinematics. The TW protocol follows the logic that gravity acts upon mass situated above the spine and due to the anatomic angle of L4-L5 in most human beings that vertical force would 


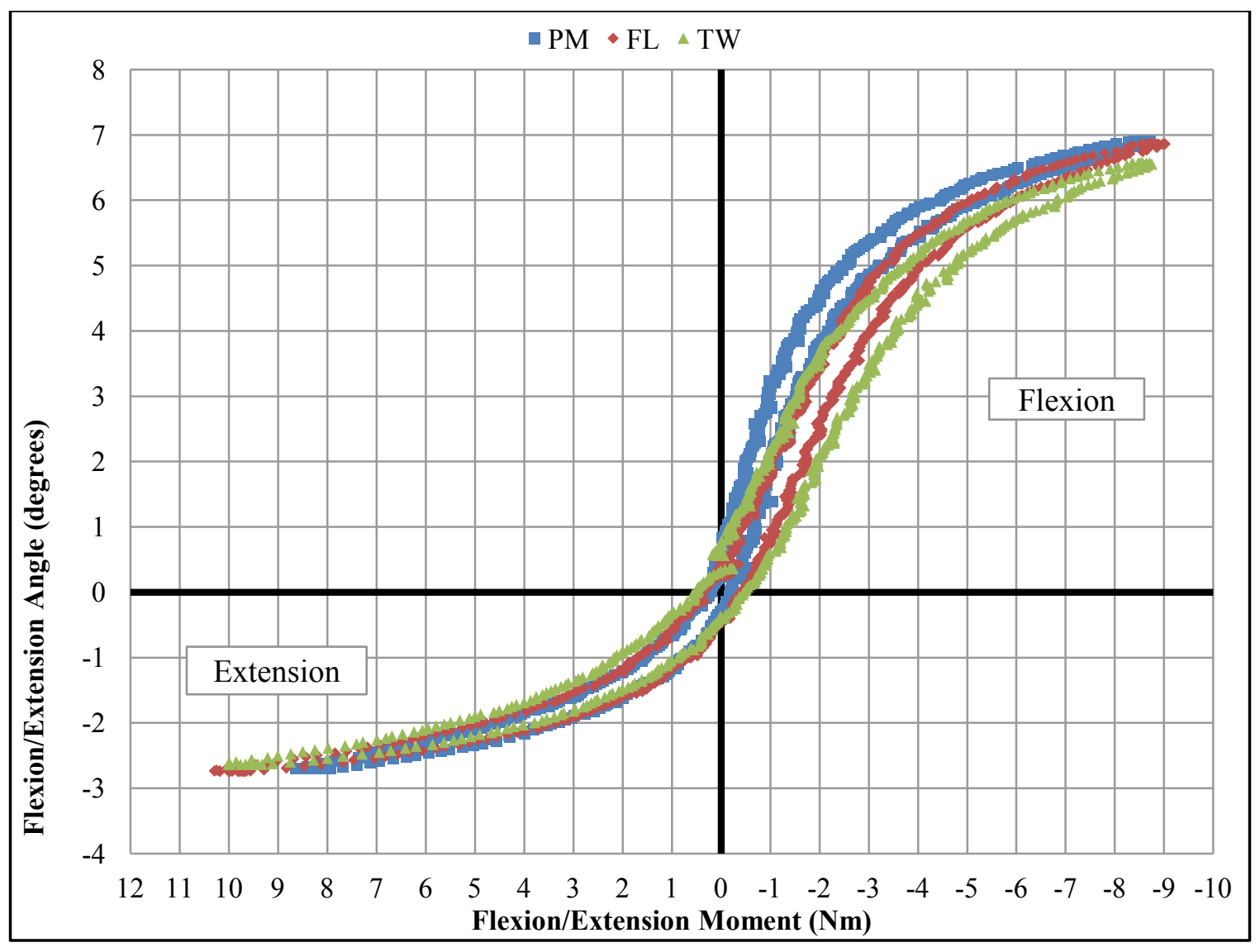

Figure 4.7 Single specimen sagittal bend flexibility comparison across protocols

All three protocols exhibited expected hysteresis characteristics when observing rotational flexibility. There was no drastic difference in most cases but in this example there is a trend of decreased flexibility in the NZ similar to the findings of Patwardhan et al. ${ }^{9}$

Source: A. G. Patwardhan, R. M. Havey, G. Carandang, J. Simonds, L. I. Voronov, A. J. Ghanayem, K. P. Meade, T. M. Gavin and O. Paxinos, 2003. Effect of compressive follower preload on the flexion-extension response of the human lumbar spine. Journal of Orthopaedic Research 21, 540-546. 
result in compression and shear. Although few significant differences between FL and TW were observed this protocol is still useful. Applying another component to the already proven protocols has the potential to change understood in vitro spinal kinematics, decreasing the gap between the bench top and the body. More specifically the TW protocol offers new observations into the biomechanics of the spine as they respond to shear forces.

Conventional FL application as it is applied to full lumbar segments to fulfill its original purpose (to allow the spine to be stable at high compressive loads and not buckle $)^{8,9,12}$ only operates in the sagittal plane. Although this parameter limits only those tests that utilize the original design for FL application (directed cable load) for full lumbar, numerous tests have been conducted involving single segments and non-sagittal mimicking of the FL. This study's comparison involved many changes to conventional practice, therefore the author saw fit to limit application to the sagittal plane, however platform capabilities do not limit the application of load to any one plane. Also the FRF transformation was constant with respect to the mobile FMS throughout bending. This is an assumption against which there is suggestive evidence published in literature. ${ }^{65}$ However the platform has the capability to apply an adaptive dynamic FRF. This capability will likely be investigated as to its implications.

Potential further investigation using this testing platform includes but is not limited to: expanding the planes of bending, increasing the load magnitude, developing standard objective methods for evaluating spine stiffness, NZ, and the inflection point between flexion and extension, and finding the instantaneous center of rotation (ICR) during loading and bending. And although it is hypothesized the load magnitude of $400 \mathrm{~N}$ accounted for the lack of statistical significance, increasing the specimen pool would only better exemplify the population and potentially increase the likelihood of statistically significant differences. 


\section{CHAPTER 5. CONCLUSIONS AND LIMITATIONS}

The first objective of this thesis was to develop a methodology for applying a dynamic force vector during a biomechanical test utilizing the novel robotic RTLC, biomechanical testing platform in the MRL at the UTHSC. As mentioned at the conclusion of Chapter 3, the performance results of the platform when compared to other robotic biomechanical testing platforms was excellent in the realm of TEs and controllability during non-zero targeted commands. Other published robotic testing platforms have only been tested in zero-targeted command protocols and have had limited success with the range of TEs that is considered reasonable. The single MSU (L4L5) biomechanical data observed in this study were comparable to data observed from other non-robotic, FL-based protocols.

The second objective of this thesis was to quantitatively compare three biomechanical tests: PM, ideal FL, and TW protocols. With the aforementioned ability to apply a dynamic force vector during an isolated bending test, the platform robotically conducted the protocols excellently, with acceptable TEs and controllability. The three protocols were compared utilizing the metrics of RROM and APT, the latter of the two being novel in its use as a protocol comparison metric. Statistically significant differences were observed APT but not in RROM. However, the observations of Patwardhan et al. $(2003)^{9}$ regarding a decrease in RROM due to applied compressive load were apparent in the current data. The use of $400 \mathrm{~N}$ as a resultant force magnitude, although deemed reasonable by literature, ${ }^{48,49,50}$ may not have been enough to illicit a statistically significant response.

Some limitations exist in this research but they are not limitations of the CRBT. The point of reactive force within the MSU joint was held at a constant position with respect to the FMS and was specific to specimen. Although literature suggests that the

IPR is mobile under sagittal bending, ${ }^{6,9,40}$ a simplification of procedure was deemed prudent in this initial attempt at protocol development.

The use of single segment MSUs was also a limitation of this study. Much of the motivation for the development of the FL protocol was to find a mechanism for stabilizing the multi-MSU spine such that it would be able to bear in vivo load magnitudes in an in vitro testing environment. ${ }^{12}$ The various limitations of the FL protocol that followed were reasonably accepted so that a full lumbar spine could be tested under more appropriate loads without buckling. The fact that this study used single segments does not in any way devalue the data drawn from this study or denounce the protocol capabilities of the platform. However it should be noted that various difficulties inherent in the application of multi-segment FL were evaded by focusing on a single segment.

As mentioned in Chapter 2, the CRBT possesses six independently controlled DOF, each having the capability of being driven in position- or load-control, and although they are driven independently, they can operate in coordination during motion 
programs. The operation of the platform for this research did not take full advantage of the six DOF load-control capabilities. This is not necessarily seen as a limitation but rather as an operational preference. The constant velocity offered by position-control in the sagittal bending plane allowed for characteristic in vivo movement. Six DOF loadcontrol capabilities set the CRBT apart from most other robotic testing platforms and as such is an attribute worth mentioning, but not at the expense of the value of positioncontrol.

The data from this thesis are also limited to the sagittal plane. Most conventional applications of FL are bound to this plane because of the arrangement of cables and guides. This therefore does not leave the limitation of this study to be unexpected. However this chosen limitation is not as a result of necessity. In applying protocols to single segments, the source of the limitation to one plane is removed and therefore should be noted that the limitation to the sagittal plane is yet another simplification. Expansion into other planes like lateral bending and $\mathrm{CC}$ axial rotation would require more complex algorithms to deal with the effects of coupled bending between those two anatomical planes, ${ }^{2,4}$ making the control of a resultant force vector a far more involved procedure. 


\section{CHAPTER 6. RECOMMENDATIONS FOR FUTURE WORK}

The first recommendation for future work is to conduct a study on the IPR, correlated with the FRF applied in this research, within a single MSU as it changes during bending and torsion. This information would be invaluable when it comes to developing further spine models and IVD replacement implants. This study could be conducted in conjunction with developing more data sets for investigation of the ICR. Conclusions can also be made regarding the relationship between the IPR and the ICR.

Another future work endeavor would be to develop a protocol that simulates FL and TW protocols but also utilizes all six of the CRBT's DOFs of load-control rather than just five as in this research. This may involve implementing the adaptive capabilities of the PID controller to allow the Cartesian manipulator to control forces ably through the $\mathrm{NZ}$ of a spine where the plant flexibility of the tissue changes drastically. Developing systemic checks on motor speeds also may aid in the controllability of the CRBT while progressing through the NZ during bending or torsion.

The most obvious future work endeavor would be to expand the application of protocols to non-sagittal planes. The CRBT's six DOF capabilities suggest promise in fluid application of protocols in lateral bending and $\mathrm{CC}$ axial torsion for PM and protocols incorporating compressive loads normal to the IVD with limited use of position-control axes. But the CRBT also makes possible future investigations into planes not considered anatomically standard. Fully load-controlled coupled bending involving sagittal, lateral, and axial bends is one example of a potential application. The universality of this platform encourages expansion into previously untested realms of biomechanics.

Also, if only for purely academic purposes, material properties of an MSU can be determined with relative ease using this system. Compressive and shear flexibility properties of an MSU with respect to bending would be a worthy data set to possess. The CRBT is a prime candidate for developing in vitro data sets to back computational models of single MSU and potentially full spines.

Finally, expansion into other biomechanical joints presents opportunities for more use and provides broader needs for biomechanical testing. 


\section{LIST OF REFERENCES}

1 M. Nordin and V. H. Frankel, 2001. Basic biomechanics of the musculoskeletal system, 3rd ed. Lippincott Williams \& Wilkins, Philadelphia.

2 R. Cailliet, 2004. The illustrated guide to functional anatomy of the musculoskeletal system. AMA Press, Chicago.

3 R. Chou, J. Baisden, E. J. Carragee, D. K. Resnick, W. O. Shaffer and J. D. Loeser, 2009. Surgery for low back pain: A review of the evidence for an american pain society clinical practice guideline. Spine (Phila Pa 1976) 34, 10941109.

4 A. A. White and M. M. Panjabi, 1990. Clinical biomechanics of the spine, 2nd ed. Lippincott, Philadelphia.

5 A. Rohlmann, G. Bergmann, F. Graichen and U. Weber, 1997. Comparison of loads on internal spinal fixation devices measured in vitro and in vivo. Medical Engineering and Physics 19, 539-546.

$6 \quad$ P. A. Cripton, S. B. Bruehlmann, T. E. Orr, T. R. Oxland and L. P. Nolte, 2000. In vitro axial preload application during spine flexibility testing: Towards reduced apparatus-related artefacts. Journal of Biomechanics 33, 1559-1568.

7 T. Miura, M. M. Panjabi and P. A. Cripton, 2002. A method to simulate in vivo cervical spine kinematics using in vitro compressive preload. Spine (Phila Pa 1976) $27,43-48$.

8 A. G. Patwardhan, R. M. Havey, A. J. Ghanayem, H. Diener, K. P. Meade, B. Dunlap and S. D. Hodges, 2000. Load-carrying capacity of the human cervical spine in compression is increased under a follower load. Spine (Phila Pa 1976) 25, 1548-1554.

9 A. G. Patwardhan, R. M. Havey, G. Carandang, J. Simonds, L. I. Voronov, A. J. Ghanayem, K. P. Meade, T. M. Gavin and O. Paxinos, 2003. Effect of compressive follower preload on the flexion-extension response of the human lumbar spine. Journal of Orthopedic Research 21, 540-546.

10 H. J. Wilke, L. Claes, H. Schmitt and S. Wolf, 1994. A universal spine tester for in vitro experiments with muscle force simulation. European Spine Journal 3, 9197.

11 M. M. Panjabi, T. Miura, P. A. Cripton, J. L. Wang, A. S. Nain and C. Dubois, 2001. Development of a system for in vitro neck muscle force replication in whole cervical spine experiments. Spine (Phila Pa 1976) 26, 2214-2219. 
12 A. G. Patwardhan, R. M. Havey, K. P. Meade, B. Lee and B. Dunlap, 1999. A follower load increases the load-carrying capacity of the lumbar spine in compression. Spine (Phila Pa 1976) 24, 1003-1009.

13 V. K. Goel, S. Goyal, C. Clark, K. Nishiyama and T. Nye, 1985. Kinematics of the whole lumbar spine. Effect of discectomy. Spine (Phila Pa 1976) 10, 543-554.

14 H. J. Wilke, A. Rohlmann, S. Neller, M. Schultheiss, G. Bergmann, F. Graichen and L. E. Claes, 2001. Is it possible to simulate physiologic loading conditions by applying pure moments? A comparison of in vivo and in vitro load components in an internal fixator. Spine (Phila Pa 1976) 26, 636-642.

15 D. J. Diangelo, J. L. Scifert, S. Kitchel, G. B. Cornwall and B. J. Mcvay, 2002. Bioabsorbable anterior lumbar plate fixation in conjunction with cage-assisted anterior interbody fusion. Journal of Neurosurgery 97, 447-455.

16 B. W. Cunningham, J. D. Gordon, A. E. Dmitriev, N. Hu and P. C. Mcafee, 2003. Biomechanical evaluation of total disc replacement arthroplasty: An in vitro human cadaveric model. Spine (Phila Pa 1976) 28, S110-117.

17 J. P. Dickey and K. A. Gillespie, 2003. Representation of passive spinal element contributions to in vitro flexion-extension using a polynomial model: Illustration using the porcine lumbar spine. Journal of Biomechanics 36, 883-888.

18 D. J. Diangelo and K. T. Foley, 2004. An improved biomechanical testing protocol for evaluating spinal arthroplasty and motion preservation devices in a multilevel human cadaveric cervical model. Journal of Neurosurgery: Neurosurgical Focus 17, E4.

19 W. Schmoelz, K. D. Schaser, C. Knop, M. Blauth and A. C. Disch, 2010. Extent of corpectomy determines primary stability following isolated anterior reconstruction in a thoracolumbar fracture model. Clinical Biomechanics (Bristol, Avon) 25, 16-20.

20 D. J. Wheeler, A. L. Freeman, A. M. Ellingson, D. J. Nuckley, J. M. Buckley, J. K. Scheer, N. R. Crawford and J. E. Bechtold, 2011. Inter-laboratory variability in in vitro spinal segment flexibility testing. Journal of Biomechanics 44, 23832387.

21 M. M. Panjabi, M. H. Krag, A. A. White, 3rd and W. O. Southwick, 1977. Effects of preload on load displacement curves of the lumbar spine. Orthopedic Clinincs of North America 8, 181-192.

22 J. Hamill and K. Knutzen, 2003. Biomechanical basis of human movement, 2nd ed. Lippincott Williams \& Wilkins, Philadelphia.

23 D. M. Gorinevski1̌, A. M. Formal'Skil̆ and A. I. U. ShneiّDer, 1997. Force control of robotics systems. CRC press, Boca Raton. 
24 L. G. Gilbertson, T. C. Doehring and J. D. Kang, 2000. New methods to study lumbar spine biomechanics: Delineation of in vitro load-displacement characteristics by using a robotic/ufs testing system with hybrid control. Operative Techniques in Orthopaedics 10, 246-253.

25 H. Fujie, T. Sekito and A. Orita, 2004. A novel robotic system for joint biomechanical tests: Application to the human knee joint. Journal of Biomechanical Engineering 126, 54.

26 J. Butler, J. W. Hole, R. Lewis and D. Shier, 2002. Hole's human anatomy \& physiology, 9th ed. McGraw-Hill, Boston.

27 D. A. Winter, 1979. Biomechanics of human movement. Wiley, New York.

28 E. C. Benzel, 2005. Spine surgery : Techniques, complication avoidance, and management, 2nd ed. Churchill Livingstone, Philadelphia, Pa.

29 M. D. Humzah and R. W. Soames, 1988. Human intervertebral disc: Structure and function. The Anatomical Record 220, 337-356.

30 C. Q. Zhao, L. M. Wang, L. S. Jiang and L. Y. Dai, 2007. The cell biology of intervertebral disc aging and degeneration. Ageing Research Reviews 6, 247-261.

31 K. D. Fraysur, 2010. A passive pure moment protocol for testing spine segments: Development and application. University of Tennessee Health Science Center and University of Memphis, Memphis, TN.

32 M. M. Panjabi, 1992. The stabilizing system of the spine. Part ii. Neutral zone and instability hypothesis. Journal of Spinal Disorders 5, 390-396; discussion 397.

33 M. M. Panjabi, K. Abumi, J. Duranceau and J. J. Crisco, 1988. Biomechanical evaluation of spinal fixation devices: Ii. Stability provided by eight internal fixation devices. Spine (Phila Pa 1976) 13, 1135-1140.

34 T. Yoshioka, H. Tsuji, N. Hirano and S. Sainoh, 1990. Motion characteristic of the normal lumbar spine in young adults: Instantaneous axis of rotation and vertebral center motion analyses. Journal of Spinal Disorders 3, 103-113.

35 M. M. Panjabi, 1992. The stabilizing system of the spine. Part i. Function, dysfunction, adaptation, and enhancement. Journal of Spinal Disorders 5, 383389; discussion 397.

36 M. M. Panjabi, T. R. Oxland, I. Yamamoto and J. J. Crisco, 1994. Mechanical behavior of the human lumbar and lumbosacral spine as shown by threedimensional load-displacement curves. Journal of Bone and Joint Surgery 76, 413-424. 
37 D. J. Diangelo, J. T. Roberston, N. H. Metcalf, B. J. Mcvay and R. C. Davis, 2003. Biomechanical testing of an artificial cervical joint and an anterior cervical plate. Journal of Spinal Disorders and Techniques 16, 314-323.

38 J. P. Dickey and D. J. Kerr, 2003. Effect of specimen length: Are the mechanics of individual motion segments comparable in functional spinal units and multisegment specimens? Medical Engineering and Physics 25, 221-227.

39 C. A. Reitman, K. M. Mauro, L. Nguyen, J. M. Ziegler and J. A. Hipp, 2004. Intervertebral motion between flexion and extension in asymptomatic individuals. Spine (Phila Pa 1976) 29, 2832-2843.

40 W. H. Chuang, S. C. Lin, S. H. Chen, C. W. Wang, W. C. Tsai, Y. J. Chen and J. R. Hwang, 2012. Biomechanical effects of disc degeneration and hybrid fixation on the transition and adjacent lumbar segments: Trade-off between junctional problem, motion preservation, and load protection. Spine (Phila Pa 1976) 37, E1488-1497.

41 V. K. Goel, D. G. Wilder, M. H. Pope and W. T. Edwards, 1995. Biomechanical testing of the spine. Load-controlled versus displacement-controlled analysis. Spine (Phila Pa 1976) 20, 2354-2357.

42 J. T. Lysack, J. P. Dickey, G. A. Dumas and D. Yen, 2000. A continuous pure moment loading apparatus for biomechanical testing of multi-segment spine specimens. Journal of Biomechanics 33, 765-770.

43 J. Eguizabal, M. Tufaga, J. K. Scheer, C. Ames, J. C. Lotz and J. M. Buckley, 2010. Pure moment testing for spinal biomechanics applications: Fixed versus sliding ring cable-driven test designs. Journal of Biomechanics 43, 1422-1425.

44 D. J. Goertzen and G. N. Kawchuk, 2009. A novel application of velocity-based force control for use in robotic biomechanical testing. Journal of Biomechanics 42, 366-369.

45 M. R. Walker and J. P. Dickey, 2007. New methodology for multi-dimensional spinal joint testing with a parallel robot. Medical and Biological Engineering and Computing 45, 297-304.

46 D. M. Wido, 2011. Use of a spine robot employing a real time force control algorithm to develop, simulate, and compare spinal biomechanical testing protocols: Eccentric loading, pure moment, and a novel head weight protocol. University of Tennessee Health Science Center and University of Memphis, Memphis, TN.

47 B. P. Kelly and C. R. Bennett, 2013. Design and validation of a novel cartesian biomechanical testing system with coordinated 6dof real-time load control: Application to the lumbar spine (11-s, 14-15). Journal of Biomechanics. 

Ghanayem, R. M. Havey, G. Carandang, M. Sartori, I. N. Gaitanis, S. Fronczak and A. G. Patwardhan, 2005. Response of charite total disc replacement under physiologic loads: Prosthesis component motion patterns. Spine Journal 5, 590599.

49 J. L. Gaffey, A. J. Ghanayem, M. L. Voronov, R. M. Havey, G. Carandang, C. Abjornson and A. G. Patwardhan, 2010. Effect of increasing implant height on lumbar spine kinematics and foraminal size using the prodisc-1 prosthesis. Spine (Phila Pa 1976) 35, 1777-1782.

50 S. M. Renner, P. P. Tsitsopoulos, A. T. Dimitriadis, L. I. Voronov, R. M. Havey, G. Carandang, B. Mcintosh, C. Carson, D. Ty, J. G. Ringelstein and A. G. Patwardhan, 2011. Restoration of spinal alignment and disk mechanics following polyetheretherketone wafer kyphoplasty with staxx fx. American Journal of Neuroradiology 32, 1295-1300.

51 I. Yamamoto, M. M. Panjabi, T. Crisco and T. Oxland, 1989. Three-dimensional movements of the whole lumbar spine and lumbosacral joint. Spine (Phila Pa 1976) $14,1256-1260$.

52 D. J. Goertzen, C. Lane and T. R. Oxland, 2004. Neutral zone and range of motion in the spine are greater with stepwise loading than with a continuous loading protocol. An in vitro porcine investigation. Journal of Biomechanics 37, 257-261.

53 H. J. Wilke, K. Wenger and L. Claes, 1998. Testing criteria for spinal implants: Recommendations for the standardization of in vitro stability testing of spinal implants. European Spine Journal 7, 148-154.

54 V. K. Goel, M. M. Panjabi, A. G. Patwardhan, A. P. Dooris, H. Serhan, T. American Society For and Materials, 2006. Test protocols for evaluation of spinal implants. Journal of Bone and Joint Surgery 88 Suppl 2, 103-109.

55 M. M. Panjabi, 2007. Hybrid multidirectional test method to evaluate spinal adjacent-level effects. Clinical Biomechanics (Bristol, Avon) 22, 257-265.

56 H. Bozkus, M. Senoglu, S. Baek, A. G. Sawa, A. F. Ozer, V. K. Sonntag and N. R. Crawford, 2010. Dynamic lumbar pedicle screw-rod stabilization: In vitro biomechanical comparison with standard rigid pedicle screw-rod stabilization. Journal of Neurosurgery: Spine 12, 183-189.

57 C. K. Demetropoulos, D. K. Sengupta, M. A. Knaub, B. P. Wiater, C. Abjornson, E. Truumees and H. N. Herkowitz, 2010. Biomechanical evaluation of the kinematics of the cadaver lumbar spine following disc replacement with the prodisc-1 prosthesis. Spine (Phila Pa 1976) 35, 26-31. 
58 M. Schulze, R. Hartensuer, D. Gehweiler, U. Holscher, M. J. Raschke and T. Vordemvenne, 2012. Evaluation of a robot-assisted testing system for multisegmental spine specimens. Journal of Biomechanics doi:10.1016/j.jbiomech.2012.02.013.

59 J. J. Crisco, 3rd, X. Chen, M. M. Panjabi and S. W. Wolfe, 1994. Optimal marker placement for calculating the instantaneous center of rotation. Journal of Biomechanics 27, 1183-1187.

60 V. Goel, A. Mehta, J. Jangra, A. Faizan, A. Kiapour, R. Hoy and R. Fauth, 2007. Anatomic facet replacement system (afrs) restoration of lumbar segment mechanics to intact: A finite element study and in vitro cadaver investigation. Spine Arthroplasty Society Journal 01, 46-54.

61 J. A. Miller and L. B. Skogland, 1980. On the load-displacement behaviour of adolescent spinal motion segments - an experimental study using autopsy specimens. University of Oslo, Oslo, Norway.

62 J. Janevic, J. A. Ashton-Miller and A. B. Schultz, 1991. Large compressive preloads decrease lumbar motion segment flexibility. Journal of Orthopedic Research 9, 228-236.

63 A. Rohlmann, S. Neller, L. Claes, G. Bergmann and H. J. Wilke, 2001. Influence of a follower load on intradiscal pressure and intersegmental rotation of the lumbar spine. Spine (Phila Pa 1976) 26, E557-561.

64 C. A. Niosi, Q. A. Zhu, D. C. Wilson, O. Keynan, D. R. Wilson and T. R. Oxland, 2006. Biomechanical characterization of the three-dimensional kinematic behaviour of the dynesys dynamic stabilization system: An in vitro study. European Spine Journal 15, 913-922.

65 D. G. Wilder, M. H. Pope, R. E. Seroussi, J. Dimnet and M. H. Krag, 1989. The balance point of the intervertebral motion segment: An experimental study. Bulletin of the Hospital for Joint Diseases Orthopaedic Institute 49, 155-169. 
APPENDIX A. HARDWARE COMPONENTS AND SPECIFICATIONS

Table A.1 Individual Linear Axis Hardware Specifications

\begin{tabular}{lccc}
\hline Feature & X-axis & Y-axis & Z-axis \\
\hline Make/Model & Parker MPP0922 & Parker MPP0922 & Exlar IX30 Linear \\
& Servo Motor \& & Servo Motor \& & Roller Screw \\
& $5 \mathrm{~mm}$ Ball Screw & $2 \mathrm{~mm}$ Ball Screw & Actuator \\
$\mathrm{ROM}_{\max }$ & $673 \mathrm{~mm}$ & $457 \mathrm{~mm}$ & $279 \mathrm{~mm}$ \\
Encoder Resolution & $<1.0 \mu \mathrm{m}$ & $<1.0 \mu \mathrm{m}$ & $<1.0 \mu \mathrm{m}$ \\
Accuracy & $\pm 0.3 \mu \mathrm{m}$ & $0.2 \mu \mathrm{m}$ & $0.2 \mu \mathrm{m}$ \\
Continuous Load & $2.5 \mathrm{kN}$ & $6.5 \mathrm{kN}$ & $5.0 \mathrm{kN}$ \\
\hline
\end{tabular}

The $\mathrm{x}$ - and $\mathrm{y}$-axes are comprised of a commercially available servo motor and ball screw assemblies combined in-house with custom designed and machined parts. Encoder resolution, accuracy, and continuous load ratings are from manufacturer specifications.

Table A.2 Individual Rotary Axis Hardware Specifications

\begin{tabular}{lccc}
\hline Feature & Roll & Pitch & Yaw \\
\hline Make/Model & Harmonic Drive & Harmonic Drive & Harmonic Drive \\
& FHA-25C-100- & FHA-25C-100- & FHA-17C-50- \\
& US250-F5 & US250-F5 & US250-F5 \\
ROM $_{\max }$ & $\pm 180^{\circ}$ & $\pm 180^{\circ}$ & $\pm 180^{\circ}$ \\
Encoder Resolution & 13 arc-sec & 13 arc-sec & 26 arc-sec \\
Accuracy & 60 arc-sec & 60 arc-sec & 60 arc-sec \\
Continuous Load & $120 \mathrm{Nm}$ & $120 \mathrm{Nm}$ & $23 \mathrm{Nm}$ \\
\hline
\end{tabular}

Encoder resolution, accuracy, and continuous load ratings are from manufacturer specifications.

Table A.3 JR3 Model 45E15A4-I63-AF Force Moment Sensor Specifications

\begin{tabular}{lccc}
\hline Feature & $\mathrm{F}_{\mathrm{x}}, \mathrm{F}_{\mathrm{y}}$ & $\mathrm{F}_{\mathrm{z}}$ & $\mathrm{M}_{\mathrm{x}}, \mathrm{M}_{\mathrm{y}}, \mathrm{M}_{\mathrm{z}}$ \\
\hline Capacity & $444 \mathrm{~N}$ & $888 \mathrm{~N}$ & $50.8 \mathrm{Nm}$ \\
Resolution & $0.06 \mathrm{~N}$ & $0.11 \mathrm{~N}$ & $0.006 \mathrm{Nm}$ \\
Accuracy & $\pm 1.1 \mathrm{~N}$ & $\pm 2.2 \mathrm{~N}$ & $\pm 0.1 \mathrm{Nm}$ \\
\hline
\end{tabular}




\section{APPENDIX B. FORCE/TORQUE CONTROL ALGORITHM}

The following text comprise the two compiled programmable logic commands (PLCC) that constitute the user definitions governing the variable characteristics of the cascaded control loop within the Delta Tau controller. Several variables will be without detailed definition however this text along with the UMAC user's manual and Delta Tau Software Reference Manual should shed some light into the inner workings of the control system.

Lines of code within the controller will be shown in the Courier New font. Any line of code that begins with a semicolon should be regarded as documentation meant for user clarification within the code.

\section{A.1 PLCC 0}

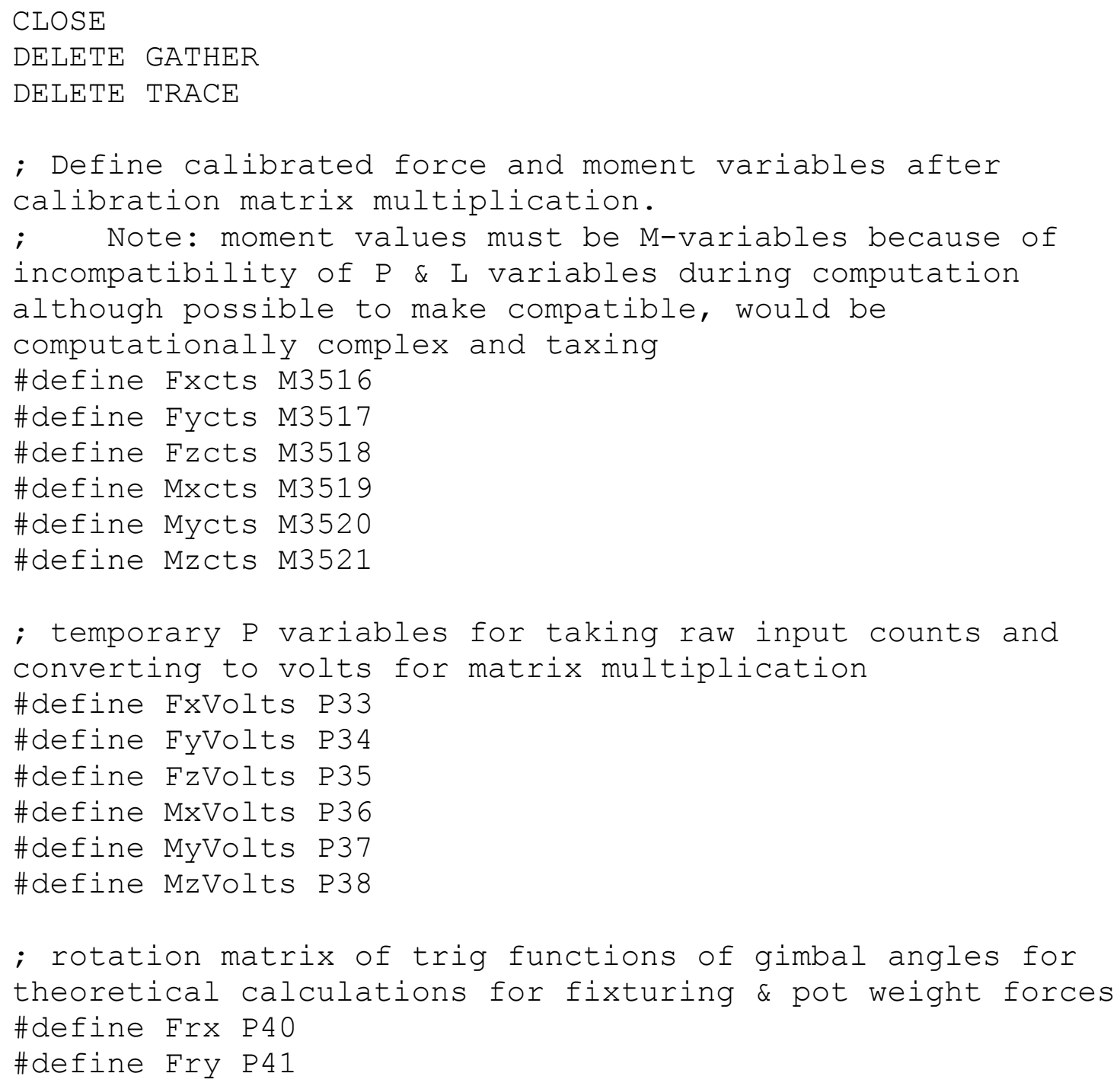


\#define Frz P42

; theoretical load cell readings for moments caused by gravity on fixturing including pot weight

\#define Max P43

\#define May P44

\#define Maz P45

; measured values for force reference frame offset

displacement with respect to the center of the load cell

(units meters)

\#define frfdx P46

\#define frfdy P47

\#define frfdz P48

; measured and empirical values for center of mass of

fixturing and pot material displacement from load cell

center

; \#define dxf P50 ; commented out b/c it equals zero

; \#define dyf P51 ; commented out b/c it equals zero

\#define dzf P52

\#define dxp P53

\#define dyp P54

\#define dzp P55

; measured and empirical values for weight of fixturing and pots

\#define FIX P56

\#define POT P57

; M4000->X:\$351C, 24, S

; M3531->Y:\$78202, 24, S

$; \mathrm{M} 3531=\mathrm{M} 4000$

; L variable pointers to output registers of A/D board for $\mathrm{LC} 1$

iformerly used output registers for ECT motors \#9 - \#14

L9->Y:\$78C00,8,16 ;formerly M3509 (X:\$3509,24,S)

L10->Y:\$78C01,8,16 ;formerly M3510 (X:\$350A, 24,S)

L11->Y:\$78C02,8,16 ; formerly M3511 (X:\$350B, 24,S)

L12->Y:\$78C03,8,16 ;formerly M3512 (X:\$350C,24,S)

L13->Y:\$78D00,8,16 ; formerly M3513 (X:\$350D, 24,S)

L14->Y:\$78D01,8,16 ;formerly M3514 (X:\$350E, 24,S)

; Pointers assigned to free global registers to hold output (calibrated force values) from open servo 
; These global free registers are set up in the ECT as source registers for parallel byte wide conversion of data ; The values in these registers are to be passed to motor position registers for motors \#16 to \#21 for force feedback.

M3516->Y:\$10F0,0,24, S M3517->Y:\$10F1, 0,24, S M3518->Y: $\$ 10 F 2,0,24, S$ M3519->Y:\$10F3,0,24, S M3520->Y: $\$ 10 F 4,0,24, \mathrm{~S}$ M3521->Y: $\$ 10 F 5,0,24, \mathrm{~S}$

; F Variable pointers to angles from gimbal

F31->D:\$20B ;formerly M462

F32->D:\$28B ;formerly M562

F33->D:\$30B ;formerly M662

Open PLCC 0

clear

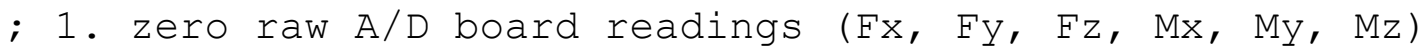
and convert to volts (A/D input is bipolar so 32767 cts = zero force).

; 2. conversion to volts necessary for $6 \times 6$ calibration matrix multiplication.

; (Note: input has been shifted by 5 bits by multiplying by $32: 1048675=32 * 32768)$

; (Note: $(32 * \mathrm{~L} 9-1048576) / 104857=(\mathrm{L} 9-32768) / 3276.78$

\begin{tabular}{|c|c|c|}
\hline $\begin{array}{l}\text { FxVolts }=(\text { ITOF (L9) }-32768) / 3276.78 \\
1048576) / 104857\end{array}$ & ; formerly & $(32 * \mathrm{~L} 9-$ \\
\hline $\begin{array}{l}\text { FyVolts }=(\text { ITOF }(\text { L10 })-32768) / 3276.78 \\
1048576) / 104857\end{array}$ & ; formerly & $(32 * \mathrm{~L} 10-$ \\
\hline $\begin{array}{l}\text { zVolts }=(\text { ITOF }(\text { L11 })-32768) / 3276.78 \\
048576) / 104857\end{array}$ & ; formerly & $(32 * \operatorname{L11-}$ \\
\hline $\begin{array}{l}\text { xVolts }=(\text { ITOF }(\text { L12) }-32768) / 3276.78 \\
048576) / 104857\end{array}$ & ; formerly & $(32 * \mathrm{~L} 12-$ \\
\hline $\begin{array}{l}\text { MyVolts }=(\operatorname{ITOF}(\operatorname{L13})-32768) / 3276.78 \\
1048576) / 104857\end{array}$ & ; formerly & $(32 * \mathrm{~L} 13-$ \\
\hline $\begin{array}{l}\text { zVolts }=(\text { ITOF }(\text { L14 })-32768) / 3276.78 \\
048576) / 104857\end{array}$ & ; formerly & $(32 * \mathrm{~L} 14-$ \\
\hline
\end{tabular}

; rotation matrix of trig functions of gimbal angles for theoretical calculations for fixturing \& pot weight forces $\operatorname{Frx}=(\operatorname{SIN}(\mathrm{F} 31 / 8533331) * \operatorname{SIN}(\mathrm{F} 33 / 4266664)-$ $\operatorname{COS}(F 31 / 8533331) * \operatorname{SIN}(F 32 / 8533331) * \operatorname{COS}(F 33 / 4266664))$ 
Fry $=(\operatorname{COS}(\mathrm{F} 31 / 8533331) * \operatorname{SIN}(\mathrm{F} 32 / 8533331) * \operatorname{SIN}(\mathrm{F} 33 / 4266664)+\mathrm{SIN}$ $(\mathrm{F} 31 / 8533331) * \operatorname{COS}(\mathrm{F} 33 / 4266664))$

$\operatorname{Frz}=(\operatorname{COS}(\mathrm{F} 31 / 8533331) * \operatorname{COS}(\mathrm{F} 32 / 8533331))$

; Theoretical calculations for fixturing \& pot weight forces based on gimbal angles and center of mass displacements in newton meters Max $=-d z f * F I X * F r y-d z p * P O T * F r y$ May $=d z f * F I X * F r x+d z p * P O T * F r x$ Ma $z=d x p * P O T * F r y-d y p * P O T * F r x$

;1. Each line below has matrix multiplication to yield force output in lbs or inch pounds

;2. A conversion to Newtons ( $x$ 4.448) or Newton meters ( $x$ $0,112984)$ is done at the end of each line

;3. A factor is multiplied last to convert back to counts. I believe this factor is arbitrary and can be used as ; the motor axis scaling factor for newtons to counts. For force I used approximately 17 lbs per volt input which

; converts to 1387 counts/newton including multiplying by 32 to maintain that 5 bit shift. Note the ECT entries for parallel

; conversion and feedback for motors 16 to 21 do not have bit shifts. I think this is because it has already occurred

; in the first ECT conversions from the A/D board to motors 9 to 14 .

Fxcts $=(-75.282 *$ FxVolts $-($ FIX + POT $) *$ Frx $) * 1387$

Fycts $=(-75.763 *$ FyVolts $-($ FIX + POT $) *$ Fry $) * 1387$

$\mathrm{Fzcts}=(152.967 * \mathrm{FzVOlts}+(\mathrm{FIX}+\mathrm{POT}) * \mathrm{Frz}) * 693.5$

Mxcts $=(-8.521 *$ MxVolts -

$\operatorname{Max}+($ frfdy* $(M 1862 / 66575.8)+f r f d z *(M 1762 / 133151.5))) * 12292$ ; checked correct 2012.09.27

Mycts $=(-8.562 *$ MyVolts - May-

(frfdz* (M1662/133151.5) +frfdx* (M1862/66575.8))) *12292 ; checked correct 2012.09.27

Mzcts $=(-8.575 *$ MzVolts $-M a z-($ frfdx* (M1762/133151.5) frfdy* (M1662/133151.5)))*12292 ; checked correct 2012.09 .27

CLOSE 


\section{A.2 PLCC 2}

; measured and empirical values for weight of fixturing and pots

\#define BOT P58

\#define BFIX P59

; temporary $\mathrm{P}$ variables for taking raw bottom load cell input and converting to volts for matrix multiplication ; (Note: 5 bit shift applied to simulate transition through $\mathrm{ECT}$ )

\#define FxVolts2 P60

\#define FyVolts2 P61

\#define FzVolts2 P62

\#define MxVolts2 P63

\#define MyVolts2 P64

\#define MzVolts2 P65

; bottom load cell output values after calibration matrix manipulation units are in Newtons and Newton-meters

; (Note: there is a zeroing factor at the end of the calibration matrix that nulls out permanent fixturing)

; formerly P66-P71, THEN M3550-M3555

\#define Fx2 M3550

\#define Fy2 M3551

\#define Fz2 M3552

\#define Mx2 M3553

\#define My2 M3554

\#define Mz2 M3555

; L variable pointers to raw analogue signal from second load cell (250 1b)

L22->Y:\$78D02,8,16 ; formerly M4022

L23->Y:\$78D03,8,16 ; formerly M4023

L24->Y:\$78E00,8,16;formerly M4024

L25->Y:\$78E01,8,16; formerly M4025

L26->Y:\$78E02,8,16 ; formerly M4026

L27->Y:\$78E03,8,16; formerly M4027

; theoretical load cell readings for moments caused by gravity on fixturing including pot weight on load cell 2 \#define Max2 P100 \#define May2 P101

; Maz2 always equals zero because the second load cell never moves 
; measured values for force reference frame offset displacement with respect to the center of the load cell (units meters)

\#define frfdx2 P103

\#define frfdy2 P104

\#define frfdz2 P105

; measured and empirical values for center of mass of fixturing and pot material displacement from load cell center (units meters)

\#define dxf2 P106

\#define dyf2 P107

; \#define dzf2 P108 ; commented out b/c it does not

affect moment adjustment (units meters)

\#define dxp2 P109

\#define dyp2 P110

; \#define dzp2 P111 ; commented out b/c it does not affect moment adjustment (units meters)

; Pointers assigned to free global registers to hold output (calibrated force values) from open servo

; These global free registers are set up in the ECT as source registers for parallel byte wide conversion of data ; The values in these registers are to be passed to motor position registers for motors \#16 to \#21 for force feedback. M3550->X:\$107FA, 0,24, S M3551->X: $\$ 107 \mathrm{FB}, 0,24, \mathrm{~S}$ M3552->X: $\$ 107 \mathrm{FC}, 0,24, \mathrm{~S}$ M3553->X:\$107ED, 0,24, S M3554->X:\$107FE, $0,24, \mathrm{~S}$ M3555->X:\$107FF,0,24, S

OPEN PLCC 2 CLEAR

; 1. zero raw readings from load cell 2 (250 1b) and convert to volts (A/D input is bipolar so 32767 cts $=$ zero force). ; 2. conversion to volts necessary for $6 \times 6$ calibration matrix multiplication.

; (Note: input must be shifted by 5 bits to simulate ECT therefore multiplied by 32:1048675=32*32768)

FxVolts2=(ITOF (L22)-32768)/3276.78; formerly (32*L22$1048576) / 104857$

FYVolts2=(ITOF (L23)-32768)/3276.78; formerly (32*L23$1048576) / 104857$

FzVolts2 $=(\operatorname{ITOF}($ L24)-32768)/3276.78; formerly (32*L24$1048576) / 104857$ 


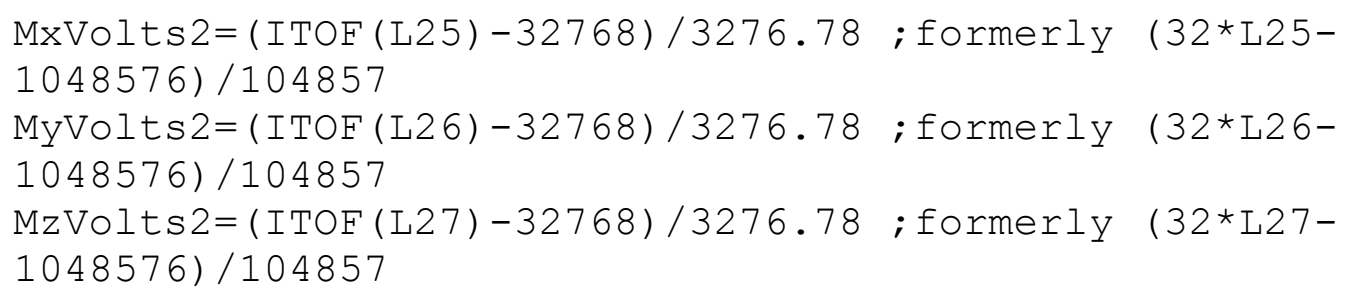

; Theoretical calculations for fixturing \& pot weight forces based on gimbal angles and center of mass displacements in newton meters

$\operatorname{Max} 2=-1 *($ dyf $2 * B F I X+$ dyp $2 * B O T)$

May $2=d x f 2 * B F I X+d x p 2 * B O T$

; 1. Each line below has matrix multiplication to yield force output in lbs or inch pounds

; 2. A conversion to Newtons ( $x$ 4.448) or Newton meters (x $0,112984)$ is done at the end of each line

; (Note: If one of these outputs is to be used as feedback for a motor, it needs to be converted back into counts, which requires

; multiplication by 32 (5 bit shift) multiplied by the motor axis scaling factor for newtons to counts, whatever that may be.

; Calibration matrix values for LC2 have been altered, forces were originally multiplied by 4.448 to convert from lbs to $\mathrm{N}$.

; and moments multiplied by 0.112984 to convert from ft-lb to $\mathrm{Nm}$. those conversion factors have been folded into the ; calibration matrix values to decrease the computational load

$\mathrm{Fx} 2=(-((171.826 * \mathrm{FxVolts} 2)+(-0.249 * \mathrm{FYVolts} 2)+(-$

$1.592 * \mathrm{FzVOlts} 2)+(-1.437 * \mathrm{MxVolts} 2)+(-$

$3.936 *$ MyVolts 2$)+(1.841 *$ MzVolts 2$))-(-23.064)) * 616$

$\mathrm{Fy} 2=(-((-$

$0.080 *$ FxVolts 2$)+(170.478 *$ FyVolts 2$)+(1.672 *$ FzVolts 2$)+(3.852 *$

MxVolts 2$)+(-1.254 *$ MyVolts 2$)+(0.382 *$ MzVolts2) $)-(14.233)) * 616$

$\mathrm{Fz} 2=(((8.078 * \mathrm{FxVolts} 2)+(2.624 * \mathrm{FyVolts} 2)+(378.311 * \mathrm{FzVolts} 2)+$

$(3.327 *$ MxVolts 2$)+(-5.578 *$ MyVolts2 $)+(-11.796 *$ MzVolts 2$))-(-$ $24.465)-\mathrm{BFIX}-\mathrm{BOT}) * 313$

$\mathrm{Mx} 2=(-$

$((0.047 *$ FxVolts 2$)+(0.017 *$ FyVolts 2$)+(0.022 *$ FzVolts 2$)+(20.777$

$\star M x V o l t s 2)+(-0.013 *$ MyVolts2 $)+(-0.602 * M z V o l t s 2))-(-1.986)-$

$\operatorname{Max} 2+($ frfdy2* $(\mathrm{Fz} 2)-\mathrm{frfdz} 2 *(\mathrm{Fy} 2))) * 5044$

My2 $=(-((-0.205 *$ FxVolts 2$)+(0.169 *$ FyVolts 2$)+(-$

$0.136 *$ FzVolts 2$)+(-0.011 * M x V o l t s 2)+(20.823 *$ MyVolts 2$)+(-$ 
$0.307 * \mathrm{MzVolts} 2))-(-0.077)-\mathrm{May} 2+(\mathrm{frfdz} 2 *(\mathrm{Fx} 2)-$ frfdx2*(Fz2)) )*5044

$\mathrm{Mz} 2=(-((0.041 * \mathrm{FxVolts} 2)+(-0.019 * \mathrm{FYVolts} 2)+(-$

$0.037 * \mathrm{FzVOlts} 2)+(0.071 * \mathrm{MxV}$ olts 2$)+(-$

$0.150 *$ MyVolts 2$)+(20.806 * M z V o l t s 2))-(-0.817)+\left(\operatorname{frfdx} 2 *\left(F_{y} 2\right)-\right.$ frfdy2*(Fx2)) )*5044

CLOSE 


\section{APPENDIX C. CUSTOM MOTION PROGRAMS FOR PROTOCOLS}

The following text comprises examples of the three motion programs, Program 14,15 and 16, that were custom written for protocol execution. Several variables will be without detailed definition however this text along with the UMAC user's manual and Delta Tau Software Reference Manual should shed some light onto the testins.

Lines of code within the controller will be shown in the Courier New font. Any line of code that begins with a semicolon or double backslash ('//') should be regarded as documentation meant for user clarification within the code.

\section{B.1 Program 14 Pure Moment Pitch Position}

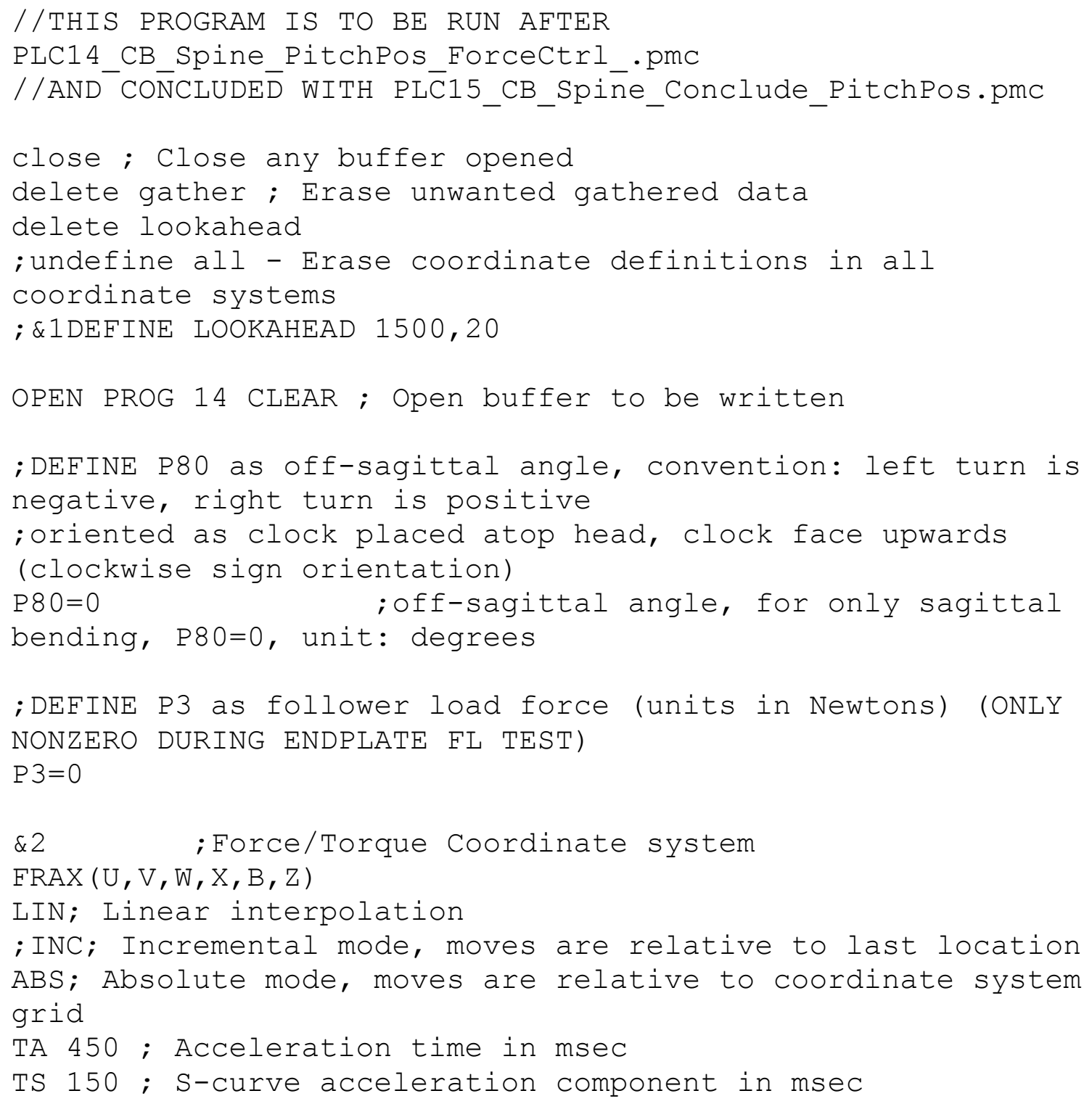


$\& 2 \% 100 \quad ;$ Feedrate Override value

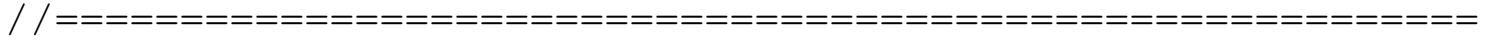
$==\mathrm{=}=\mathrm{=}=\mathrm{=}=\mathrm{=}=\mathrm{=}=\mathrm{=}=\mathrm{=}=\mathrm{=}=\mathrm{=}=$

; this segment puts the MSU into the no load starting point utilizing P82

;P82 is reset before it is utilized for the test in later whileloops

$\operatorname{FRAX}(\mathrm{B})$

TM 1000

$\mathrm{P} 82=0$

IF (M2062>590000)

WHILE (M2062>0) ; MY $\sim 0.5 \mathrm{Nm}$

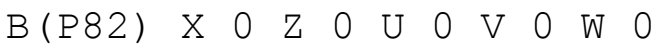

$\mathrm{P} 82=\mathrm{P} 82+.2$

ENDWHILE

$\mathrm{P} 82=\mathrm{P} 82-.2$

ELSE

IF $(\mathrm{M} 2062<-590000)$

WHILE $($ M2062<0) ; My -0.5 Nm

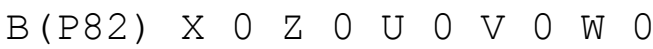

$\mathrm{P} 82=\mathrm{P} 82-.2$

ENDWHILE

$\mathrm{P} 82=\mathrm{P} 82+.2$

ELSE

$P 82=(\operatorname{M5} 62 /(2777.777 * 32 * 96))$

$\begin{array}{llllllllll}\mathrm{U} & 0 & \mathrm{~V} & 0 & \mathrm{~W} & 0\end{array}$

$\mathrm{F} .4$

$\begin{array}{lllll}X & 0 & Z & 0\end{array}$

DWELLO

ENDIF

ENDIF

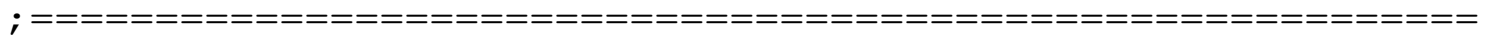

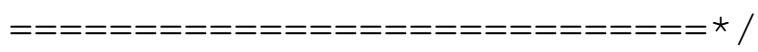

DWELLO

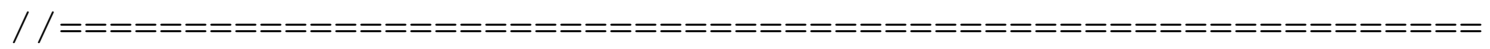

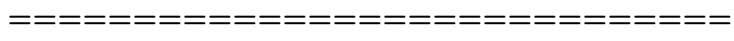

; this segment conducts the force reference frame transform for single segment MSUs

; under the conditions that the program is operating under position control in some capacity

; (not full torque) AND that there is a desired applied

load. Be sure to check the commanded position

; control motor to ensure that the correct motor is being addressed (ex. B is the pitch motor)

IF $\quad(\mathrm{P} 3 !=0)$

P $49=\mathrm{M} 2062$ 


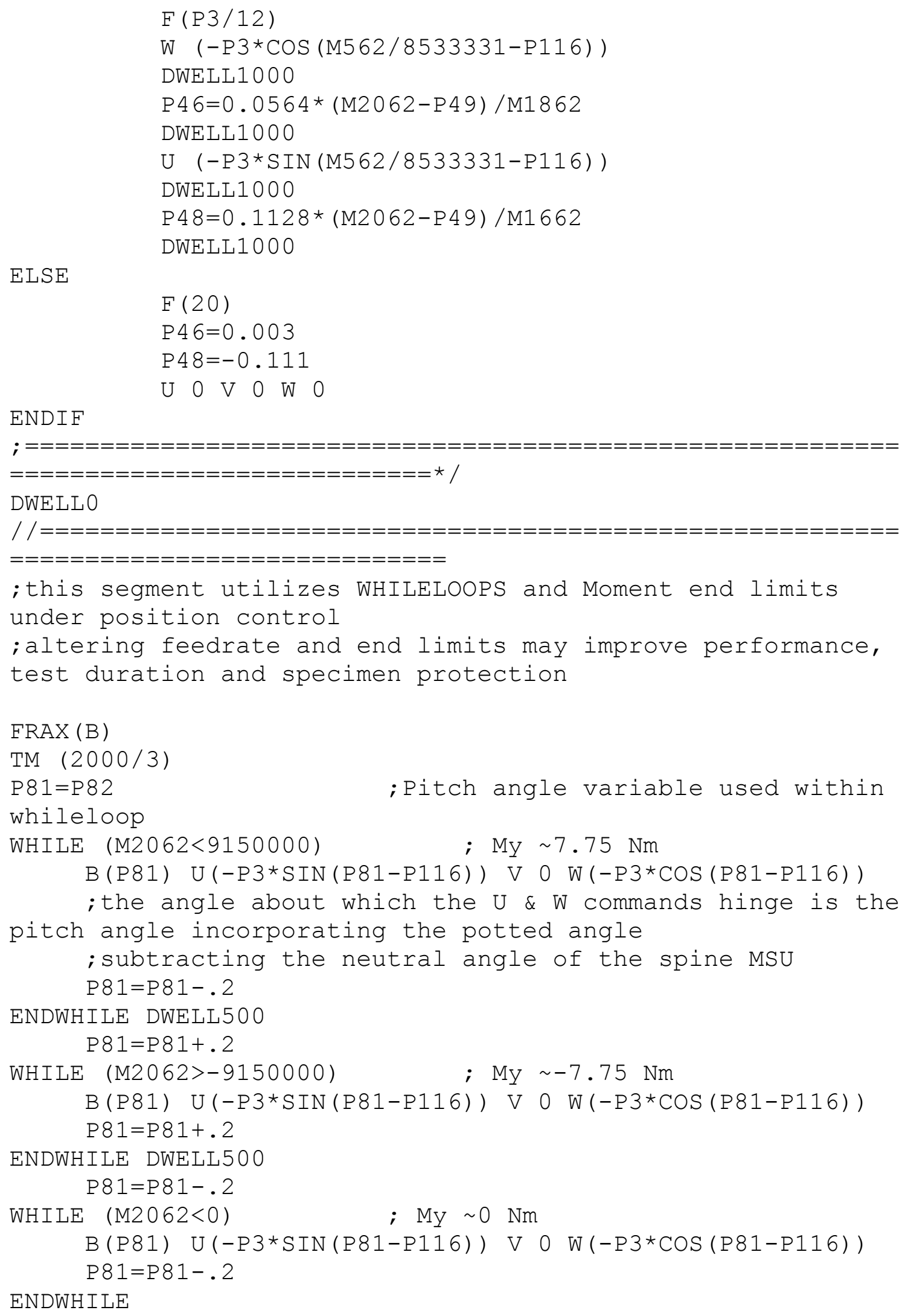




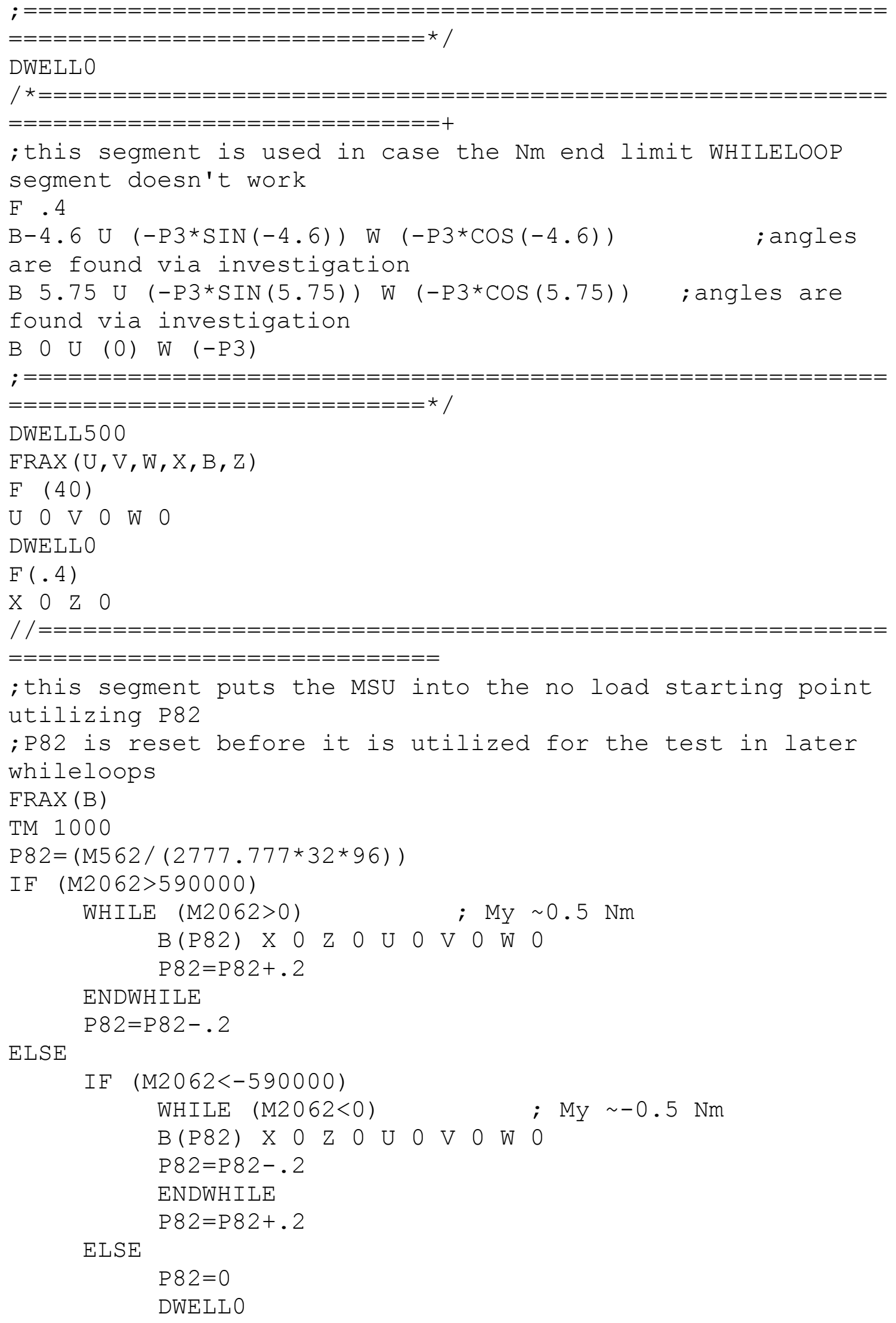




\section{ENDIF}

ENDIF

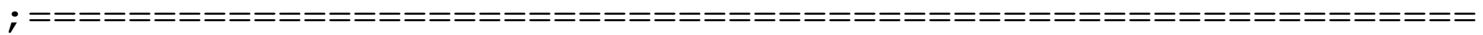

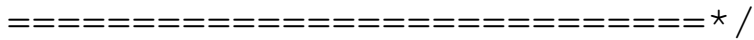

$\mathrm{P} 46=0$

$\mathrm{P} 47=0$

$\mathrm{P} 48=0$

CLOSE

\section{B.2 Program 15 Trunk Weight Pitch Position}

//THIS PROGRAM IS TO BE RUN AFTER

PLC14 CB Spine PitchPos ForceCtrl . pmc

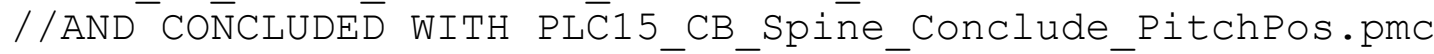

close; Close any buffer opened

delete gather; Erase unwanted gathered data

delete lookahead

; undefine all - Erase coordinate definitions in all

coordinate systems

; \&2DEFINE LOOKAHEAD 1500,20

OPEN PROG 15 CLEAR; Open buffer to be written

; DEFINE P80 as off-sagittal angle, convention: left turn is negative, right turn is positive

; oriented as clock placed atop head, clock face upwards

(clockwise sign orientation)

$\mathrm{P} 80=0 \quad$; off-sagittal angle, for only sagittal

bending, $\mathrm{P} 80=0$, unit: degrees

; DEFINE P3 as trunk weight force (units in Newtons)

$\mathrm{P} 3=400$

\&2 $\quad ;$ Force/Torque Coordinate system

$\operatorname{FRAX}(\mathrm{U}, \mathrm{V}, \mathrm{W}, \mathrm{X}, \mathrm{B}, \mathrm{Z})$

LIN; Linear interpolation

; INC; Incremental mode, moves are relative to last location ABS; Absolute mode, moves are relative to coordinate system grid

TA 450 ; Acceleration time in msec

TS 150 ; S-curve acceleration component in msec

\&2\%100 ; Feedrate Override value

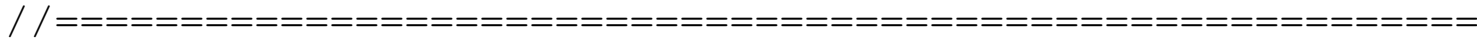

$=\mathrm{C}=\mathrm{=}=\mathrm{=}=\mathrm{=}=\mathrm{=}=\mathrm{=}=\mathrm{=}=\mathrm{=}=\mathrm{=}=\mathrm{=}=$ 
; this segment puts the MSU into the no load starting point utilizing $\mathrm{P} 82$

; P82 is reset before it is utilized for the test in later whileloops

$\operatorname{FRAX}(\mathrm{B})$

TM 1000

$\mathrm{P} 82=0$

IF $\quad(\mathrm{M} 2062>590000)$

WHILE $($ M2062>0) ; My $\sim 0.5 \mathrm{Nm}$

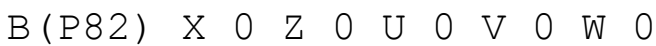

$\mathrm{P} 82=\mathrm{P} 82+.2$

ENDWHILE

$\mathrm{P} 82=\mathrm{P} 82-.2$

ELSE

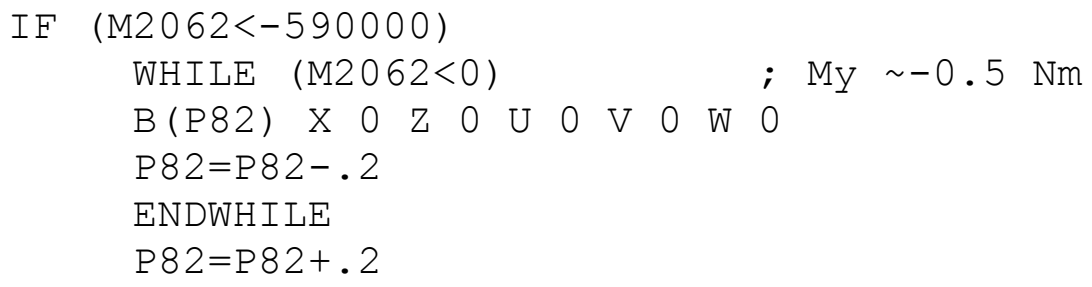

ELSE

$\mathrm{P} 82=(\mathrm{M} 562 /(2777.777 * 32 * 96))$

$\begin{array}{lllllll}\mathrm{U} & 0 & \mathrm{~V} & 0 & \mathrm{~W} & 0\end{array}$

$\mathrm{F} .4$

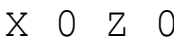

DWELLO

ENDIF

ENDIF

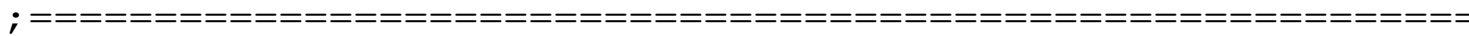

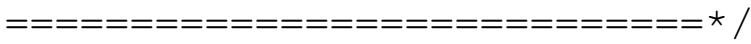

DWELLO

$/ / \mathrm{l}=\mathrm{C}=\mathrm{=}=\mathrm{=}=\mathrm{=}=\mathrm{=}=\mathrm{=}=\mathrm{=}=\mathrm{=}=\mathrm{=}=\mathrm{=}=\mathrm{=}=\mathrm{=}=\mathrm{=}=\mathrm{=}=\mathrm{=}=\mathrm{=}=\mathrm{=}=\mathrm{=}=\mathrm{=}=\mathrm{=}=\mathrm{=}$ $==\mathrm{=}=\mathrm{=}=\mathrm{=}=\mathrm{=}=\mathrm{=}=\mathrm{=}=\mathrm{=}=\mathrm{=}=\mathrm{=}=\mathrm{=}=$

; this segment conducts the force reference frame transform for single segment MSUs

; under the conditions that the program is operating under position control in some capacity

; (not full torque) AND that there is a desired applied

load. Be sure to check the commanded position

; control motor to ensure that the correct motor is being addressed (ex. B is the pitch motor)

IF $\quad(P 3 !=0)$

$\mathrm{P} 49=\mathrm{M} 2062$

$\mathrm{F}(\mathrm{P} 3 / 12)$

W $(-\mathrm{P} 3 * \operatorname{CoS}(\mathrm{M} 562 / 8533331+\mathrm{P} 119-\mathrm{P} 116))$

DWELL1000

$\mathrm{P} 46=0.0564 *(\mathrm{M} 2062-\mathrm{P} 49) / \mathrm{M} 1862$ 


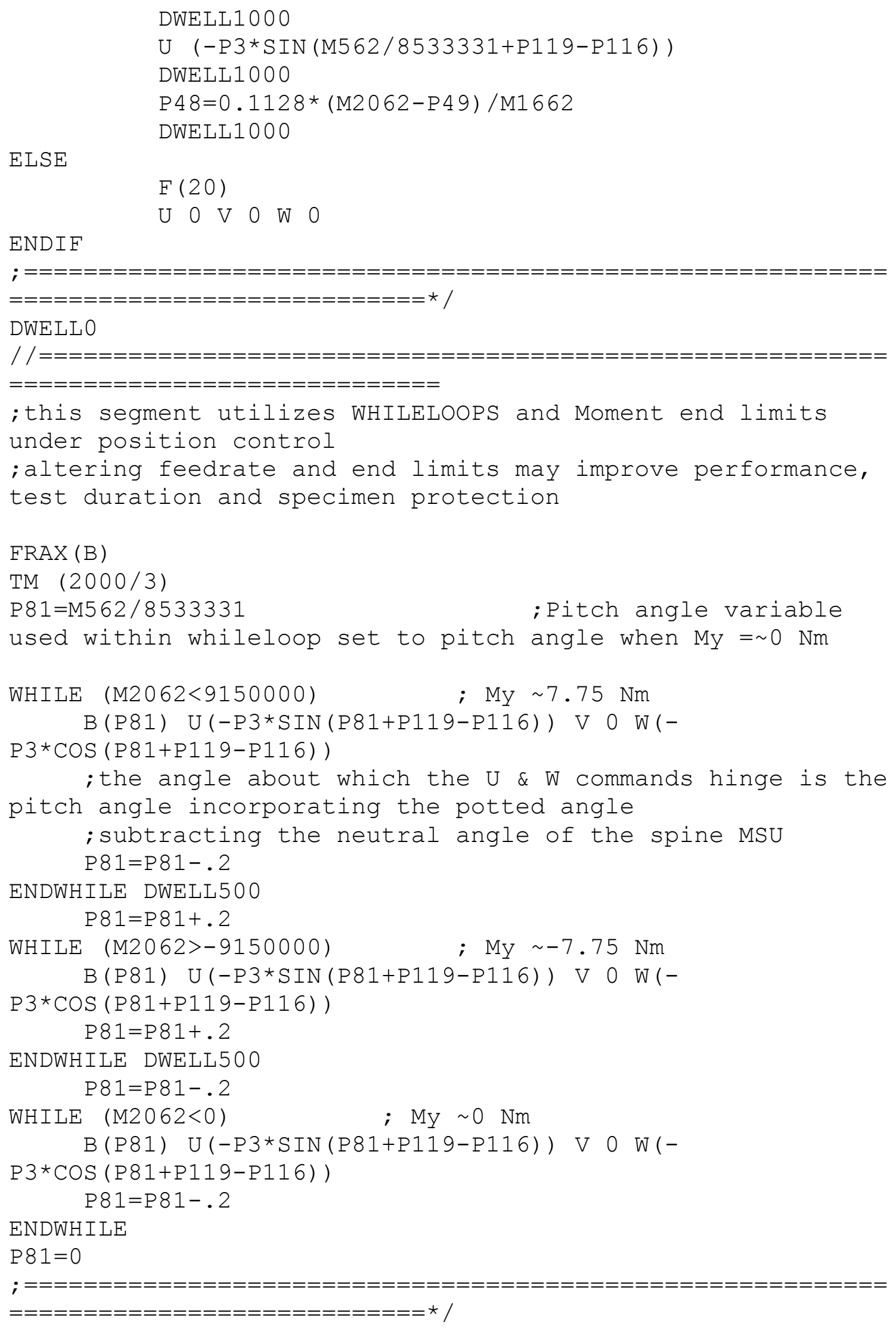




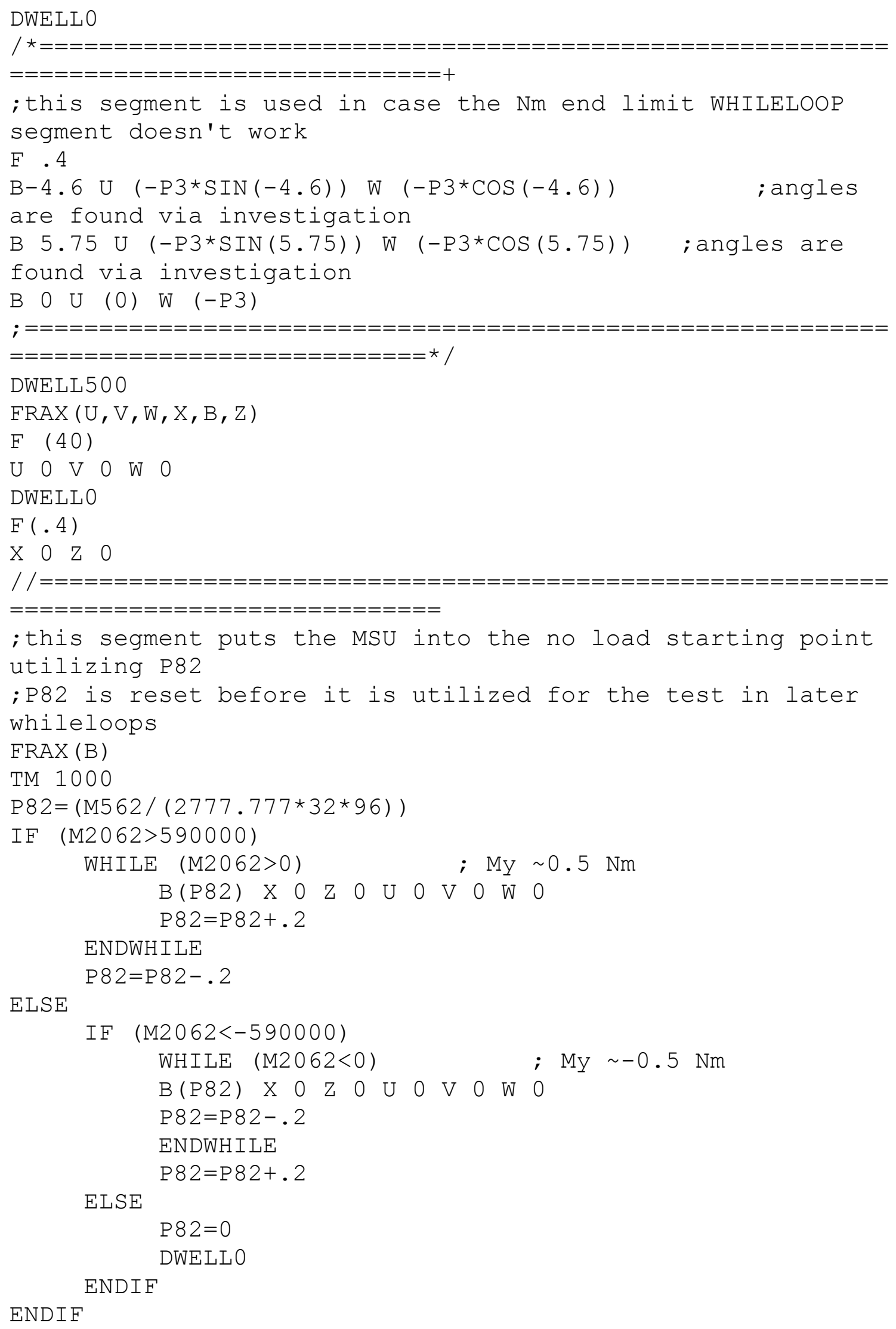




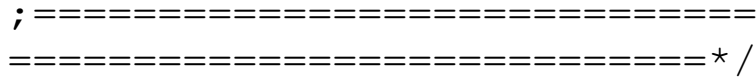

$P 46=0$

$\mathrm{P} 47=0$

$\mathrm{P} 48=0$

CLOSE

\section{B.3 Program 16 Follower Load Pitch Position}

//THIS PROGRAM IS TO BE RUN AFTER

PLC14_CB_Spine_ForceCtrl.pmc

//AND CON̄CLUDĒ WITH PLC15_CB_Spine_Conclude.pmc

close ; Close any buffer opened

delete gather ; Erase unwanted gathered data

delete lookahead

; undefine all - Erase coordinate definitions in all

coordinate systems

; \&1DEFINE LOOKAHEAD 1500,20

OPEN PROG 16 CLEAR ; Open buffer to be written

; DEFINE P80 as off-sagittal angle, convention: left turn is negative, right turn is positive

; oriented as clock placed atop head, clock face upwards

(clockwise sign orientation)

$\mathrm{P} 80=0 \quad$; off-sagittal angle, for only sagittal

bending, $\mathrm{P} 80=0$, unit: degrees

; DEFINE P3 as follower load force (units in Newtons)

$\mathrm{P} 3=400$

\&2 $\quad$; Force/Torque Coordinate system

$\operatorname{FRAX}(\mathrm{U}, \mathrm{V}, \mathrm{W}, \mathrm{X}, \mathrm{B}, \mathrm{Z})$

LIN; Linear interpolation

;INC; Incremental mode, moves are relative to last location ABS; Absolute mode, moves are relative to coordinate system grid

TA 450 ; Acceleration time in msec

TS 150 ; S-curve acceleration component in msec

\&2\%100 ; Feedrate Override value

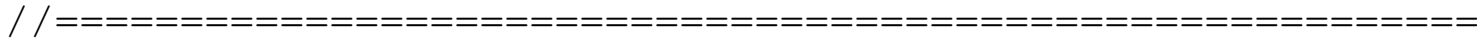

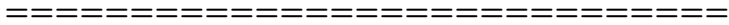

; this segment puts the MSU into the no load starting point utilizing P82 
;P82 is reset before it is utilized for the test in later whileloops

$\operatorname{FRAX}(\mathrm{B})$

TM 1000

$\mathrm{P} 82=0$

IF $(\mathrm{M} 2062>590000)$

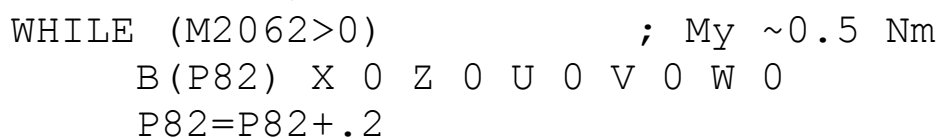

ENDWHILE

$\mathrm{P} 82=\mathrm{P} 82-.2$

ELSE

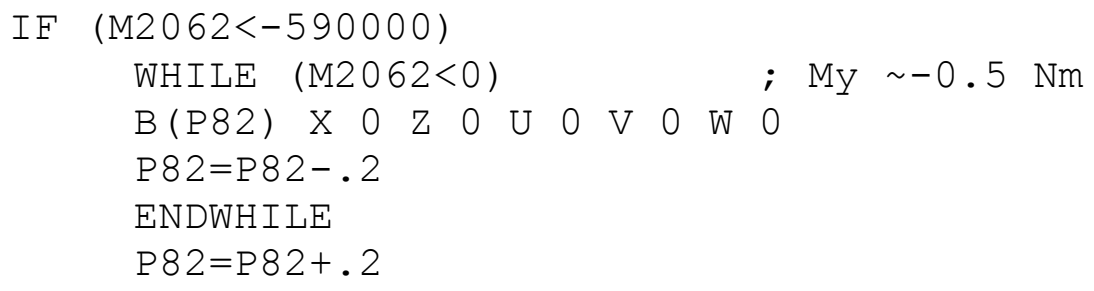

ELSE

$\mathrm{P} 82=(\mathrm{M} 562 /(2777.777 * 32 * 96))$

$\begin{array}{lllllllllll} & 0 & \mathrm{~V} & 0 & \mathrm{~W} & 0\end{array}$

$\mathrm{F} .4$

$\begin{array}{llllllll}X & 0 & Z & 0\end{array}$

DWELLO

ENDIF

\section{ENDIF}

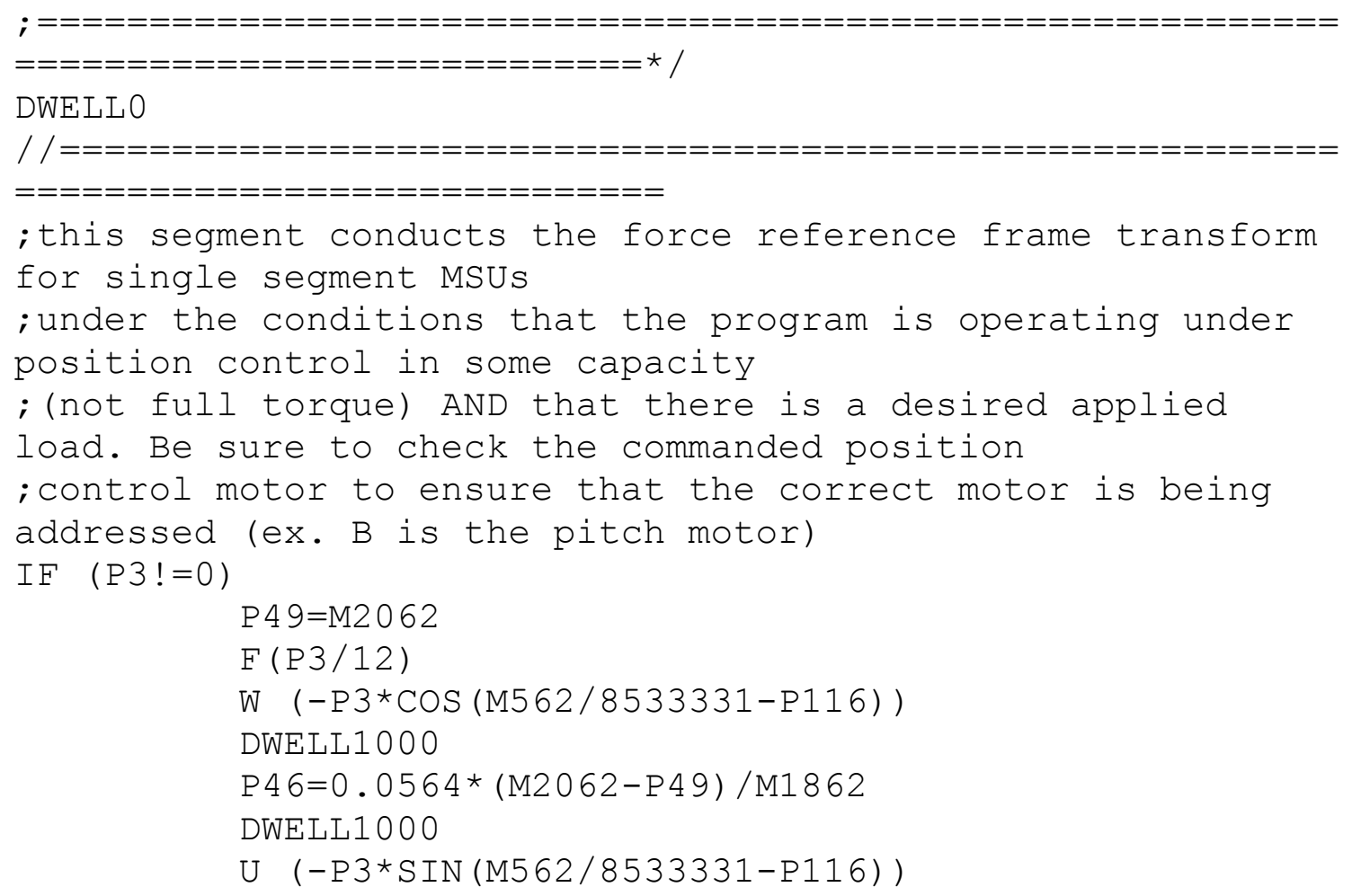


DWELL1000

$\mathrm{P} 48=0.1128 *(\mathrm{M} 2062-\mathrm{P} 49) / \mathrm{M} 1662$

DWELL1000

ELSE

F (20)

$\begin{array}{llllllllllllllll} & 0 & \mathrm{~V} & 0 & \mathrm{~W} & 0\end{array}$

ENDIF

; $=\mathrm{C}=\mathrm{=}=\mathrm{=}=\mathrm{=}=\mathrm{=}=\mathrm{=}=\mathrm{=}=\mathrm{=}=\mathrm{=}=\mathrm{=}=\mathrm{=}=\mathrm{=}=\mathrm{=}=\mathrm{=}=\mathrm{=}=\mathrm{=}=\mathrm{=}=\mathrm{=}=\mathrm{=}=\mathrm{=}=\mathrm{=}=\mathrm{=}$

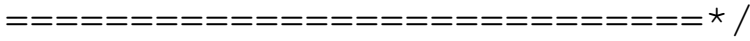

DWELLO

/ $/ \mathrm{C=}=\mathrm{=}=\mathrm{=}=\mathrm{=}=\mathrm{=}=\mathrm{=}=\mathrm{=}=\mathrm{=}=\mathrm{=}=\mathrm{=}=\mathrm{=}=\mathrm{=}=\mathrm{=}=\mathrm{=}=\mathrm{=}=\mathrm{=}=\mathrm{=}=\mathrm{=}=\mathrm{=}=\mathrm{=}=\mathrm{=}=\mathrm{=}$ $=\mathrm{E}=\mathrm{=}=\mathrm{=}=\mathrm{=}=\mathrm{=}=\mathrm{=}=\mathrm{=}=\mathrm{=}=\mathrm{=}=\mathrm{=}=$

; this segment utilizes WHILELOOPS and Moment end limits under position control

; altering feedrate and end limits may improve performance, test duration and specimen protection

$\operatorname{FRAX}(\mathrm{B})$

$\operatorname{TM}(2000 / 3)$

$\mathrm{P} 81=\mathrm{P} 82$

; Pitch angle variable used within

whileloop

WHILE $($ M2062<9150000) ; My $~ 7.75 \mathrm{Nm}$

$\mathrm{B}(\mathrm{P} 81) \quad \mathrm{U}(-\mathrm{P} 3 * \mathrm{SIN}(\mathrm{P} 81 / 2-\mathrm{P} 116)) \quad \mathrm{V} \quad 0 \quad \mathrm{~W}(-\mathrm{P} 3 * \mathrm{COS}(\mathrm{P} 81 / 2-$

$\mathrm{P} 116))$

; the angle about which the $U$ \& $W$ commands hinge is the pitch angle incorporating the potted angle

; subtracting the neutral angle of the spine MSU

$\mathrm{P} 81=\mathrm{P} 81-.2$

ENDWHILE DWELL500

$\mathrm{P} 81=\mathrm{P} 81+.2$

WHILE (M2062>-9150000) ; My -7.75 Nm

$\mathrm{B}(\mathrm{P} 81) \quad \mathrm{U}(-\mathrm{P} 3 * \mathrm{SIN}(\mathrm{P} 81 / 2-\mathrm{P} 116)) \quad \mathrm{V} 0 \mathrm{~W}(-\mathrm{P} 3 * \mathrm{COS}(\mathrm{P} 81 / 2-$

$\mathrm{P} 116))$

$\mathrm{P} 81=\mathrm{P} 81+.2$

ENDWHILE DWELL500

$\mathrm{P} 81=\mathrm{P} 81-.2$

WHILE $($ M2062<0) ; My $\sim 0 \mathrm{Nm}$

$\mathrm{B}(\mathrm{P} 81) \quad \mathrm{U}(-\mathrm{P} 3 * \mathrm{SIN}(\mathrm{P} 81 / 2-\mathrm{P} 116)) \quad \mathrm{V} \quad 0 \quad \mathrm{~W}\left(-\mathrm{P} 3{ }^{*} \mathrm{COS}(\mathrm{P} 81 / 2-\right.$

$\mathrm{P} 116))$

$\mathrm{P} 81=\mathrm{P} 81-.2$

ENDWHILE

$\mathrm{P} 81=0$

; $=\mathrm{=}=\mathrm{=}=\mathrm{=}=\mathrm{=}=\mathrm{=}=\mathrm{=}=\mathrm{=}=\mathrm{=}=\mathrm{=}=\mathrm{=}=\mathrm{=}=\mathrm{=}=\mathrm{=}=\mathrm{=}=\mathrm{=}=\mathrm{=}=\mathrm{=}=\mathrm{=}=\mathrm{=}=\mathrm{=}=\mathrm{=}=\mathrm{=}$

$==\mathrm{=}=\mathrm{=}=\mathrm{=}=\mathrm{=}=\mathrm{=}=\mathrm{=}=\mathrm{=}=\mathrm{=}=\mathrm{=}=\mathrm{=} /$

DWELLO

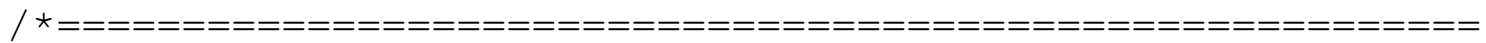

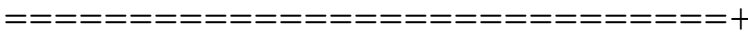


; this segment is used in case the $\mathrm{Nm}$ end limit WHILELOOP segment doesn't work

F. . 4

$\mathrm{B}-4.6 \mathrm{U}(-\mathrm{P} 3 * \operatorname{SIN}(-4.6)) \mathrm{W}(-\mathrm{P} 3 * \operatorname{COS}(-4.6)) \quad$; angles

are found via investigation

B $5.75 \mathrm{U}(-\mathrm{P} 3 * \operatorname{SIN}(5.75)) \mathrm{W}(-\mathrm{P} 3 * \operatorname{COS}(5.75))$; angles are found via investigation

B 0 O

; $=\mathrm{=}=\mathrm{=}=\mathrm{=}=\mathrm{=}=\mathrm{=}=\mathrm{=}=\mathrm{=}=\mathrm{=}=\mathrm{=}=\mathrm{=}=\mathrm{=}=\mathrm{=}=\mathrm{=}=\mathrm{=}=\mathrm{=}=\mathrm{=}=\mathrm{=}=\mathrm{=}=\mathrm{=}=\mathrm{=}=\mathrm{=}$

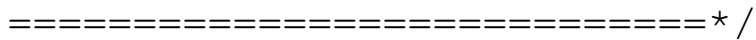

DWELL500

$\operatorname{FRAX}(U, V, W, X, B, Z)$

F $(40)$

$\begin{array}{lllllllllllll}U & 0 & V & 0 & W & 0\end{array}$

DWELLO

$\mathrm{F}(.4)$

$\mathrm{X} \quad \mathrm{O} \quad \mathrm{Z} \quad \mathrm{O}$

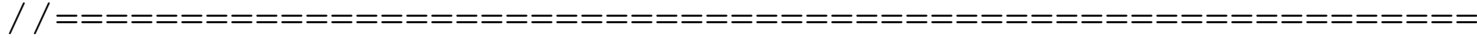

$=\mathrm{C}=\mathrm{=}=\mathrm{=}=\mathrm{=}=\mathrm{=}=\mathrm{=}=\mathrm{=}=\mathrm{=}=\mathrm{=}=\mathrm{=}=\mathrm{=}=\mathrm{=}$

; this segment puts the MSU into the no load starting point utilizing $\mathrm{P} 82$

;P82 is reset before it is utilized for the test in later whileloops

$\operatorname{FRAX}(\mathrm{B})$

TM 1000

$\mathrm{P} 82=(\mathrm{M} 562 /(2777.777 * 32 * 96))$

IF $(\mathrm{M} 2062>590000)$

WHILE $($ M2062>0) ; MY $\sim 0.5 \mathrm{Nm}$

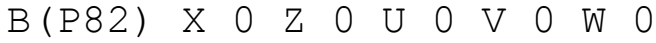

$\mathrm{P} 82=\mathrm{P} 82+.2$

ENDWHILE

$\mathrm{P} 82=\mathrm{P} 82-.2$

ELSE

IF $\quad(\mathrm{M} 2062<-590000)$

WHILE $($ M2062<0 $\quad ; \mathrm{My} \sim-0.5 \mathrm{Nm}$

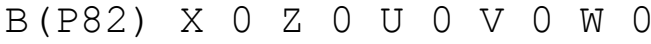

$\mathrm{P} 82=\mathrm{P} 82-.2$

ENDWHILE

$\mathrm{P} 82=\mathrm{P} 82+.2$

ELSE

$P 82=0$

DWELLO

ENDIF

ENDIF

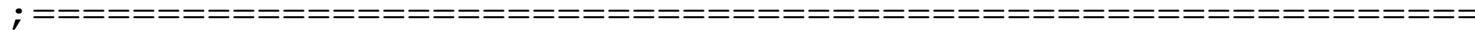

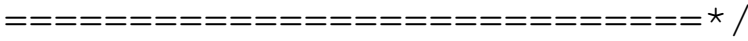

$P 46=0$ 
P $47=0$

$\mathrm{P} 48=0$

CLOSE 


\section{VITA}

Charles Ramsey Bennett was born in 1988 to Gregory Ray and Mary Sark Bennett in Atlanta, Georgia. He has a sister four years the younger named Emily Ann Bennett. After graduating from Chamblee Charter High School of the DeKalb County Public School District he attended his parents' alma mater, Clemson University in Clemson, South Carolina. He graduated from the Calhoun Honors College at Clemson University summa cum laude with a Bachelor of Science degree in bioengineering with emphasis in biomaterials. Charles then pursued a Master of Science degree in biomedical engineering and imaging in the Joint Program from the University of Tennessee and the University of Memphis at the University of Tennessee Health Science Center in Memphis, Tennessee. His area of focus was in vitro spinal biomechanics with special interest in robotics, control theory, and RTLC as they apply to the development of a novel biomechanical testing apparatus. He married Rachel Elizabeth Bennett in June 2011. 